\title{
WestVirginiaUniversity
}

THE RESEARCH REPOSITORY @ WVU

Graduate Theses, Dissertations, and Problem Reports

2020

\section{Ultrafast Optical Properties of La0.7Sr0.3MnO3 Thin Films}

\author{
Saeed Yousefi Sarraf \\ sayousefisarraf@mix.wvu.edu
}

Follow this and additional works at: https://researchrepository.wvu.edu/etd

Part of the Condensed Matter Physics Commons, and the Optics Commons

\section{Recommended Citation}

Yousefi Sarraf, Saeed, "Ultrafast Optical Properties of La0.7Sr0.3MnO3 Thin Films" (2020). Graduate Theses, Dissertations, and Problem Reports. 7607.

https://researchrepository.wvu.edu/etd/7607

This Dissertation is protected by copyright and/or related rights. It has been brought to you by the The Research Repository @ WVU with permission from the rights-holder(s). You are free to use this Dissertation in any way that is permitted by the copyright and related rights legislation that applies to your use. For other uses you must obtain permission from the rights-holder(s) directly, unless additional rights are indicated by a Creative Commons license in the record and/ or on the work itself. This Dissertation has been accepted for inclusion in WVU Graduate Theses, Dissertations, and Problem Reports collection by an authorized administrator of The Research Repository @ WVU.

For more information, please contact researchrepository@mail.wvu.edu. 
Graduate Theses, Dissertations, and Problem Reports

2020

Ultrafast Optical Properties of La0.7Sr0.3MnO3 Thin Films

Saeed Yousefi Sarraf

Follow this and additional works at: https://researchrepository.wvu.edu/etd

Part of the Condensed Matter Physics Commons, and the Optics Commons 


\title{
Ultrafast Optical Properties of $\mathrm{La}_{0.7} \mathrm{Sr}_{03} \mathrm{MnO}_{3}$ Thin Films
}

\section{Saeed Yousefi Sarraf}

\author{
Dissertation submitted to the Eberly \\ College of Arts and Sciences at West \\ Virginia University in partial fulfillment \\ of the requirements for the degree of
}

\section{Doctor of Philosophy \\ in}

Physics

\author{
Mikel Holcomb, Ph.D., Chair \\ Alan Bristow, Ph.D. \\ Aldo Romero, Ph.D. \\ Matthew Johnson, Ph.D. \\ Fabien Goulay, Ph.D. \\ Department of Physics and Astronomy \\ Morgantown, West Virginia \\ 2020
}

Keywords: complex oxides, perovskites oxides, ultrafast spectroscopy, transient reflectivity, spectroscopic ellipsometry, free carrier dynamics, spin dynamics, transient strain waves, sound velocity, wavelet analysis, morse wavelet

Copyright 2020, Saeed Yousefi Sarraf 


\section{Abstract \\ Ultrafast Optical Properties of Lao.7Sro.3MnO3 Thin Films}

\section{Saeed Yousefi Sarraf}

Thin film solids often exhibit different physical properties in the ultra-thin regime. Enhancement of surface to bulk ratio results in the domination of surface/interface related phenomena such as surface recombination. Moreover, in the ultra-thin regime, quantum size and quantum confinement effects can alter the band gap of the system and constrain the strain wave propagation in the thin film. Ultrafast properties of solids can also be drastically altered in the ultra-thin regime due to the aforementioned phenomena. Experimentally, observation of these phenomena is challenging due to the insufficient material to absorb and interact with the electromagnetic wave.

This dissertation addresses the altercation of the ultrafast phenomena in the ultra-thin regime in complex oxide $\mathrm{La}_{0.7} \mathrm{Sr}_{0.3} \mathrm{MnO}_{3}$ (LSMO) films. To accomplish this goal, a degenerate optical ultrafast pumpprobe setup was developed to perform transient reflectivity (TR) experiments in cross linear and circular polarization configurations. Analyzing methods were ranged from multi-decay component modeling to understand the ultrafast dynamics, to the newly introduced wavelet analysis to study the propagation of transient strain waves in ultra-thin films. Moreover, density functional theory has been used as a complementary method to further prove the claims made based on the observations.

Spectroscopic ellipsometry of LSMO ultra-thin films illustrate optical transitions between the substrate $\left(\mathrm{STO}_{3}\right)$ and thin film's orbitals, at the interface. It has been indicated that, in the ultra-thin regime, the dominated surface recombination results in a complete excited carriers' energy loss in less than a few hundreds of picoseconds. Hence, the surface recombination, that is known to be detrimental for device purposes, is introduced as a phenomenon that can be useful for ultrafast switches in ultra-thin films. Moreover, the ultrafast photoinduced spin polarization of LSMO thin films exhibit a sharp decrease in ultrafast regime ( $1 \mathrm{ps}$ ) which suggest LSMO thin films to be used in ultrafast magnetic switches. Wavelet analysis was introduced as an efficient method, compared to the Fourier transform, to analyze oscillatory modes superimposed on the TR signal which are caused by the propagation of strain wave longitudinally in the thin film. As a result, it has been illustrated that the sound velocity in LSMO ultra-thin films increases by decreasing the film thickness. Strong energy transfer between the thin film and the substrate has also been observed using wavelet analysis. 


\begin{abstract}
Acknowledgment
There are many thanks to be given to the people who helped me to complete this thesis. First, I would like to express my sincere thanks to my advisor Dr. Holcomb. As a supervisor, she showed me the path, guided through it, and provided necessary needs for me to follow it. Her patience during the many fruitless months of research encouraged me to try harder to achieve the result. Many thanks to Dr. Bristow for his knowledge and advice. He was a help whenever I needed and without his help, this thesis could not be finished. I would also like to thank Dr. Romero and his students for their collaboration and providing more insights into the scientific problems. Last but not least, I thank many people in Dr. Holcomb's group who helped me to develop a variety of materials for this thesis.

On a more personal note, I would like to express how much gratitude I owe my family and friends for being beside me throughout my Ph.D. The fact that I had my loving mother, thoughtful father and kind sister, back home caring about my success was the main motive for me to tolerate the difficulties throughout these years. Their support, despite knowing that they will not be able to see me for many years, is still unbelievable to me. I would also like to thank many friends here in the U.S. who made the years far from the family and home bearable.
\end{abstract}

Saeed Yousefi

April 2020 


\section{Table of content}

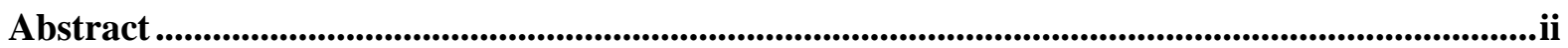

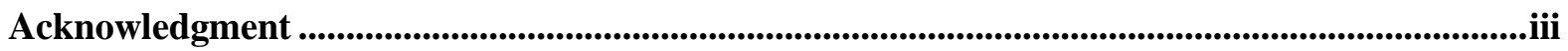

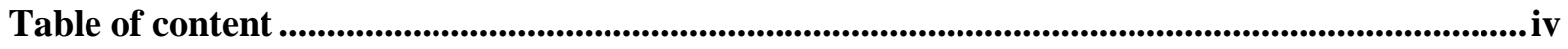

Chapter 1. Introduction ......................................................................................................................................1

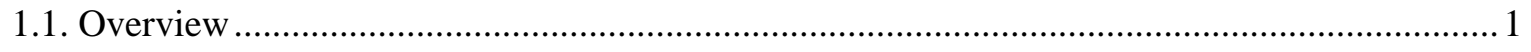

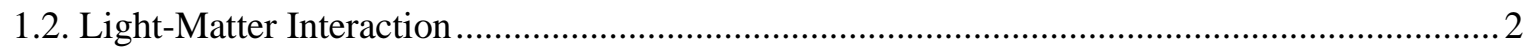

1.3. Ultrafast Spectroscopy and Ultrafast Phenomena in Solids ............................................... 4

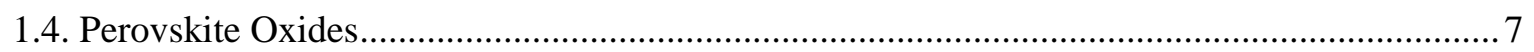

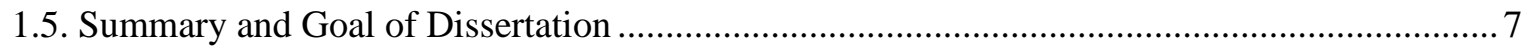

Chapter 2. Sample Preparation and Characterization ................................................................................10

2.1. Perovskite Oxide $\mathrm{La}_{0.7} \mathrm{Sr}_{0.3} \mathrm{MnO}_{3}$ This Films.................................................................. 10

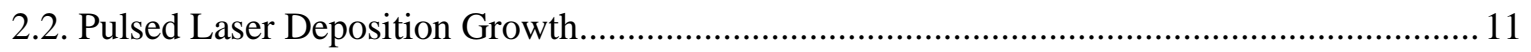

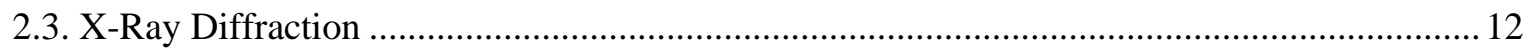

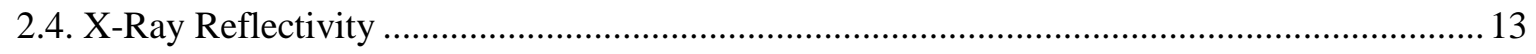

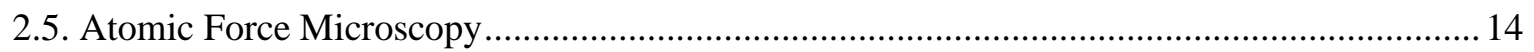

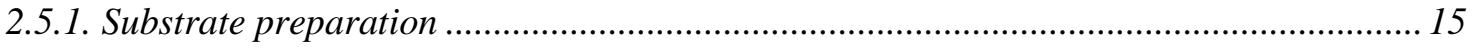

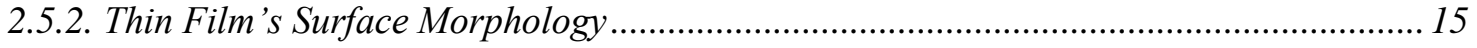

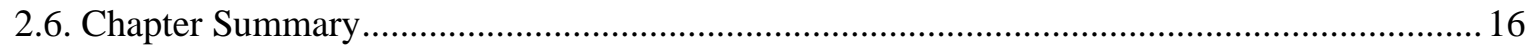

Chapter 3. Static Optical Properties.............................................................................................................17

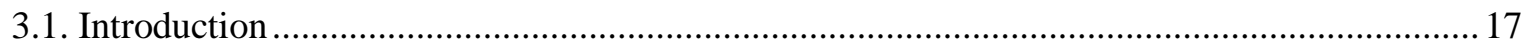

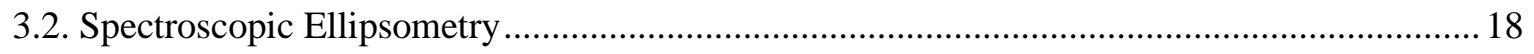

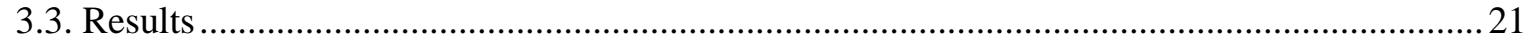

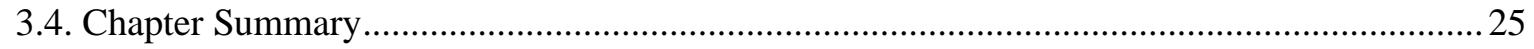

Chapter 4. Transient Reflectivity .............................................................................................................2

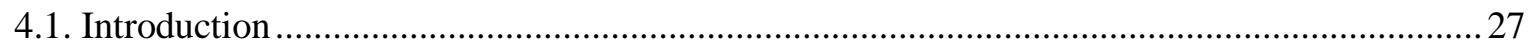

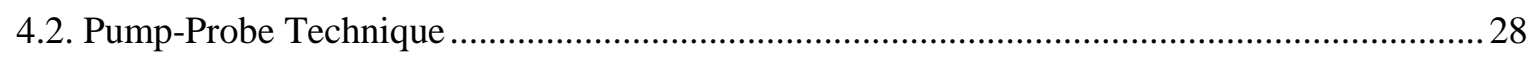




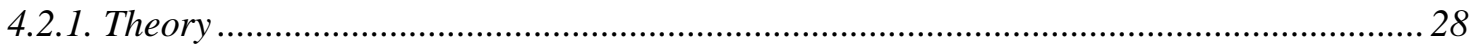

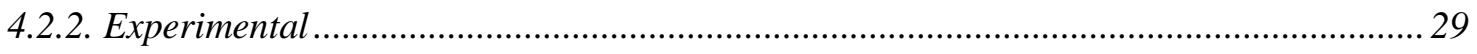

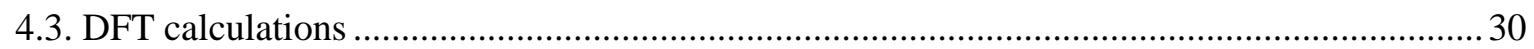

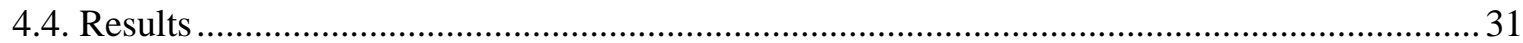

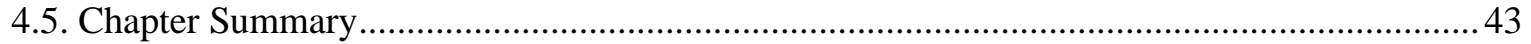

Chapter 5. Coherent Acoustic Phonons.....................................................................................................45

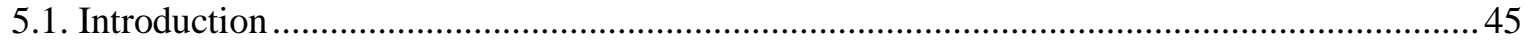

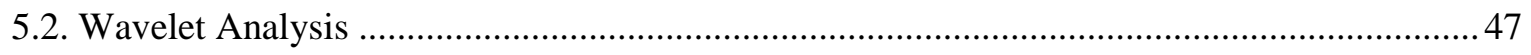

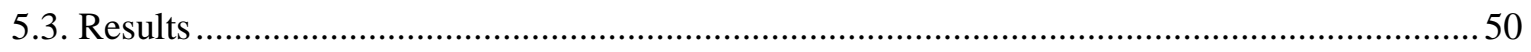

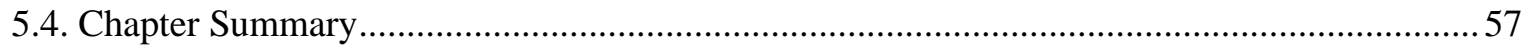

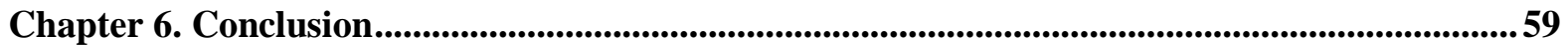

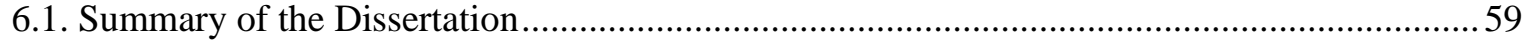

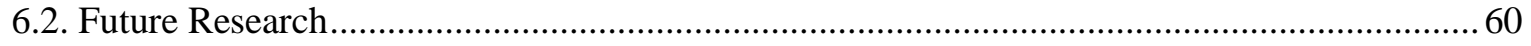

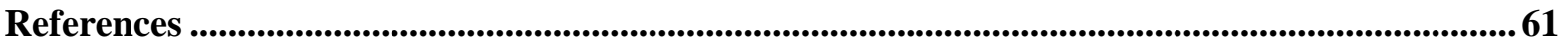




\section{Chapter 1. Introduction}

\subsection{Overview}

With thin film technology reaching its size limits, challenges have emerged to understand the physical properties of solids in ultra-thin regime. By decreasing the film thickness various phenomena such as quantum confinement and quantum size effect drastically change the properties of solids. For example, for thicknesses of less than $\sim 10 \mathrm{~nm}$, by squeezing electrons and holes down to exciton Bohr radius, quantum confinement results in bandgap increase. Moreover, as the thickness decreases and surface to bulk ratio increases, surface phenomena such as surface recombination dominate the bulk features. Particularly, understanding the light and matter interactions which inform about various quasiparticle interactions such as carrier-carrier scattering, carrier-phonon scattering, spin-lattice scattering, acoustic and optical phonon propagation, spin wave propagation, heat propagation, transient strain wave propagation, etc. in ultra-thin regime can help the industry to increase the efficiency of devices as they decrease the cost by using less material. For example, devices such as solar cells and ultrafast switches are crucially dependent on information regarding ultrafast carrier dynamics. While solar cells are in favor of slower recombination mechanisms, in ultrafast switches faster recombination mechanisms mean faster switches. Information regarding transient strain waves can be used in ultrasonic sensors, where the subject material is sensitive to the electromagnetic waves, such as biological tissues. Moreover, understanding the spin related interactions are necessary for spintronic devices such as hard disk drives, magnetic RAMs, magnetic tunnel junctions, etc.

A promising family of materials known as strongly correlated complex oxides has received considerable attention due to their applications in fuel cells, transducers, actuators, batteries, magnetic storage devices and many more. The strong correlation between d-orbital's electrons in these materials results in drastic changes in physical properties by changing a variety of parameters such as thickness. Among strongly correlated complex oxides, $\mathrm{La}_{0.7} \mathrm{Sr}_{0.3} \mathrm{MnO}_{3}$ is a colossal magnetoresistant that has attracted attention in the past few decades and has been used in solid oxide fuel cells and magnetic tunnel junctions.

Many optical experiments have been done on LSMO thin films on various conditions to understand the light-matter interaction in these complex oxides. In 1999 Nomerovannaya et al ${ }^{1}$ reported on fundamental changes in the optical spectra of $\mathrm{La}_{1-\mathrm{x}} \mathrm{Sr}_{\mathrm{x}} \mathrm{MnO}_{3}$ by changing $\mathrm{Sr}$ doping level, using a spectroscopic ellipsometer. Liu et $_{a l} \mathrm{l}^{2}$ (2005) measured room temperature reflectance and transmittance spectra of LSMO thin films with various thicknesses of $400 \mathrm{~nm}, 100 \mathrm{~nm}$ and 10 
$\mathrm{nm}$, and reported on the dominance of the substrate features in the optical spectra of LSMO with the thickness of less than $10 \mathrm{~nm}$. Martino et $a l^{3}$ (2011) and Cesaria et al ${ }^{4}$ (2013) performed UVVis-NIR spectroscopy on $200 \mathrm{~nm}$ LSMO films with various oxygen vacancies and reported on different optical transitions that are dependent on the oxygen vacancies. In 2000 Lobad et al ${ }^{5}$ reported on spin-lattice and electron-phonon relaxations for a $75 \mathrm{~nm}$ LSMO film using a temperature dependence ultrafast spectroscopy and two-temperature models. Parlato et $a l^{6}$ (2013) performed temperature dependence ultrafast spectroscopy on $100 \mathrm{~nm}$ LSMO film and analyzed the result using two-temperature models. They reported on electron-phonon relaxation, followed by spin-lattice relaxation and heat diffusion. Moreover, they reported on a very low signal to noise ratio at temperatures above $130 \mathrm{~K}$. Using time-resolved magneto optical Kerr effect, in 2009 Muller et $a l^{7}$ reported on demagnetization time of 400-600 ps for LSMO thin film. Ren et $a l^{8}$ in 2007 focused on coherent longitudinal acoustic phonons, using ultrafast spectroscopy, in LSMO thin films with thicknesses of $200 \mathrm{~nm}, 300 \mathrm{~nm}$ and $400 \mathrm{~nm}$, and reported on sound velocity of $\sim 6500$ $\mathrm{m} / \mathrm{s}$ for LSMO thin films.

However, the main challenge rising in optical experiments as the film thickness reaches the ultra-tin regime, is the fact that by decreasing the film thickness insufficient amount of material exists to absorb the electromagnetic wave and interact with it. Eventually, in ultra-thin regime this problem results in a drastic hindrance in the optical experiments. Therefore, while understanding the light-matter interaction in ultra-thin regime can help the industry to increase the efficiency and reduce the cost of devices, not enough information has been provided in the ultra-thin regime, yet. In this dissertation, we tackle this challenge that is facing the optical measurements, ranging from static optical properties to ultrafast dynamics and elastic properties, in ultra-thin complex oxide LSMO films.

\subsection{Light-Matter Interaction}

Light-matter interactions have been one of the most interesting topics in physics for a long time, which results in various physical properties. Prior to quantum mechanics, maybe the most well-known light-matter interaction that had excited scientists was the reasoning behind the fact that we see colors. By the efforts of Lorentz, we now know the physics behind colors is based on how the matter absorb, reflect and transmit the light. These phenomena can be theoretically described using the Lorentz oscillator model, which is a classical model. In this model, the electron is approximated to be bound to the nucleus using a spring-like force. The bound electron that is

affected by the nucleus has an effective mass. Hence this electron with new effective mass is called electron quasiparticle. The interaction of light and a quasiparticle is responsible to create colors. ${ }^{9}$ 


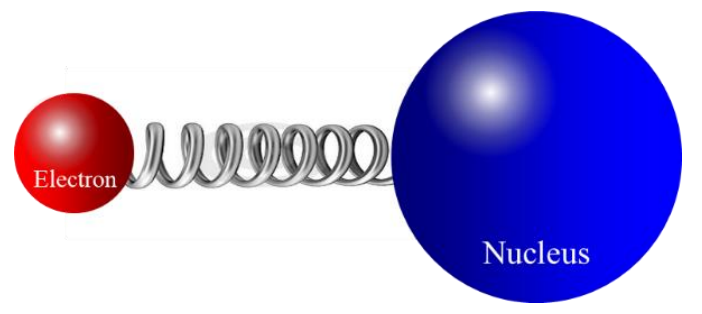

Figure 1. Lorentz model's representation of electron quasiparticle.

The complication with the classical Lorentz oscillatory model is that real materials are a collection of Lorentz oscillators with different frequencies. Quantum mechanics in which the fundamental particles have the properties of both particles and waves simultaneously, describes the distribution of the frequencies into bands via the wave formalism. The wave representation of particles is the core base of dispersion of oscillatory model, quasiparticles, and their interactions. Quasiparticles are not limited to the electron quasiparticle. Multiple quasiparticles have been discovered such as holes, phonons, plasmons, magnons, excitons, etc.

Each of these quasiparticles can interact with light, and create a complex system of interactive particles, understanding which can help to improve devices' efficiency and create new devices. Specifically, modern optoelectronic devices that can generate, detect and manipulate the light, are feasible using light-matter interactions. Lasers, ultrafast optical switches, ultrafast magnetic switches, optical waveguides for different light modes that can enhance and control the propagation of light, light emitters and energy harvesters are some of the most important devices made based on the concept of light-matter interactions. ${ }^{9}$

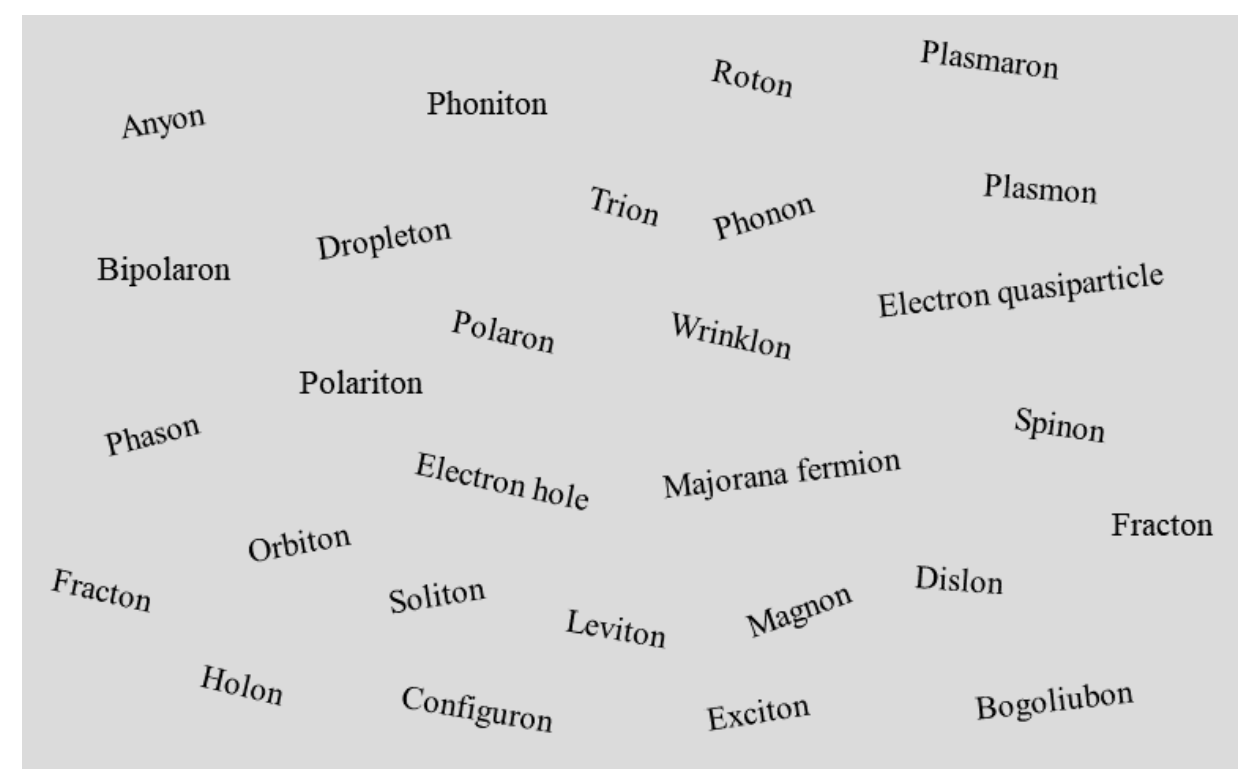

Figure 2. Various quasiparticles. 
Another aspect of quasiparticles is their interaction with each other that, in some cases, can be detrimental for devices. However, if understood well, these interactions can be used to control new devices. Electron-phonon interaction, phonon-phonon interaction, electron-electron interaction, electron-photon interaction, phonon-photon interaction and photon-photon interaction are some of the most common quasiparticle interactions in the solids. Moreover, in defective solids (all solids), defects and dislocations can interact. However, since they have much higher effective masses than the other quasiparticles, they count as immobile. Recombination centers at surfaces and interfaces can also be counted as defects. Surface and interface states, due to breaking in the bonds of the solid, can also interact with the ones from the bulk. These states can result in loss of excited carriers by recombination which eventually will result in efficiency reduction of photonic devices such as solar cells, lasers and detectors. Moreover, surface-specific quasiparticles such as surface plasmons and their interaction with an electromagnetic wave have practical effects on the functionality of the device. ${ }^{10}$ Hence, understanding nanoscale light-matter interactions, where the surface effects become nonnegligible, are crucial new photonic devices. ${ }^{11}$

Time evolution and the dynamics of the solids are defined based on these quasiparticle interactions. Quasiparticles' dynamics can be approximated using, in the simplest form, a relaxation time, phenomenologically. In this modulization, average scattered time for each type of the quasiparticle defines the exact lifetime of that interaction. And hence, the time evolution of the solid can be modeled. To develop new ultrafast devices and improve the efficiency of the current ones, a full understanding of the light-matter interaction, quasiparticle interactions, and their life is necessary. ${ }^{12}$

\subsection{Ultrafast Spectroscopy and Ultrafast Phenomena in Solids}

Quasiparticle interactions in solids happen on an ultrafast time scale. Competition between the lattice, charge, spin and orbitals, and considering their scattering time, creates a complex dynamic in the solid. Most of these competitions can be understood using the band structure of the solid. An occupied valance band (possibly) separated apart from the empty conduction bands construct the band structure of the system. Any excitation from the equilibrium in the energy of the system will recombine back to the equilibrium energy states, in a time scale that defines the dynamic of the system. Hence, any quasiparticle interaction that can manipulate the band structure of the system, will have some effects on the dynamics of the system. ${ }^{12}$ The Coulomb interaction between electrons and holes can create hydrogenic series of exciton bound states which have a drastic influence on the band structure of the system. Many studies have been performed on the dynamics of excitons in solids. ${ }^{13}$ 
Periodicity of the lattice results in quantized vibrations of the lattice known as phonons. Depending on the normal modes of the vibrations, optical and acoustic phonons will, generally speaking, affect the energy levels of the solid. Moreover, the lifetime of the scattering processes in solids due to interactions between quasiparticles such as electrons, holes, excitons and phonons with each other, lattice and defects, plays an important role in ultrafast dynamics of the solids. Interaction of carriers and phonons plays an important role in the conversion between momentum and energy in the system, transferring between lattice and carriers. Hence the relaxation and dynamics of the excited carriers can be drastically affected by carrier phonon interaction. ${ }^{14}$

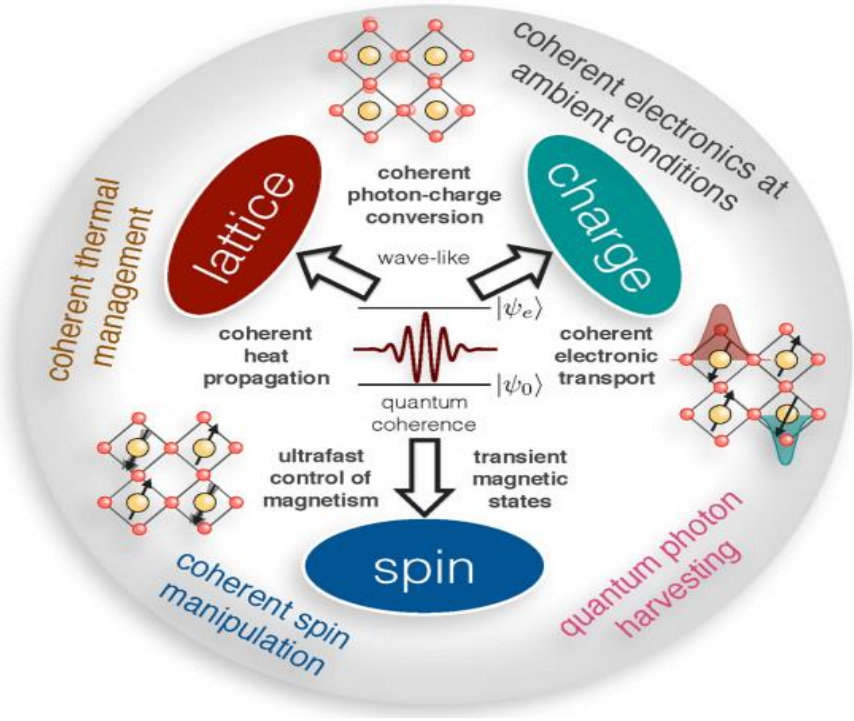

Figure 3. The ultrafast phenomenon in solids, taken from Gandolfi et. al. ${ }^{15}$

Carriers can also get scattered by other carriers. This scattering phenomenon determines the energy transfer between carriers and results in the thermalization of non-equilibrium non-thermal carries. Depending on the system, electron-electron, hole-hole and electron-hole scatterings can change the dynamics of the system. Spin relaxation processes such as magnetization precession (1 ps $-1 \mathrm{~ns})$, spin-orbit interaction ( $0.1 \mathrm{ps}-1 \mathrm{ps})$, exchange interaction ( $0.01 \mathrm{ps}-0.1 \mathrm{ps})$, spin waves and single spin relaxation ( $1 \mathrm{fs}$ ) can also drastically change the dynamics of the system. Moreover, spins and their associated quasiparticles can also interact with lattice and charge carriers and change the time evolution of the system. ${ }^{16}$

A solid in its non-equilibrium energy state undergoes many relaxation processes to relax back to the thermodynamic equilibrium. Based on their lifetime, these relaxation processes that were explained in the previous paragraphs can be classified into the following sections. In an extremely ultrafast time scale $(<200 \mathrm{fs}$ ), excitation of the solid is in a well-defined phase relation with the 
electromagnetic wave that created the excitation. This stage is called coherent regime. Studying the coherent regime was not possible until recent years because of their extreme ultrafast time scale. The coherent regime includes momentum scattering, carrier-carrier scattering and hole-optical phonon scattering. The first step of destructing the coherence is most probably non-thermal. This means carrier's temperature is so high compare to the lattice that a temperature cannot define their distribution function. This is called the non-thermal regime. Non-thermal processes that happen in the time regime of less than $2 \mathrm{ps}$ include electron-hole scattering, electron-optical phonon scattering and inter-sub-band scattering. To understand the phenomena that bring the non-thermal distribution to a hot thermalized distribution (such as carrier-carrier scattering and exciton-exciton scattering), one should study the non-thermal regime. Next, the carriers or excitons redistribute the energy by a thermalized distribution function that can be characterized by temperature. Collisions within a given electronic system are the main reason for thermalizing a non-thermal distribution. Carriercarrier and exciton-exciton scatterings are the main reason for this thermalized regime, called the hot-carrier regime, that can reach temperatures higher than the lattice temperature. Depending on the interaction type, the hot-carrier regime can last from 1 to $100 \mathrm{ps}$. Thermalization of electrons and holes among each other can takes around 100s ps, while to reach a common temperature it takes about a couple of ps for electrons and holes. By interaction with phonons, these thermalized electron-hole pairs can reach the lattice temperature in 100s ps. This process results in nonequilibrium phonons. Hence, understanding the hot-carrier regime can provide information regarding carrier-phonon, exciton-phonon and phonon-phonon scatterings. After the hot carrier regime is finalized, all carriers, excitons and phonons are in equilibrium with the same temperature. The excess carriers or excitons with respect to the thermodynamic equilibrium recombine either radiatively or non-radiatively. This regime that includes carrier recombination and takes more than $100 \mathrm{~s}$ ps is known as the isothermal regime, which is responsible to return the solid to its thermodynamic equilibrium. It should be noted that these different time regimes can be due to physical processes that are helping different regimes simultaneously. Moreover, the time scale depends drastically on the band structure, type of the excitation and its density, the lattice temperature and excess energy. ${ }^{16}$

Improvement of laser pulses to picosecond pulses in the late 1970s enabled scientists to perform timed resolved Raman spectroscopy to study the dynamics of phonons. As the laser pulses became shorter, direct measurements of reflected, transmitted and absorbed beams enabled the scientists to directly measure the dynamics of the system. By decreasing the laser pulse width to less than 100 femtoseconds, observation of transient change in reflectivity/transmission/absorption due to the change in the refractive index caused by excitation and relaxation of charge carriers, excitons, 
phonons, magnons, etc. became possible. Time resolved spectroscopy has been used to study the dynamics of quasiparticle interactions in different materials such as perovskite oxides.

\subsection{Perovskite Oxides}

Perovskite oxides are a prominent class of functional materials with the formula of $\mathrm{ABO}_{3}$, that can accommodate many of the metallic elements at the A or B sites. In this structure, A is a larger cation compared to B cation. Due to the range of stoichiometry available in the perovskite oxide structure, these materials can exhibit various physical properties. For example, $\mathrm{BaTiO}_{3}$ is a ferroelectric, ${ }^{17} \mathrm{La}_{0.7} \mathrm{Sr}_{0.3} \mathrm{MnO}_{3}$ or $\mathrm{La}_{0.7} \mathrm{Ca}_{0.3} \mathrm{MnO}_{3}$ is a ferromagnet, ${ }^{18}$ and $\mathrm{BiFeO}_{3}$ is a multiferroic. ${ }^{19}$ Moreover, due to the structural properties, many of the perovskite oxides with un-paired outer delectrons form strongly correlated systems. ${ }^{20}$ As a result, many of these oxides are transition metal oxides which based on the spin, charge, lattice and orbital orders, have interesting electronic and thermodynamic properties with many functional applications for the device perspective. Determination of how competition between the degrees of freedom determines the physical properties of the perovskite oxides has been a focus of interest for scientists for years. By improving the experimental techniques, hidden phases in perovskite oxides, especially transition metal oxides, have been observed which enabled these materials for applications in many devices. ${ }^{21}$ This dissertation focuses on static and dynamical optical properties of perovskite oxide LSMO thin films.

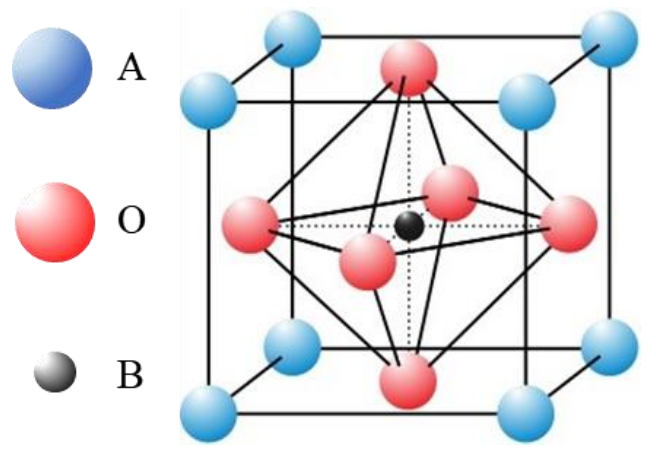

Figure 4. Perovskite oxide's crystal structure.

\subsection{Summary and Goal of Dissertation}

This Ph.D. dissertation started based on the idea to understand the static and ultrafast optical properties of LSMO thin films. In the following chapters, not only are these goals achieved, the extreme cases of ultra-thin films and oxygen pressure deficiencies and their effects on the optical properties have been investigated and addressed. Hence, the central motives of the chapters are formed based on understanding and engineering various optical properties of LSMO thin films. 
Development of a lab-based ultrafast pump-probe setup and optimizing it for LSMO ultra-thin films transient reflectivity was the main experimental challenge in this project. Spectroscopic ellipsometry was also used to understand the static optical properties of the thin films. As a complementary approach, with the help of our collaborators, density functional theory has been used to simulate the optical properties of LSMO thin films.

In the second chapter, the procedure to grow and optimization of high quality LSMO thin films with different growth parameters has been presented. The growth parameters such as thickness and oxygen pressure during the growth have been chosen systematically to address the extreme cases of LSMO thin films.

The third chapter addresses the static optical properties of LSMO thin films in extreme cases. It has been illustrated that in the ultra-thin regime, new electronic transitions result in multiple new peaks in the absorption spectra of LSMO thin films. Moreover, it was observed that by increasing the oxygen content in the thin films, absorption of the thin films increases gradually. To address these new observations in a more systematic approach, DFT calculations suggested what specific electronic transitions can be the result of the new peaks in the optical data.

The fourth chapter which was the main concentration of this dissertation was focused on ultrafast optical properties of LSMO thin films. To the best of our knowledge the ultrafast properties of the thinnest film ever reported was addressed in this chapter. After performing the pump-probe experiment on multiple films with different thicknesses, it was observed that the carriers lose a great portion of their excited energy due to a new recombination mechanism in the ultra-thin regime in a few picoseconds. By comparison to DFT calculations, this recombination was attributed to surface recombination. Moreover, ultrafast spin dynamics of the thin films indicated an ultrafast drop in the spin polarization of the system. This observation suggested light-induced spin polarization lasting for less than one picosecond. Lastly, we performed transit reflectivity measurements on LSMO thin films grown with different oxygen pressure. The effect of oxygen content on the phonon-assisted recombination mechanisms has been illustrated in this chapter.

Finally, in chapter five, we have addressed the transient strain pulse produced at the surface of the thin film as the strong laser pulse hit the surface. Since this strain pulse moves with the sound velocity of the material longitudinally in LSMO thin film, sound velocities in the ultra-thin regime were calculated in this chapter. To achieve this goal, we introduced a new method to study transient strain pulses in ultra-thin films using wavelet analysis. Since dealing with ultra-thin films in this section, we tackle fast vanishing sinusoidal waves. Hence, compared to other studies Fourier 
transform fails to analyze this type of data. Using wavelet analyzes we achieved a sound velocity measurement technique applicable to ultra-thin films with the resolution of monolayers. Based on this technique quantum confinement effect on sound velocities of LSMO ultra-thin films for different film thicknesses have been addressed. 


\section{Chapter 2. Sample Preparation and Characterization}

\subsection{Perovskite Oxide Lao.7Sro.3MnO3 This Films}

LSMO is a perovskite oxide with a general formula of $\mathrm{ABO}_{3}$ consisted of different compositions of $\mathrm{LMO}_{3}$ and $\mathrm{SMO}_{3}$. The crystal structure of LSMO is shown in Figure 5. As indicated in Figure 6 LSMO is a complex oxide with a phase diagram that includes a metal-insulator transition, paramagnetism, antiferromagnetism and ferromagnetism which depend on the Sr dopant level. LSMO exhibits colossal magnetoresistance ${ }^{22-24}$ and prominent ferromagnetism, with a Curie temperature of above room temperature $\left(\mathrm{T}_{\mathrm{c}} \approx 370 \mathrm{~K}\right),{ }^{25}$ and electric properties. Mn's unpaired outer d-electrons increase correlation in LSMO structure which results in LSMO being a strongly correlated material. Hence, these properties can be manipulated by Sr doping level, film thickness, strain effect and oxygen stoichiometry. ${ }^{11,12,26-28}$ It has been indicated that a combination of $70 \%$ LMO and 30\% SMO results in the highest magnetization in the system. Therefore, in this dissertation, we focused on $\mathrm{La}_{0.7} \mathrm{Sr}_{0.3} \mathrm{MnO}_{3}$. The aforementioned composition of LSMO also exhibits half-metallicity in low temperatures, which means metallic properties for one spin channel and insulating properties for the other spin channel. Therefore, LSMO can be useful in spintronic devices. To study the strain effect on LSMO's properties, numerous studies have been done by growing LSMO thin films on different substrates with different lattice parameters such as STO, NGO and LAO. ${ }^{29}$ Many studies have also been performed on LSMO thin films grown in different oxygen pressures to investigate the effect of oxygen vacancies in the system. ${ }^{4}$ The tunability of physical properties enables one to change the band structure, the dopant level and occupancy, resulting in a material that has the potential for light-harvesting, magneto-optic and optoelectronic devices. ${ }^{18,30}$ LSMO is already being proposed in Schottky devices, magnetic tunnel junctions and solid oxide fuel cells. ${ }^{31-33}$

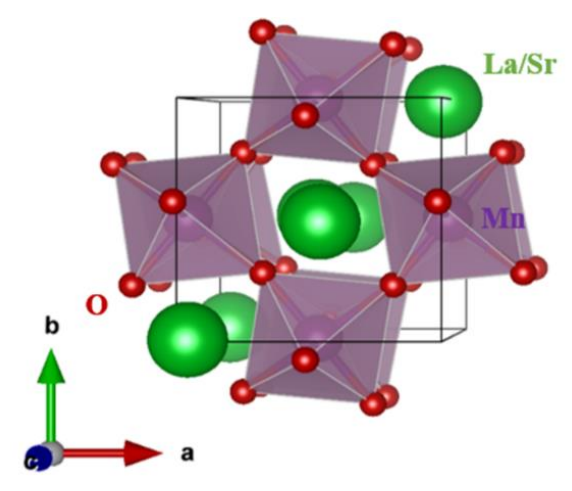

Figure 5. Crystal structure of perovskite oxide LSMO. 
LSMO is a majority hole system $\left(\mathrm{La}_{0.7} \mathrm{Sr}_{0.3} \mathrm{MnO}_{3}=\mathrm{La}_{0.7}^{3+} \mathrm{Sr}_{0.3}^{2+} \mathrm{Mn}_{0.7}^{3+} \mathrm{Mn}_{0.3}^{4+} \mathrm{O}_{3}^{2-}\right)$, resulting in lower curvature of the valance bands. Moreover, Jahn-Teller distortion, which is a common effect in octahedral complexes, plays an important role in the band structure of LSMO thin films. It has been known that by reducing the thickness, metallic and ferromagnetic properties of LSMO decrease drastically. ${ }^{34-37}$ Eventually, LSMO thin films lose their magnetic and metallic properties at a critical thickness called the dead layer. However, the magnetic dead layer is thinner than the metallic dead layer. ${ }^{38,39}$ These dead layers result in hindrance in the functionality of LSMO thin films for device applications. Moreover, it has been shown that the dead layers also exist in thicker films at surface and interfaces. Hence, understanding the surface and interface properties of LSMO thin films is of great importance when it comes to the device's performances. ${ }^{26}$

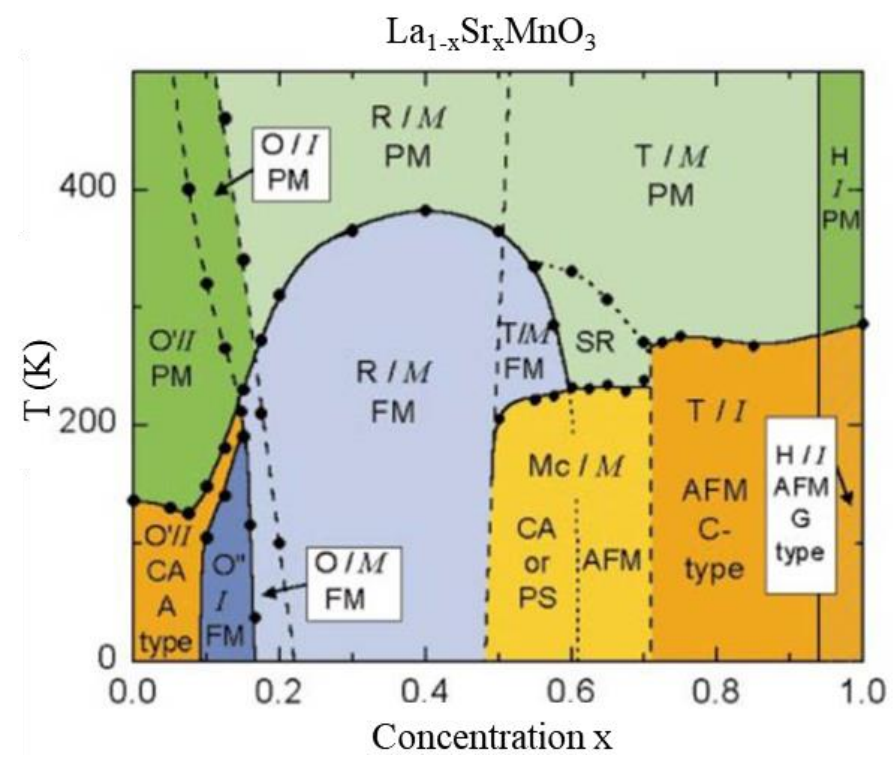

Figure 6. Phase diagram of perovskite oxide LSMO, taken from Hemberger et. al. ${ }^{40}$ Labels are: Orthorhombic (O), Jahn-Teller distorted (O'), orbital-ordered orthorhombic (O”), insulator (I), metallic (M), rhombohedral (R), tetragonal (T), monoclinic (Mc), hexagonal $(\mathrm{H})$, paramagnetic (PM) (green), A-type antiferromagnetic (AFM), ferromagnetic (FM), short-range order (SR), canted (CA), phase-separated (PS). ${ }^{21}$

\subsection{Pulsed Laser Deposition Growth}

To have better device performances, LSMO thin films should be grown with high quality. Since different parameters such as substrate selection, growth temperature and oxygen pressure play an important role in the quality of thin films, achieving this quality is not easy. ${ }^{39}$ Moreover, since a smooth surface morphology is important for most devices, a layer-by-layer growth method is required. Different thin film growth methods have been used to grow LSMO thin films such as molecular beam epitaxy, ${ }^{41}$ chemical solution deposition, ${ }^{42}$ sputtering deposition, ${ }^{43}$ and pulsed laser 
deposition (PLD) ${ }^{44}$ Among these techniques, PLD, which is the method used in this project, is the most used one.

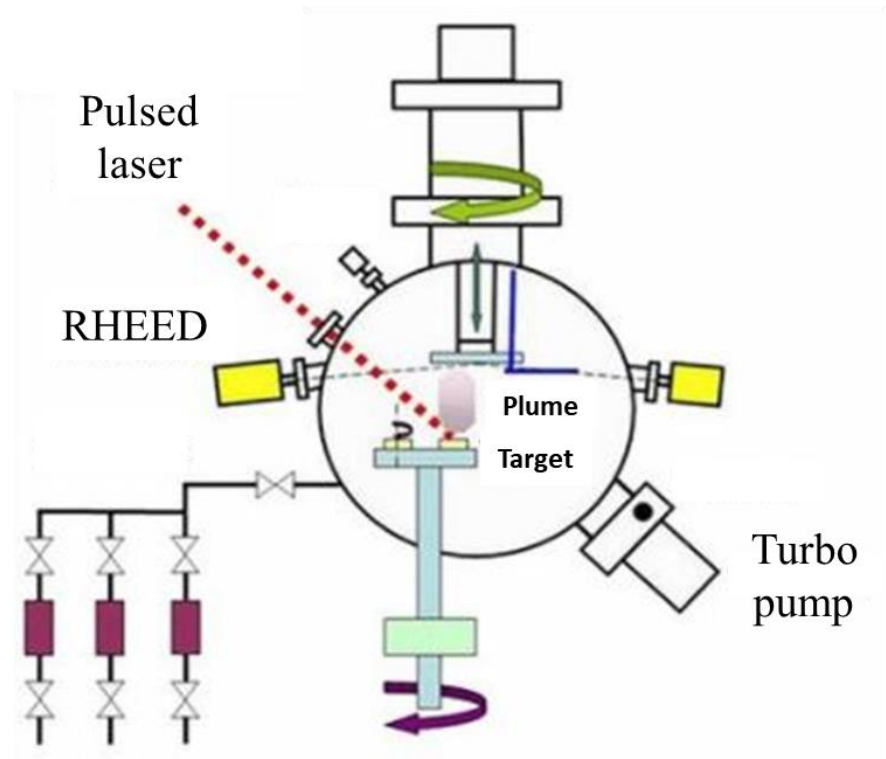

Figure 7. schematic of a pulsed laser deposition system taken from http://www.germantech.com.cn/. ${ }^{45}$

In a PLD system, usually, a focused KrF excimer laser creates a plume of the rotating target material, which is being deposited on the heated substrate. Moreover, a background of desired gases such as Argon, Ozon or Oxygen can be presented at different pressures.

Here perovskite oxide LSMO thin films were fabricated with different thicknesses by PLD on (100) $\mathrm{SrTiO}_{3}$ single crystal substrates. The target material is ablated using a $\mathrm{KrF}$ laser at a wavelength of $248 \mathrm{~nm}$ with an energy fluence of $300 \mathrm{~mJ} /$ pulse. Before growing the sample, the substrates were annealed to form terraces to encourage layer-by-layer growth. Samples were grown under the same optimized conditions of $750{ }^{\circ} \mathrm{C}$ and with various oxygen pressure of 100 to 250 mTorr. After growth, the samples were postannealed in oxygen at 250 mTorr to minimize the presence of oxygen vacancies. Layer-by-layer growth was insured using in situ reflection high energy electron diffraction (RHEED).

\subsection{X-Ray Diffraction}

The structural quality of the thin films was checked with X-ray diffraction (XRD). As a short summary of XRD, this technique can be understood with Bragg's law. If the orientation of the crystal's layer is defined by the Miller indices $(h, k, l)$ and the spacing between each layer in the crystal is $d$, the scattered X-rays, with the incident angle of $\theta$, from each layer interfere 
constructively with each other only if $2 d \sin \theta=n \lambda$. This condition results in different peaks in the scattered X-rays with respect to $\theta$. Each of these peaks will have a specific position, intensity and broadening that can be informative about the arrangement and type of the atoms in the crystal. The position of the peaks is determined by the distance between the parallel layers of atoms. The amplitudes of the peaks are determined on where and what atoms are one the atomic plane. The intensity of the peaks is related to the structure factor $\left(F_{h k l}=\sum_{i=1}^{m} N_{i} f_{i} e^{\left[2 \pi i\left(h x_{i}+k y_{i}+l z_{i}\right)\right]}\right)$ with $I_{h k l} \propto\left|F_{h k l}\right|^{2}$. Here $f_{i}$ is the scattering factor which is equal to the number of electrons around the atoms at $0^{\circ} \theta$ and $N_{i}$ is the fraction of every position that is occupied by atom $\mathrm{j}$. The broadening of the peaks can have information about the sample microstructure, including defects and inhomogeneous composition. ${ }^{46}$

Here we used a Rigaku rotating $\mathrm{Cu}$ anode XRD system to study the crystallinity and quality of the thin films. Figure 8 indicates a $2 \theta$ scan of LSMO thin films. The scans show single orientation LSMO layers with no secondary phases. However, since the lattice parameters of LSMO and STO matches closely, LSMO peaks appear as shoulders on the STO peaks. Moreover, tensile strain causes the peaks to appear to the right side of the substrate peak.

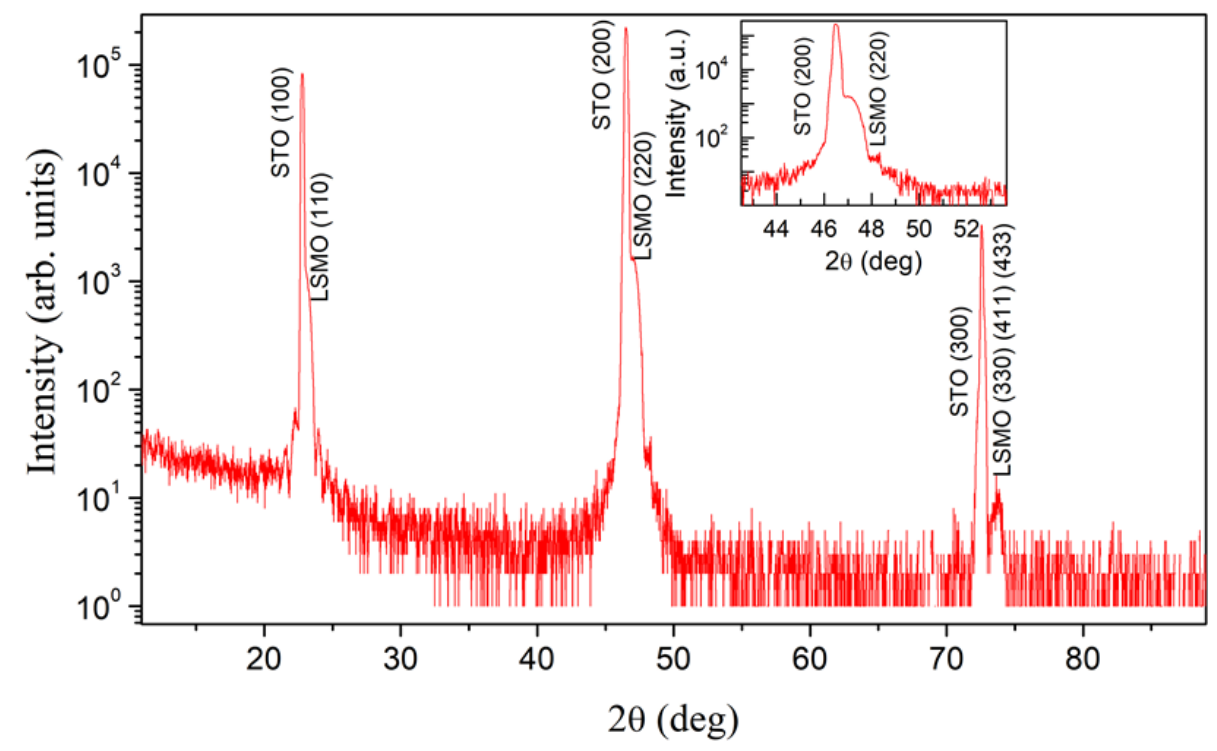

Figure 8. XRD signal for LSMO thin film which shows LSMO peaks as a shoulder next to the stronger STO peak. Inset is the zoomed view of (200) peak.

\subsection{X-Ray Reflectivity}

By performing the XRD experiment at grazing angles, no diffraction is typically observed. Since the thickness is finite in a thin film, reflected X-rays from the top and the bottom surfaces will interfere with each other and result in fringes in the reflectivity signal. As the X-ray incident 
angle increases, the intensity of the reflected beam decreases and eventually the X-ray completely penetrates in the material with reflation going to zero. Different physical properties of the material can be measured using XRR. The oscillation period of the fringes determines the film thickness. The roughness of the surface and interface can be determined by using an envelope to the reflectivity signal as it goes to zero by increasing the incident angle. Here, rougher films will have higher uncertainty and higher reflectivity signal damping coefficient. Moreover, electron density contrast determines the amplitude of the fringes.

GenX $^{47}$ which uses Parrat formalism ${ }^{48}$ and a differential evolution algorithm has been used as a fitting software to analyze the XRR signal. Based on the fitting information the films' thicknesses were confirmed to be 4.4,11.2, 14.1, 20.3, and $43.1 \mathrm{~nm}$. As an example, the XRR result is presented for one thin film in Figure $9 .{ }^{49}$

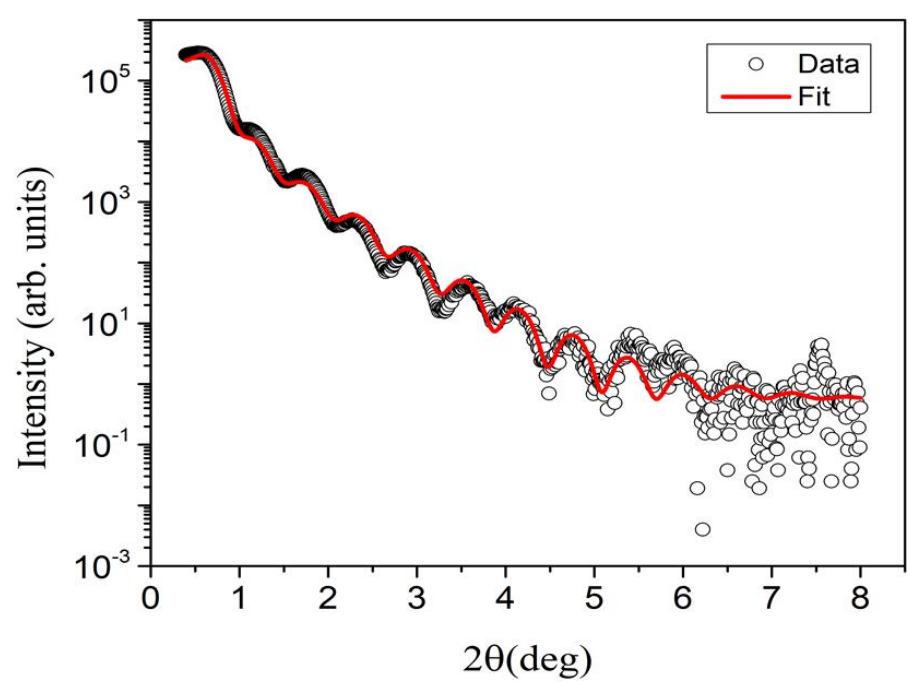

Figure 9. XRR scan and the theoretical fit for 14.1 nm LSMO thin film.

\subsection{Atomic Force Microscopy}

Atomic force microscopy (AFM) is a scanning probe microscopy that can measure the local properties such as height, phase, and magnetism depending on the mode running. The AFM probe is a pyramid with a sharp tip. Hence, the lateral resolution of AFM is low, while its vertical resolution can be as low as $0.1 \mathrm{~nm}$. By measuring the force between the probe and the sample, AFM can provide local information. By reflecting a laser beam off the cantilever and placing the reflected beam on a position-sensitive photodetector, AFM can measure the force and its direction between the sample and the probe. Here we used Asylum MFP-3D AFM in AC mode to gather morphological information regarding the phase and vertical height difference on the sample surface. 


\subsubsection{Substrate preparation}

Due to close lattice match to LSMO, STO single crystalline (100) is a common choice as a substrate, which can result in high quality LSMO thin film growth. Although manufacturers try to provide high quality substrates, prior to growth these substrates should be treated to ensure high quality thin film growth. A common method to decrease the chance of defective growth is forming step terraces on the substrate prior to growth. This will result in better control over the growth process as well as reproducibility, sharper interfaces, and smoother surfaces. Moreover, $\mathrm{BO}_{2}$ and AO terminations on perovskite oxides $\left(\mathrm{ABO}_{3}\right)$, can result in surface roughness and affect the physical properties of the materials. ${ }^{50}$ Without treatment, STO substrates exhibit a mixture of $\mathrm{SrO}$ and $\mathrm{TiO}_{2}$ termination with a flat surface. Hence, fixing the termination of the substrate prior to the growth should also be considered. $\mathrm{TiO}_{2}$ termination of $\mathrm{STO}$ is usually achieved by buffered $\mathrm{HF}$ solution followed by one annealing step. ${ }^{51}$ However, a safer method is to use DI water followed by two annealing steps. ${ }^{52}$ Since $\mathrm{SrO}$ is a basic oxide that is soluble in water, one can achieve pure $\mathrm{TiO}_{2}$ terminated STO substrates using DI water. In order to remove the organic contamination from the surface, the substrates were sonicated in acetone followed by isopropanol each for 5 minutes. The substrates were then soaked in DI water for 5 minutes. This process was followed by annealing the substrate at $1100{ }^{\circ} \mathrm{C}$ for 2 hours. It should be noted that in some cases this process should be repeated to achieve a single terminated step terraced morphology. After the substrate treatment process is done, AFM images can prove a smooth surface with single $\mathrm{TiO}_{2}$ termination and a unit cell step height of $0.4 \mathrm{~nm} .{ }^{49}$
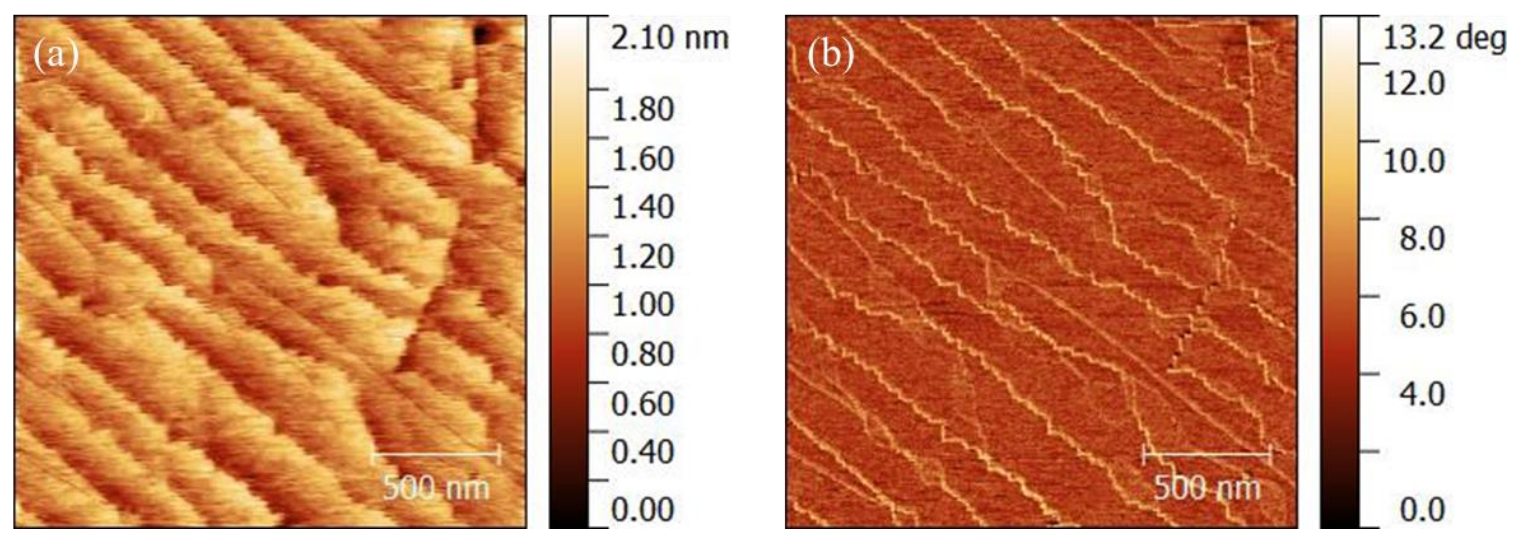

Figure 10. (a) Height and (b) phase AFM images for the STO substrate.

\subsubsection{Thin Film's Surface Morphology}

After the growth, the surface morphology of LSMO thin films taken with AFM exhibits the same step terraces as the one on the STO surface, however, with mix $\mathrm{TiO}_{2}$ termination. ${ }^{53}$ Although 
these terraces are not as sharp and the surface is not as single terminated as STO substrates, having the terraces for an LSMO thin film with a thickness of $38.1 \mathrm{~nm}$ (Figure 11) indicates high growth quality. ${ }^{54}$
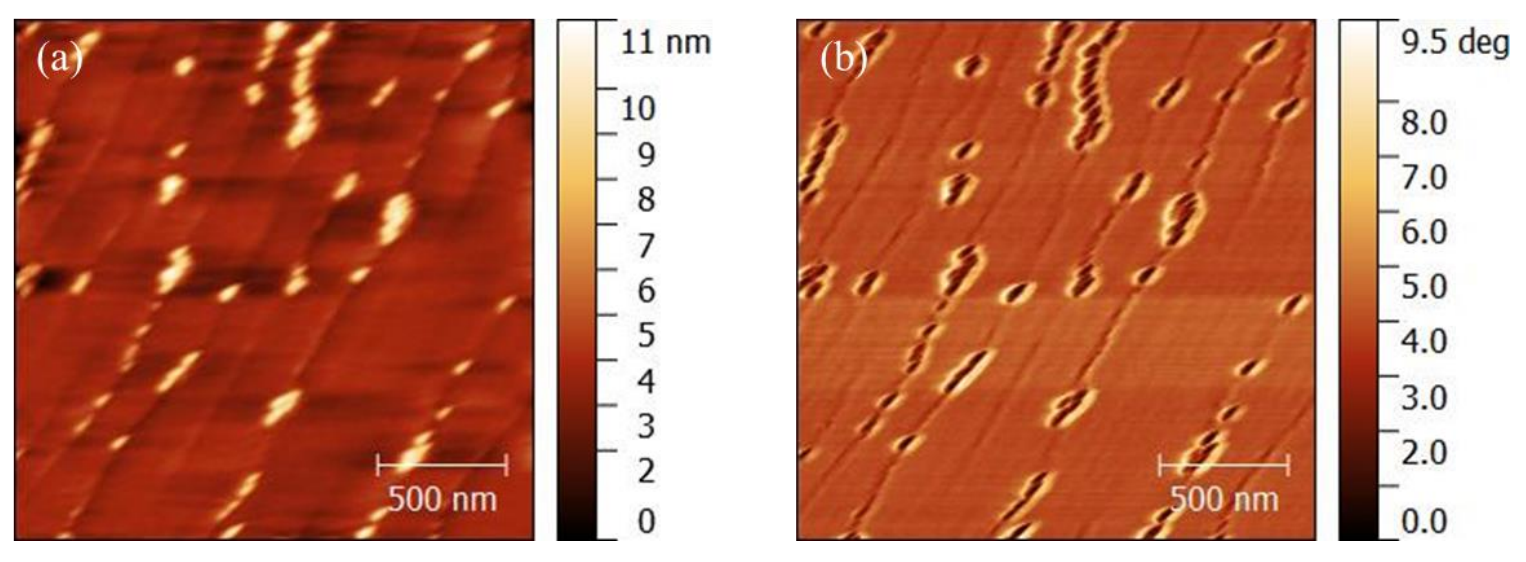

Figure 11. (a) Height and (b) phase AFM images for $38.1 \mathrm{~nm}$ LSMO thin films.

\subsection{Chapter Summary}

Complex oxide thin films' functional properties depend drastically on their growth parameters such as strain, oxygen vacancy and film thickness. Thin-film quality is a major factor that influence the functional properties of oxide thin films such as LSMO. To create high quality single crystalline LSMO thin films, the PLD technique with in-situ RHEED has been used to characterize layer-bylayer growth. After the growth, characterization techniques such as XRD, XRR and AFM are also necessary to assure the quality of the thin films. 


\section{Chapter 3. Static Optical Properties}

\subsection{Introduction}

In semiconductors electrons from filled valance bands can be excited to empty conduction bands by photons with enough energy. Photons can also interact with other quasiparticles in the systems, such as lattice vibrations and electrons localized on defects. When a ray of photons shines on the surface (interface) of a medium, part of the incident beam is reflected, and the remaining is transmitted in the medium. The transmitted portion can be absorbed or scattered by the medium, or pass through the medium. Generally, the reflected and absorbed portions are the strongest processes, since they require the lowest order of interaction between the photon beam and the elementary excitations in the medium. The absorbed portion can be dissipated as heat or reemitted at different energies (photoluminescence). The scattered portion can be due to both static and dynamic inhomogeneities inside the medium. For example, dynamic acoustic waves in the medium can scatter the light and cause Brillouin scattering. Moreover, other elementary excitations such as optical phonons and plasmons can result in Raman scattering. Hence, optical spectra of the semiconductors can provide important information regarding the optical properties of solids. ${ }^{14}$

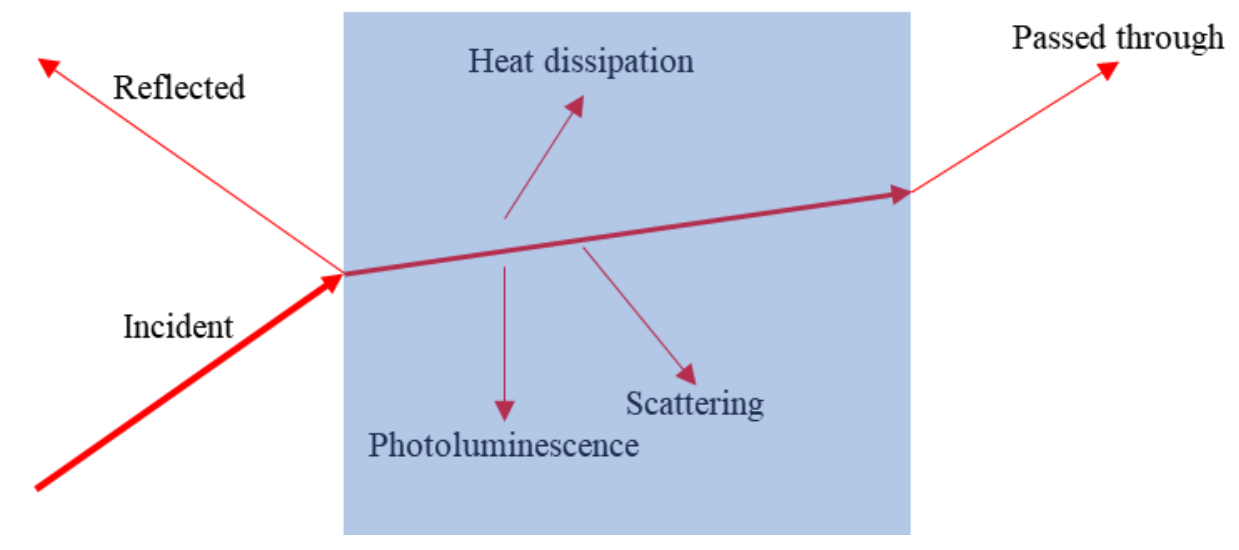

Figure 12. Possible linear optical properties at the surface and bulk of the medium, taken from $\mathrm{Yu}$ et. al..$^{14}$

Understanding optical transitions in complex oxides such an LSMO using their optical spectra is of great importance. LSMO structural, magnetic and optical properties are drastically dependent on oxygen vacancies in the system as well as the concentration of $\mathrm{La}, \mathrm{Sr}$ and $\mathrm{Mn}$. Moreover, in the ultra-thin regime, surface properties of the thin film play an important role in defining those physical properties. Optical spectra of LSMO perovskite oxides can provide information regarding optical transitions between $\mathrm{Mn}^{3+}, \mathrm{Mn}^{4+}$ and $\mathrm{O}^{2-}$ ions. Although there are some studies regarding optical properties of LSMO thin films, ${ }^{1,2,4,4,55}$ a comprehensive study of oxygen vacancies and 
thickness effects on the optical transitions is still missing.

In this chapter, an introduction to spectroscopic ellipsometry as one of the most favorable optical spectra measurement methods in reflectivity configuration has been presented. After explaining the specifics of the measurements, thickness and oxygen vacancy dependence static optical properties have been addressed. It has been indicated that by changing the thickness and oxygen vacancy concentration of the system, some optical transitions' energies have shifted and some of these transitions happen in higher magnitude.

\subsection{Spectroscopic Ellipsometry}

Depending on whether the photon energy is above or below the bandgap, several experimental methods have been suggested to study the optical properties of solids with respect to the photon energy. The imaginary part of the refractive index (the extinction coefficient), which results in absorption, is zero if the photon energy is below the electronic bandgap and well above the phonon energies. However, if the photon energy is above bandgap, the extinction coefficient increases rapidly. Hence, unless the thickness is smaller than the penetration depth of the photon, the system will be opaque for photon energies higher than the bandgap. A common method to measure the complex dielectric function is using a reflected beam. In the case of a high absorption coefficient, the penetration depth of the beam in the solid is less than $1 \mu \mathrm{m}$. Hence, the measurement of the dielectric function using a reflected beam can be very sensitive to the quality of the thin film's surface. Therefore, growing high-quality films in an ultra-high vacuum with a less defective surface plays an important role in this type of measurement. However, the straightforwardness of calculating the dielectric constant from the reflected beam makes techniques based on the reflected beam very popular. ${ }^{14}$

Calculation of the dielectric constant using the reflected beam is based on the Fresnel reflection equations for $\mathrm{S}$ (perpendicular) and $\mathrm{P}$ (parallel) polarized beams. Considering the incident beam from the vacuum (air), on the medium with a complex refractive index of $\tilde{n}$, these equations are as follow:

$$
r_{s}=\frac{E_{r s}}{E_{i}}=\left|\frac{\cos \varphi_{i}-\tilde{n} \cos \varphi_{t}}{\cos \varphi_{i}+\tilde{n} \cos \varphi_{t}}\right|^{2}, r_{p}=\frac{E_{r p}}{E_{i}}=\left|\frac{\cos \varphi_{t}-\tilde{n} \cos \varphi_{i}}{\cos \varphi_{t}+\tilde{n} \cos \varphi_{i}}\right|^{2}, \quad \text { Equation } 1
$$

The oblique incident techniques can measure $r_{s}$ and $r_{p}$. Hence, by knowing $\varphi_{i}\left(=\varphi_{t}\right)$, the complex refractive index, as well as the dielectric constant, can be calculated. Spectroscopic ellipsometry is one of the popular techniques which record the reflected beams in $\mathrm{S}$ and $\mathrm{P}$ 
polarization directions and calculate the optical properties of the materials. ${ }^{56}$

The dielectric tensor of a uniaxial material with only two off-diagonal elements can be written as:

$$
\varepsilon=\left[\begin{array}{ccc}
\varepsilon_{x x} & -\varepsilon_{x y} & 0 \\
\varepsilon_{x y} & \varepsilon_{y y} & 0 \\
0 & 0 & \varepsilon_{z z}
\end{array}\right]
$$

Ampere's and Faraday's laws in Maxwell's equations will result in:

$$
\left(\tilde{n}^{2}-\varepsilon\right) \frac{\omega^{2}}{c^{2}} \vec{E}=0
$$

Hence for a uniaxial material with two off-diagonal elements in the dielectric tensors we have:

$$
\left[\begin{array}{ccc}
\tilde{n}^{2}-\varepsilon_{x x} & -\varepsilon_{x y} & 0 \\
\varepsilon_{x y} & \tilde{n}^{2}-\varepsilon_{y y} & 0 \\
0 & 0 & \tilde{n}^{2}-\varepsilon_{z z}
\end{array}\right]=0, \quad \text { Equation } 4
$$

Which results in two normal modes for $\mathrm{S}$ and $\mathrm{P}$ polarized beam with eigenvalues of:

$$
\tilde{n}_{s, p}^{2}=\varepsilon_{x x} \pm i \varepsilon_{x y}
$$

Based on the Fresnel reflection coefficients and considering complex values for the refractive index, in the general case, the $\mathrm{S}$ and $\mathrm{P}$ polarized waves reflected from the interfaces are complex numbers with different amplitudes and phases. Hence, a combination of these two different components with different amplitudes and phases results in an elliptically polarized wave. This theory forms the basics of spectroscopic ellipsometry which works by detecting the reflected beam.

Ellipsometry is a highly sensitive method to investigate surfaces and interfaces in different materials such as solids, liquids and gases. Since ellipsometry measurements do not require any special surface preparation and can even be done in-situ while the sample is growing, optical properties and thickness measurements using ellipsometry have great advantages compared to alternative methods. ${ }^{57}$

In spectroscopic ellipsometry, the amplitude and phase of the reflected beam for $\mathrm{S}$ and $\mathrm{P}$ polarizations are recorded by performing lock-in measurements at the modulation frequency. This modulation frequency is introduced by a rotating polarizer or a photo-elastic modulator that 
modulates the polarization of the incident beam between $\mathrm{S}$ and $\mathrm{P}$ states.

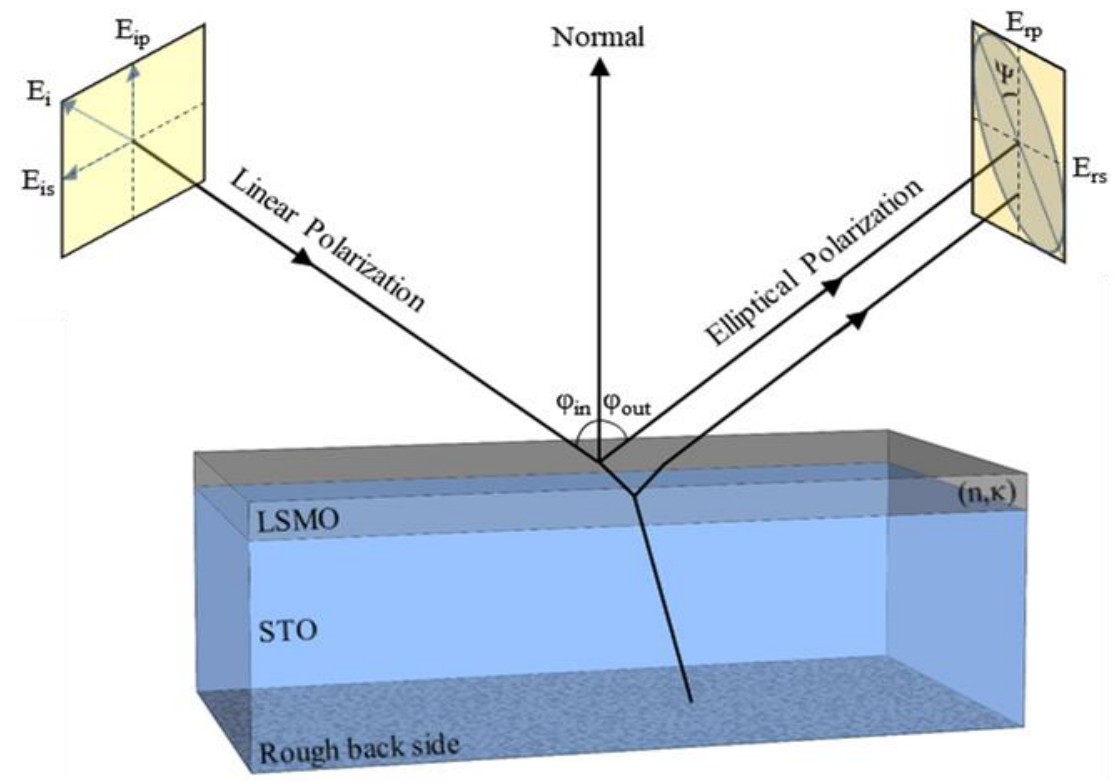

Figure 13. Schematics of spectroscopic ellipsometry.

The polarization state of the ellipse in ellipsometry is described as $\tan \Psi$ and $\cos \Delta$. Here, $\tan \Psi$ is the ratio between the amplitudes of reflected $\mathrm{S}$ and $\mathrm{P}$ polarized waves. Moreover, $\Delta$ is the phase difference between $\mathrm{S}$ and $\mathrm{P}$ components. While $\Psi$ and $\Delta$ are mainly being used to represent an ellipse in spectroscopic ellipsometry, different representations can be used for different purposes. For example, in the magneto-optical Kerr effect (MOKE), ellipticity $(\varepsilon=\tan \gamma)$ and rotation angle $(\theta)$ are used to represent an ellipse. A visualization of all different form of ellipse representation is indicated in Figure 14. ${ }^{58}$

$$
\frac{E_{r p}}{E_{r s}}=\frac{A_{p} e^{i \delta_{p}}}{A_{s} e^{i \delta_{s}}}=\frac{A_{p}}{A_{s}} e^{i\left(\delta_{p}-\delta_{s}\right)}=e^{i \Delta} \tan \Psi,
$$

Equation 6

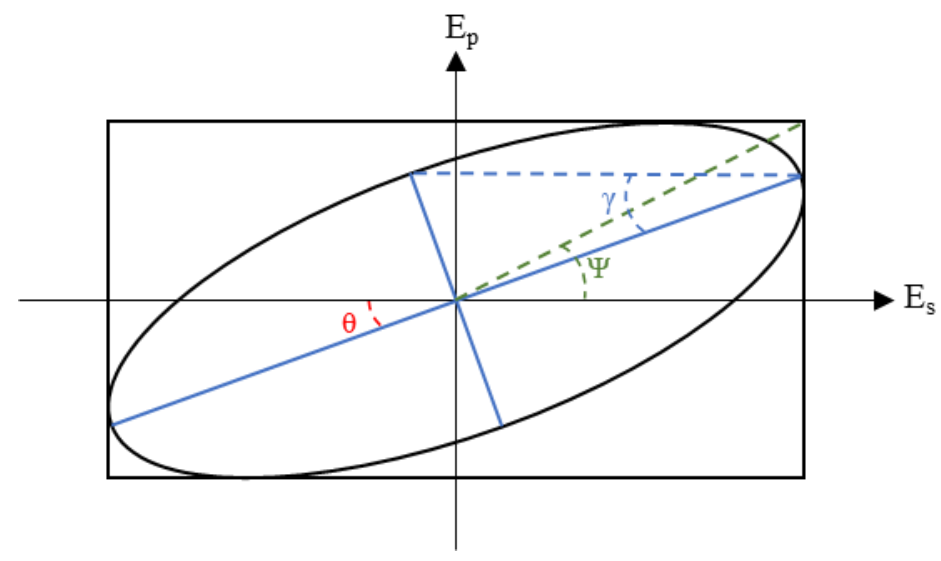

Figure 14. The ellipticity of the reflected beam in spectroscopic ellipsometry. 
Here we used J.A Woolam M-2000U ${ }^{59}$ variable angle spectroscopic ellipsometer (VASE), which performs in the photon energy range of $1.25-5 \mathrm{eV}$, to measure the optical reflection spectra of the thin films. The measurements were carried out in four different incident angles of $60,65,70$ and $75^{\circ}$ in order to improve the modeling process, while the polarization of the incident beam was linear at $45^{\circ}$. After obtaining $\Psi$ and $\Delta$ with respect to the photon energy, this data has been modeled using the CompleteEASE program. ${ }^{60}$ To model the data and extract the refractive index and extinction coefficient, the model contains a pre-defined STO layer as the substrate. The thin film contribution in the data has been modeled in a point by point fashion, using a B-Spline function on top of the substrate. ${ }^{61}$ To avoid overfitting, the thickness and the roughness of the thin films have been dealt with as inputs from XRR measurements. However, since the roughness of the thin films is negligible, the thin films can be modeled with smooth surfaces to simplify the simulations. More details regarding the simulations can be found in the reference. ${ }^{60}$ Care has been taken into account to make sure the substrate backside reflection is not affecting the signal by having a rough substrate backside.

\subsection{Results}

Optical and electronic properties of LSMO have been widely studied with respect to growth conditions such as substrate type, substrate temperature, thickness and oxygen vacancy in the system. ${ }^{1,2,4,42,55,62}$ Having an octahedral environment, the d-orbitals of Mn in LSMO will split into $t_{2 g}$ triplet and $e_{g}$ doublet, due to the crystal field. In LSMO, $t_{2 g}$ bands are lower and narrower, while $\mathrm{e}_{\mathrm{g}}$ are higher and broader. Furthermore, each of these bands are split into spin up and spin down bands. ${ }^{4}$ Hybridization of O-2p and Mn- $\mathrm{e}_{\mathrm{g}}$ bands has also been reported in LSMO near the Fermi level which determines the electronic structure of the system. ${ }^{63}$ Distortion of the octahedron, which is known as Jahn-Teller distortion, also removes the degeneracy of e levels and creates $e_{g}^{1}$ and $e_{g}^{2}{ }^{64}$ LSMO's optical properties are determined by Mn- $e_{g}^{1}$ and $e_{g}^{2}$ and O-2p levels. However, in case of spin-orbit coupling and lattice distortion, the parity and spin forbidden transitions of $t_{2 g}$ to $e_{g}$ and $e_{g}$ to $e_{g}$ can be possible as well. ${ }^{4}$ Being a half-metallic complex oxide, optical properties of LSMO can drastically change in extreme growth conditions. For example, it has been understood that in the ultra-thin regime, LSMO bandgap in the half insulating channel increases. ${ }^{65}$ By increasing the surface to the bulk ratio in the ultra-thin regime, surface states due to broken symmetry on the surface of the thin film, as well as Ti states at the interface, will result in different optical transitions. Hence more features in the optical spectra are expected as the thin film's thickness decreases. Moreover, two extreme conditions of oxygen deficient and oxygen stoichiometric indicate drastically different electronic and optical properties. Each oxygen vacancy in LSMO will result in 
the conversion of two $\mathrm{Mn}^{4+}$ ions to $\mathrm{Mn}^{3+}\left(\mathrm{La}_{0.7}^{3+} S r_{0.3}^{3+} \mathrm{Mn}_{(0.7+2 \delta)}^{3+} M n_{(0.3-2 \delta)}^{4+} O_{(3-\delta)}^{2-}\right)$. This conversion will increase Jahn-Teller distortion. ${ }^{66}$ Hence, more interband optical transitions between $e_{g}$ bands (caused by Jahn-Teller effect) and consequently, more features in the optical spectra are expected.
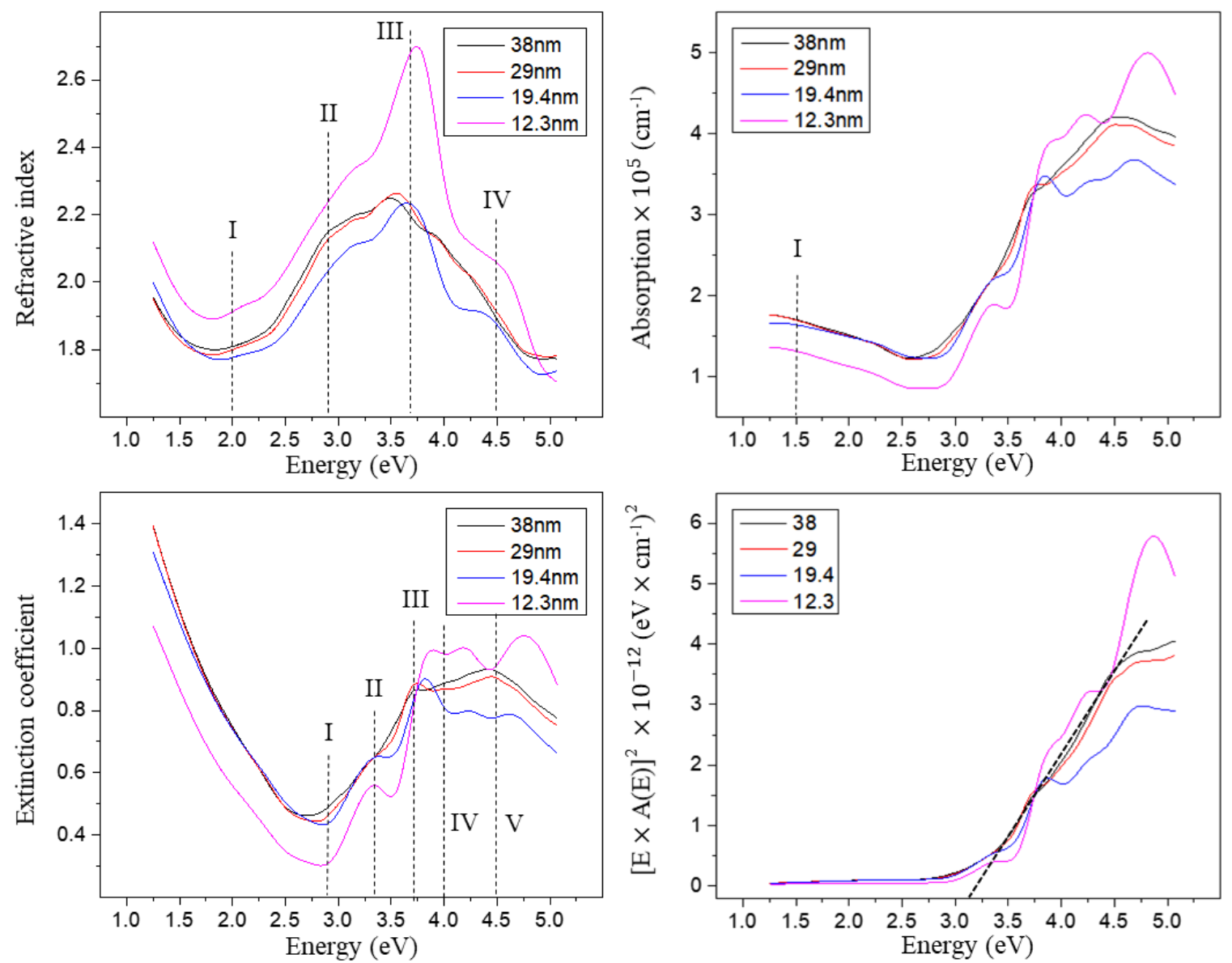

Figure 15. (a) Refractive index, (b) extinction coefficient, (c) absorption, (d) direct Tauc and (e) indirect Tauc plots for LSMO thin films grown with thicknesses of 12.3, 19.4, 29 and $38 \mathrm{~nm}$.

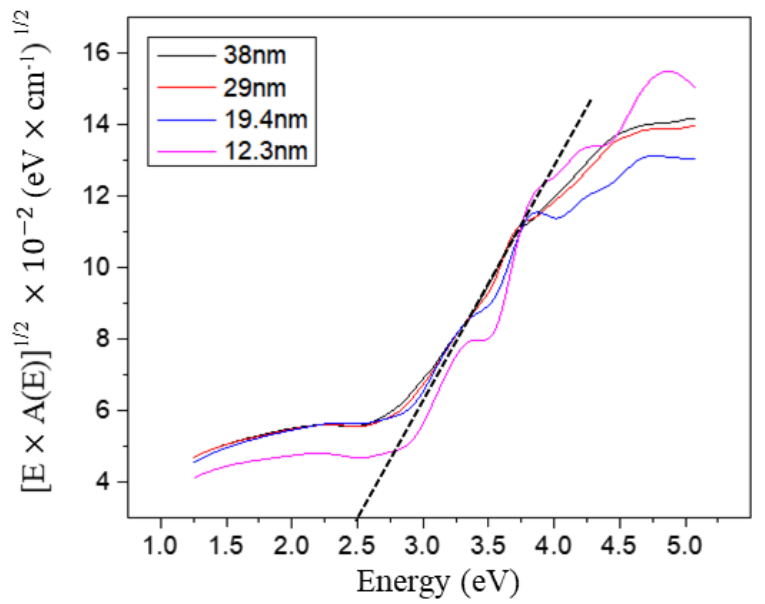

After extracting optical properties of LSMO thin films by modeling the reflectivity data, taken by spectroscopic ellipsometry, refractive index and extinction coefficient of LSMO thin films with 
respect to the photon energy have been presented in Figure 15 (a) and (b), respectively, for films with different thicknesses ( $38 \mathrm{~nm}, 29 \mathrm{~nm}, 19.4 \mathrm{~nm}$, and $12.3 \mathrm{~nm}$ ), under oxygen pressure of 150 mTorr. The absorption spectra of LSMO thin films for different thicknesses have been calculated using $\alpha=4 \pi \kappa / \lambda$ and presented in Figure 15 (c). Figure 15 (d) and (e) indicate the Tauc plots for direct and indirect bandgaps of LSMO thin films respectively. In these plots $[E \times A(E)]^{2}$ and $[E \times A(E)]^{1 / 2}$ have been used for direct and indirect bandgaps respectively.

The refractive index plot indicates multiple peaks that get stronger as the film's thickness decreases. More specifically, broad peaks around $2 \mathrm{eV}$ (I), $2.8 \mathrm{eV}$ (II) and sharper peaks around 3.7 (III) $\mathrm{eV}$ are observable. This plot also indicates particular peaks only for thinner films, which are happening around $4.5 \mathrm{eV}$ (IV). The extinction coefficient plot indicates the possible contribution of off-diagonal elements of the dielectric tensor in broad peaks around $2.8 \mathrm{eV}$ (I), $3.7 \mathrm{eV}$ (III) and $4.5 \mathrm{eV}(\mathrm{V})$. Particularly, Figure 15 (b) indicates further features in the spectra, emerging as the film's thickness decreases, as sharp peaks around $3.3 \mathrm{eV}$ (II), as well as multiple weak sharp peaks with energies more than $4 \mathrm{eV}$ (IV), for thinner films. As indicated in Figure 15 (c) the absorption at $1.55 \mathrm{eV}$, which represents our laser beam's energy, is significantly lower than absorption for energies higher than $3.5 \mathrm{eV}$. Moreover, a sharp drop in the absorption for thinner films at $1.55 \mathrm{eV}$ is visible (I). This low absorption causes a problem when investigating LSMO thin films with laser beams with an energy of $1.55 \mathrm{eV}$ (a typical Ti:Sapphire laser). Eventually, Figure 15 (d) and (e) indicate direct and indirect bandgap of $\approx 3.2 \mathrm{eV}$ and $\approx 2.5 \mathrm{eV}$ respectively, for thicker films.

$\mathrm{Mn}_{\uparrow} \quad \mathrm{Mn}_{\downarrow} \quad \mathrm{O} \quad \mathrm{Ti}$ LSMO under the STO's strain LSMO/STO, 4 u.c. LSMO/STO, 2 u.c.

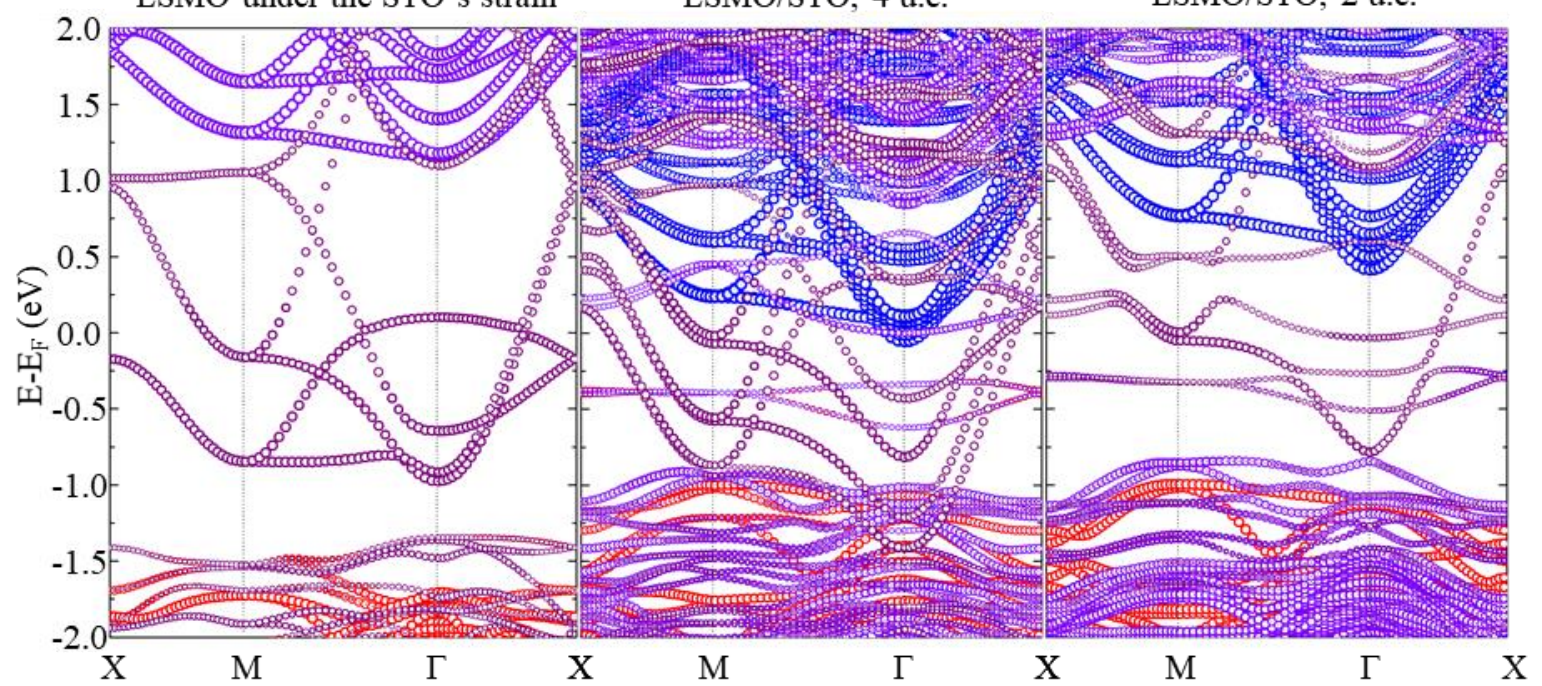

Figure 16. Band structure of LSMO thin films under STO's strain for (a) bulk, (b) 4 unit cells, and (c) 2 unit cells. 
Figure 16 indicates the band structure of LSMO thin films grown on top of the STO substrate, calculated by Dr. Romero's group, which can help to describe these optical features. By decreasing the film thickness, the Ti bands from the substrate affect the optical transitions in the LSMO. Comparing the experimental optical spectra to LSMO band structure, calculated by DFT calculations, some of the peaks presented in the experimental data can be attributed to different optical transitions. Considering the half insulating spin channel in the band structure presented Figure $16(\mathrm{Mn} \downarrow)$, the broad peak around $2.8 \mathrm{eV}$ is due to the transition between O-2p full orbitals and $\mathrm{Mn}-3 \mathrm{~d}$ empty orbitals. ${ }^{67}$ The broad peaks around $2 \mathrm{eV}$ can be attributed to optical transitions between Mn- $\mathrm{t}_{2 \mathrm{~g}}$ and Mn-e ${ }_{\mathrm{g}}{ }^{68}$ Transition between O-2p empty orbitals and Mn- $\mathrm{t}_{2 \mathrm{~g}}$ full orbitals can be the reason for the broad peaks around $4 \mathrm{eV} .{ }^{68}$ Moreover, very weak peaks around $4.5 \mathrm{eV}$ can be due to the transition between O-2p full orbitals and Mn-e empty orbitals. ${ }^{69}$ A slight blue-shift in the optical transitions around $3.7 \mathrm{eV}$ and as well as the strength of this peak, indicates the possible contribution of Ti's band on this peak at the interface. Figure 16 also corroborates this blue shift, since by decreasing the film thickness, the gap at the interface caused by Ti broadens. With the same analogy, strong sharp peaks around $3.3 \mathrm{eV}$ for thinner films, as well as the peaks with the energies more than $4 \mathrm{eV}$ in the extinction coefficient plot can be attributed to optical transitions at the interface between LSMO's bands and Ti's bands.

Figure 17 (a-e) indicate refractive index, extinction coefficient, absorption, direct Tauc and indirect Tauc plots respectively, for LSMO thin films grown in different oxygen pressures. The thickness of all these films are around $30 \mathrm{~nm}$. The positions of the peaks for these oxygen pressure ranges, which are all oxygen stoichiometric, are almost the same. However, the amplitudes of the refractive index, extinction coefficient are changing by oxygen pressure. Figure 17 (c) higher absorptions for LSMO thin films with higher growth oxygen pressures for energies above $2.2 \mathrm{eV}$ (I). On the other hand, the absorption of LSMO thin films decreases for energies less than $2.2 \mathrm{eV}$ as the growth pressures of the films increases (II). As a result, the Tauc plots for direct and indirect transitions, shown in Figure 17 (d) and (e), are exhibiting a slight blue shift in their energies for both direct and indirect transitions by decreasing the growth pressure. 

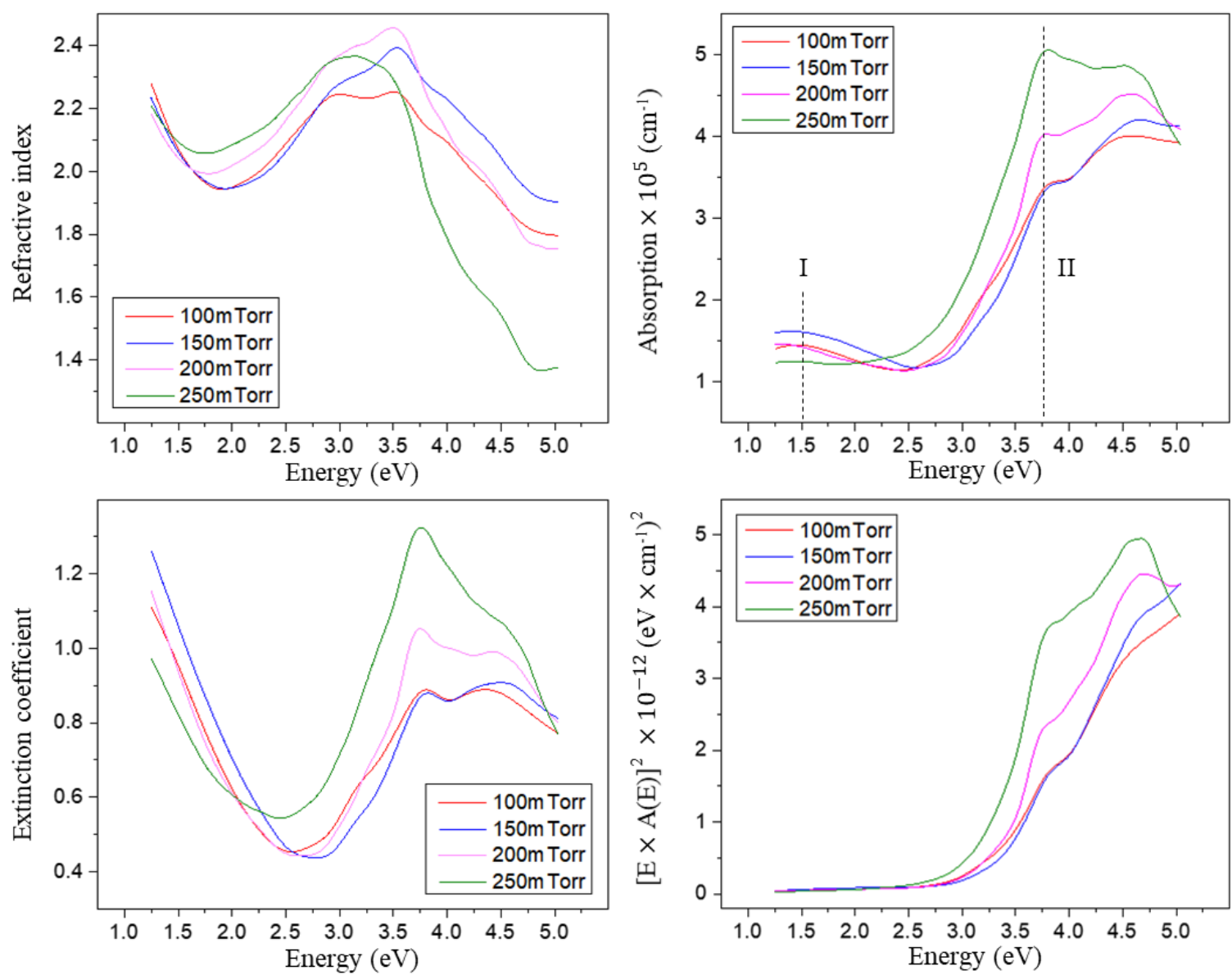

Figure 17. (a) Refractive index, (b) extinction coefficient, (c) absorption, (d) direct Tauc and (e) indirect Tauc plots for LSMO thin films grown under oxygen pressures of 100, 150, 200 and 250 mTorrs.

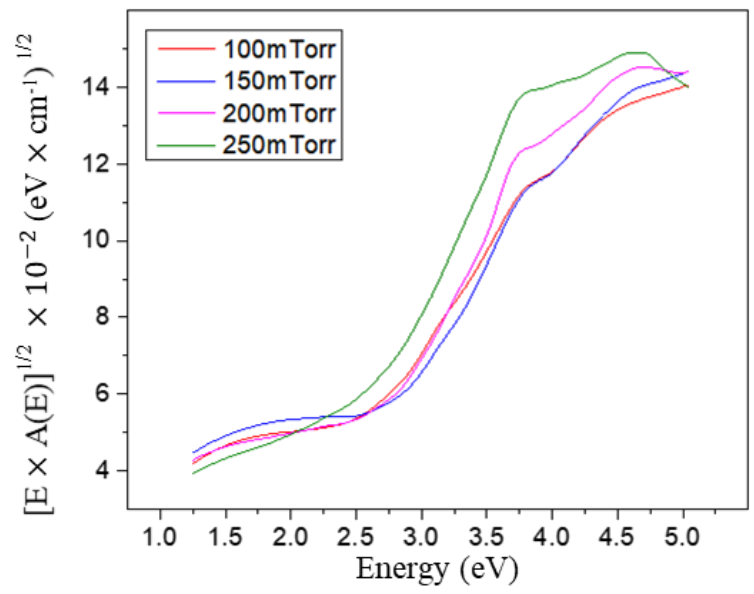

\subsection{Chapter Summary}

In this chapter the static optical properties of LSMO thin films grown under different conditions have been investigated. Optical data taken by spectroscopic ellipsometry indicate multiple peaks for the absorption spectra of LSMO thin film which are dependent on film thickness and growth oxygen pressure. Optical transitions at energies of around 2, 2.8, 4 and $4.5 \mathrm{eV}$ have been observed which are attributed to the transition between $\mathrm{Mn}_{-} \mathrm{t}_{2 \mathrm{~g}} \rightarrow \mathrm{Mn}-\mathrm{e}_{\mathrm{g}}, \mathrm{O}-2 \mathrm{p} \rightarrow \mathrm{Mn}-3 \mathrm{~d}, \mathrm{O}-2 \mathrm{p} \rightarrow \mathrm{Mn}-\mathrm{t}_{2 \mathrm{~g}}$, and 
$\mathrm{O}-2 \mathrm{p} \rightarrow \mathrm{Mn}-\mathrm{e}_{\mathrm{g}}$ respectively. Moreover, transitions at around 3.3 and $3.7 \mathrm{eV}$ which are getting stronger as the film's thickness decreases are attributed to transitions between Ti bands from the substrate and the thin film at the interface. DFT calculations have been performed and indicate the effect of Ti bands on the optical properties of LSMO thin films. Furthermore, in oxygen stoichiometric regime increasing of the growth oxygen pressure results in higher absorptions for energies above $2.2 \mathrm{eV}$ and smaller absorption for energies below $2.2 \mathrm{eV}$. 


\section{Chapter 4. Transient Reflectivity}

\subsection{Introduction}

In the last chapter, the static optical properties of LSMO thin films were addressed. This chapter will be focused on the dynamic optical properties of LSMO thin films. Previous studies on bulk LSMO films have identified electron-phonon relaxation $(\tau<1 \mathrm{ps})$ and phonon-assisted spinlattice relaxation $(\tau \sim 10 \mathrm{~s}$ ps $)$ through the low-temperature-dependent transient-optical response. ${ }^{5,6,70}$ However, there is limited information about this response in thin films near room temperature, which will be relevant for many future devices applications and where differences in ultra-fast dynamics due to surface and interface effects are expected. For example, by decreasing the film thickness, the surface to bulk ratio increases and the surface electrons dominate the features from the bulk electrons. Subsequently, additional energy states (midgap surface states or Tamm levels) emerge between the valence band and the conduction band extrema, providing extra relaxation channels. ${ }^{71} \mathrm{~A}$ thorough study of the surface states is critical for a device application point of view since recombination due to these states drastically affects the efficiency of optoelectronic devices. ${ }^{72,73}$ Moreover, surface effects can oxidize the sample and form defects that can reduce the longevity of the device. ${ }^{74,75}$

In the present chapter, we perform a thickness dependence study of the photocarrier and associated spin dynamics in LSMO thin films, determined by TR measurements with subpicosecond time resolution at room temperature. Variation of the thickness provides insight into changes in the physical properties from LSMO bulk crystals, with a particular focus on effects arising from the additional surface states. For comparison, first-principles calculations and TR experiments on the thicker LSMO films (bulk) reveal large spin-polarity in the optical transitions, occurring through the $\mathrm{O}(2 p) \rightarrow \mathrm{Mn}(3 d)$ transition. While our results on the bulk LSMO films are consistent with previous reports, ${ }^{5,6,70}$ excitation-density dependent TR on thinner LSMO films ( $\mathrm{d}<$ $20 \mathrm{~nm}$ ) reveals a faster relaxation mechanism $(\tau \sim 10 \mathrm{ps})$, which is attributed to the surface recombination. DFT calculations also indicate a significant enhancement in the surface density of states (DOS) with decreasing film thickness yielding an additional surface relaxation mechanism in ultra-thin films. Moreover, the effect of oxygen vacancy, which is one of the most studied topics in oxides, has been investigated in TR signal of LSMO thin films. Our results indicate a drastic effect of oxygen vacancies on phonon-assisted recombination mechanisms.

To understand TR, ultrafast pump-probe spectroscopy has been developed in the lab with different configurations to study the different ultrafast phenomena in the thin film such as free 
carrier dynamics and spin dynamics.

\subsection{Pump-Probe Technique}

\subsubsection{Theory}

As explained in chapter one, the ultrafast dynamics of the system consists of several scattering processes with the timescale of 10s of femtoseconds to 10s of nanoseconds. Detecting such a fast phenomenon requires an experimental technique that has a time resolution smaller than the one of the phenomena. Ultrafast pump-probe setup is a technique to measure transient reflectivity/transmission/absorption indirectly, using ultrashort laser pulses. Depending on the pulse width of the laser, the resolution of this technique can be as low as a few femtoseconds, suitable for measurement of the ultrafast phenomenon in matters. In the pump-probe technique at reflection configuration, a strong pulse (pump) with a duration of around $100 \mathrm{fs}$ excite the system to non-equilibrium states. A second pulse (probe) with a significantly lower intensity will shine on the same spot as the first pulse with a specific time delay and detect the status of the system at that particular time delay after being excited by the first pulse. By sending the probe beam through a delay stage, and introducing different delay times between the pump and the probe beams, the ultrafast dynamics of the system can be scanned directly in the time domain. Depending on the final goal and specific interactions, the pump and probe beams can have different energy levels to detect different phenomenon in the system. For example, the electronic transitions are usually detected with visible or near-infrared laser lights, while the structural changes are detected using ultrashort X-ray pulses. Reflection of the probe beam can provide information regarding the transient reflectivity of the system. Moreover, differential reflectivity can be calculated by subtracting the reflectivity of the ground state form the reflectivity of excited state and normalized by the reflectivity of the ground state $\left(\Delta \mathrm{R} / \mathrm{R}_{0}\right)$.

Transient reflectivity contains information regarding multiple processes in the system, starting with a coherent excitation phase which can decrease or increase the absorption in a few femtoseconds. If the pump pulse energy is equal or slightly higher than the bandgap, it will excite

the carriers to the excited state, leaving the ground state with fewer carriers. In this case, the differential absorption is negative since the absorption of an excited state is less than the ground state. As the band filling effect happens, due to Pauli exclusion, the transition between occupied states in the valance bands and the conduction bands are forbidden, therefore the transition occurs for higher photon energies. Since the real $(n)$ and imaginary $(\kappa)$ parts of the refractive index are related to each other by the Kramers-Kronig relation, the change in the real part of the refractive 
index can be related to the change in the absorption $\left(\alpha=\frac{4 \pi \kappa}{\lambda}\right)$ using: ${ }^{76}$

$$
\Delta n=\frac{2 c \hbar}{e^{2}} P \int_{0}^{\infty} \frac{\Delta \alpha}{E^{\prime 2}-E^{2}} d E^{\prime},
$$

Where $P$ is the principal value of the integral, $e$ is the electron charge, $c$ is the speed of light, and $E=\hbar \omega$ is the photon energy. Therefore, in the case of pump pulse energy equal or slightly higher than the bandgap, the differential reflectivity is negative, as well as differential absorption.

Moreover, when the probe pulse passes through the excited system, stimulated emission will occur for optically allowed transitions. Upon stimulated emission, the same photon as the probe photon will be produced resulting in positive differential reflectivity and consequently negative differential absorption.

If the pump pulse energy is less than the bandgap, some optical transitions happen from the excited states to higher excited states. Absorption of the probe pulse at certain wavelengths will happen due to optically allowed transitions from the excited populated states to higher excited states. Hence, the probe pulse will be absorbed in this situation and the differential absorption will be positive. However, since the intensity of the probe pulse is not high compared to the pump beam, the population of the excited state will remain unaffected. ${ }^{77,78}$

\subsubsection{Experimental}

Figure 18 provides a simplified schematic diagram of the TR geometries used in this dissertation for both circular and linear polarization configurations. TR was performed with a degenerate pump-probe setup using a Spectra-Physics Tsunami Ti:Sapphire laser source at $810 \mathrm{~nm}$ (photon energy of $1.53 \mathrm{eV}$ ) with a repetition rate of $80 \mathrm{MHz}$ and nominal pulse duration of $100 \mathrm{fs}$. The pump pulse was normal to the sample, while the probe had an incident angle of $15^{\circ}$ form the normal. Pump pulses were modulated by a mechanical chopper at a reference frequency of $3 \mathrm{kHz}$ and were focused to a $\frac{1}{e^{2}}$ spot diameter of $50 \mu \mathrm{m}$. To satisfy the overlapping of the two beams, the pump spot diameter was twice the size of the probe spot diameter. A range of pump excitation densities with fluences of $32 \mu \mathrm{J} / \mathrm{cm}^{2}$ to $191 \mu \mathrm{J} / \mathrm{cm}^{2}$ were used in the experiments, meanwhile, the fluence of the probe pulse was kept constant at $3 \mu \mathrm{J} / \mathrm{cm}^{2}$. Detection of the optical signal was recorded in a lock-in amplifier locked to the reference frequency. Transients were measured by varying a mechanical delay stage that has a range of $1.8 \mathrm{~ns}$ with a precision of 66 fs. For linearpolarized configuration, the pump and probe pulses were cross linearly polarized to minimize pump scatter and coherent effects caused by the direct interference of the two pulses at zero delay time. 
A circular pump-probe experiment has been performed to study the spin polarization of the system and spin-dependent dynamics. For circular measurements, additional zero-order achromatic Quartz- $\mathrm{MgF}_{2}$ quarter waveplates with a performing wavelength range of 700 to $1000 \mathrm{~nm}$ quarter waveplates were introduced to perform co- and cross-circular measurements. After reflection, a quarter waveplate was used to change the polarization of the circularly polarized probe to linearly polarized light, which passes through a linear analyzer and reaches the detector. In this setup, a right (left) circular polarized pump beam, RCP (LCP), changes the J-state of the electrons by - $\hbar$ $(+\hbar)$, and the circularly polarized probe beam traces the $m_{j}$ states. ${ }^{7,79}$ Since bulk LSMO is a halfmetallic ferromagnet with more than $90 \%$ spin polarization, almost no minority charge carriers are at the Fermi level at absolute zero temperature. ${ }^{7}$

A non-degenerate setup with various pump and probe wavelengths can be used in the future to focus on different transitions mentioned in the previous chapter. Moreover, in a non-degenerate setup, the scattered pump beam can be filtered using an optical filter, which can simplify the circular pump-probe experiment and make more measurements in the circular configuration possible.

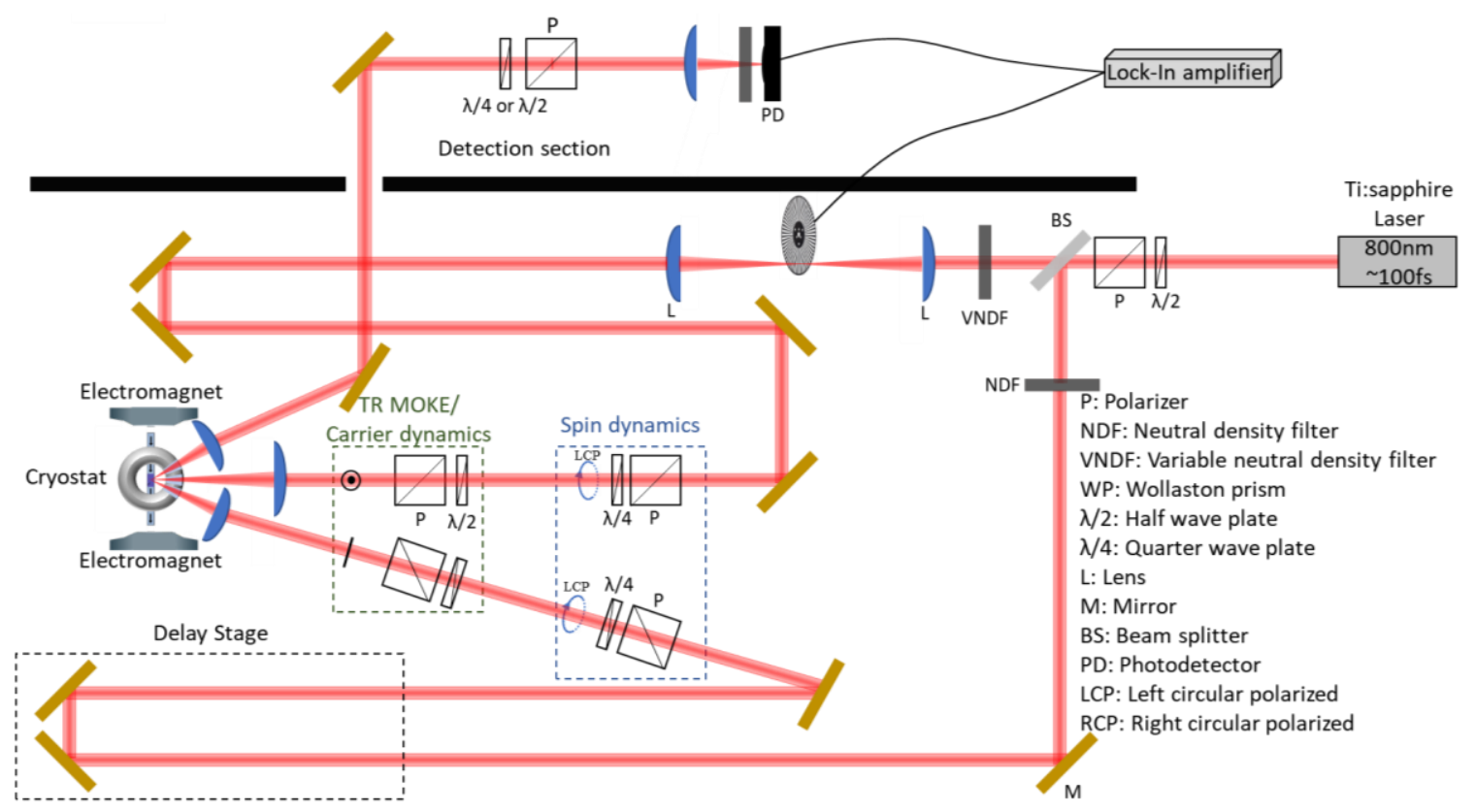

Figure 18. Schematics of ultrafast pump-probe setup.

\subsection{DFT calculations}

To understand the origin and nature of the optical transitions in bulk LSMO, our collaborators in DR. Romero's group performed first-principles DFT+U calculations using VASP code (version 5.3.3) ${ }^{80-82}$ The generalized gradient approximation (GGA) for solids as parametrized by Perdew-Becke-Ernzerhof (PBEsol) was used for the exchange-correlation (XC) functional. ${ }^{83}$ 
The projected augmented wave (PAW) method ${ }^{84,85}$ with a kinetic energy cutoff of $600 \mathrm{eV}$ for the plane-wave basis set was employed. We considered 11 valence electrons of $\mathrm{La}\left(5 s^{2} 5 p^{6} 5 d^{1} 6 s^{2}\right.$, version 06Sep2000), 10 valence electrons of $\operatorname{Sr}\left(4 s^{2} 4 p^{6} 5 s^{2}\right.$, version 07Sep2000), 13 valence electrons of $\mathrm{Mn}\left(3 p^{6} 4 s^{2} 3 d^{5}\right.$, version 02Aug2007), 12 valence electrons of Ti ( $3 s^{2} 3 p^{6} 3 d^{2} 4 s^{2}$, version 26Sep2005), and 6 valence electrons of $\mathrm{O}\left(2 s^{2} 2 p^{4}\right.$, version 08Apr2002) in the PAW pseudopotentials. $\mathrm{La} / \mathrm{Sr}$ concentration ratio of $0.70 / 0.30$ was simulated using the virtual crystal approximation (VCA). ${ }^{86}$ A 9x9x6 Monkhorst-Pack $k$-point mesh ${ }^{87}$ was used for the structural optimization, and a $18 \times 18 \times 12$ Monkhorst-Pack $k$-point mesh was used to calculate the optical transition matrix elements. ${ }^{82}$ To correctly evaluate the strong-correlation effects in LSMO, we employed rotationally invariant $\mathrm{DFT}+\mathrm{U}$ method $^{88}$ with $\mathrm{U}=2.7 \mathrm{eV}$ and $\mathrm{J}=1.0 \mathrm{eV}$, after testing various other combinations of $U$ and $J$ values. Our calculations suggest that the aforementioned $U$ and $\mathbf{J}$ values correctly predict the half-metallicity and the ferromagnetic (FM) ground state of bulk LSMO. Previous studies have also used this combination of $U$ and $\mathbf{J}$ values to correctly reproduce the experimental observations. ${ }^{89,90}$ The PBEsol+U predicted Mn-O bond length to be $1.95 \AA$, which is in excellent agreement with the reported experimental value of $1.95 \AA .55,91$ The optimized lattice parameters for bulk unit cell are $a=5.445 \AA, b=5.461 \AA, c=7.675 \AA$, which are consistent with the reported values in literature. ${ }^{55}$ Having the electronic band structure and optical transition probabilities for different spin channels obtained using DFT calculations, one can relate the results of the TR measurements (electrons relaxation mechanisms) to specific optical transitions for different spin channels and points in the Brillouin zone (BZ).

To study the thickness dependence of surface states in LSMO thin films mounted on the STO substrate, we prepare two slabs consisting of 5 cubic layers of STO and add 4 and 8 layers of LSMO on top of the STO substrate. We add an extra layer of oxygen atoms to complete the Mn-O octahedral at the LSMO surface. A vacuum of thickness $16 \AA$ was added perpendicular to the LSMO surface to avoid any interaction in the periodic geometries. During the structural optimization, the lowest STO layer was frozen to simulate the bulk substrate behavior, whereas all other atoms were allowed to freely relax until the net residual force on each atom was less than $0.001 \mathrm{eV} / \mathrm{A}$. An $8 \mathrm{x} 8 \mathrm{x} 1$ Monkhorst-Pack $k$-mesh was used to sample the reciprocal space of LSMO/STO slabs.

\subsection{Results}

After finalizing the optical setup, the transient reflectivity was performed on LSMO thin films. Figure 19 (a) shows the effect of different excitation fluences, and consequently an increasing number of free carriers, on the free carrier dynamics of $43.1 \mathrm{~nm}$ LSMO on top of the STO substrate. 
The dynamics start with a negative rise in the TR signal as the electrons excite to higher energy states, followed by a decaying signal. This transient can be analyzed phenomenologically by fitting a product of a multi-exponential decay and a Gaussian error function to the data, as follows: ${ }^{92}$

$$
\frac{\Delta R}{R}=\sum_{i=1}^{n} \frac{1}{2} A_{i}\left[1+\operatorname{erf}\left(\frac{t}{\tau_{r}}\right)\right] \times \exp \left(\frac{-t}{\tau_{d_{i}}}\right),
$$

Equation 8

Here, $\mathrm{n}, \tau_{\mathrm{r}}, \mathrm{A}$ and $\tau_{\mathrm{d}}$ are the number of decay components, rise time constant, amplitude and decay time constant of each component respectively. Care was taken to minimize the number of decays while getting an acceptable fit, to avoid overfitting. The MATLAB code used to model the TR data is presented in the appendix.
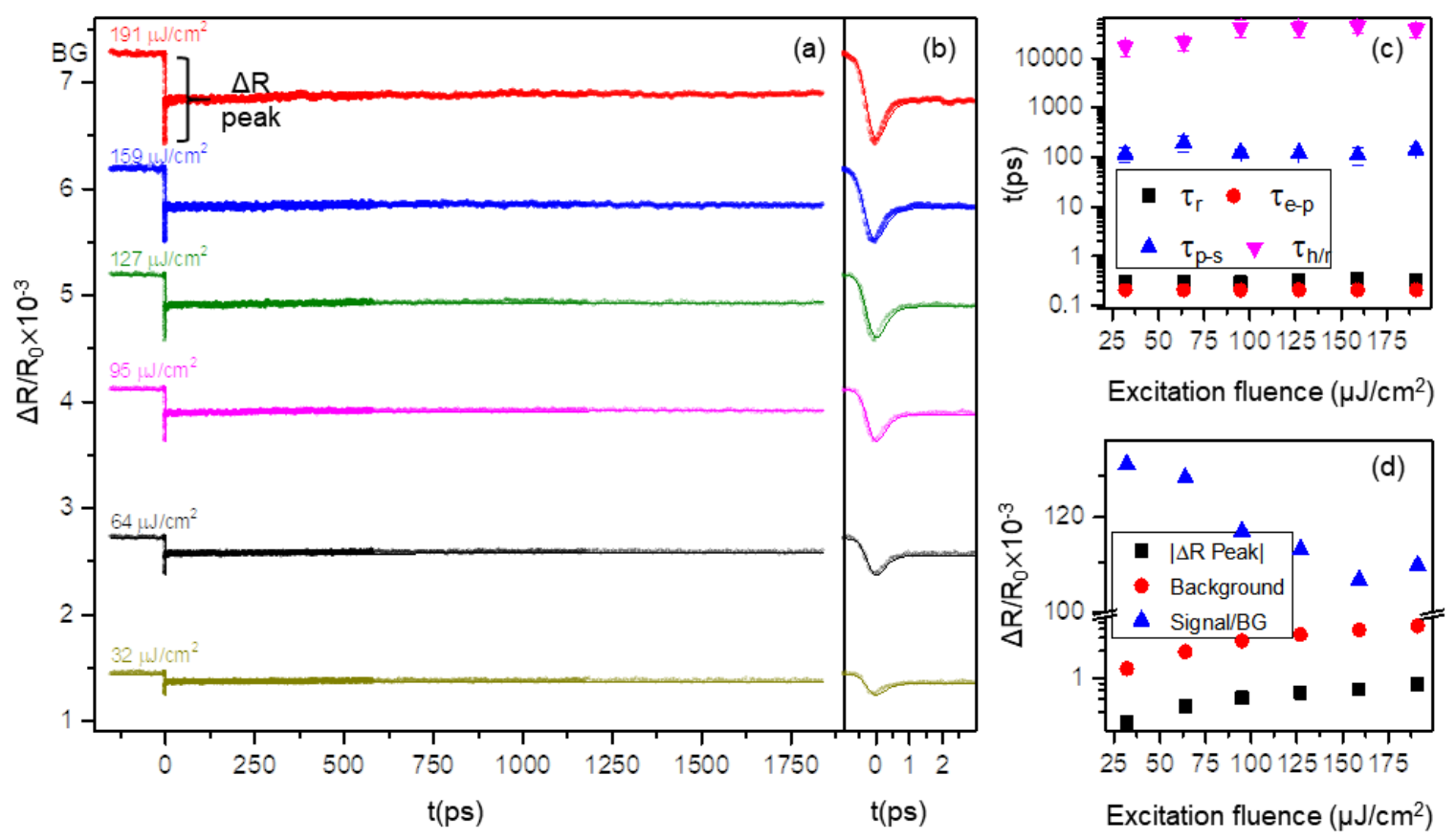

Figure 19. (a) TR measurements for $43.1 \mathrm{~nm}$ LSMO thin film on STO substrate with different excitation fluences. (b) TR measurements zoomed in to the first 3 ps to show the ultra-fast decay due to the electron-phonon interaction. (c) Decay time constants for different decay components as a function of excitation fluence. $\tau_{\mathrm{h} / \mathrm{r}}$ increases with pump fluence, while $\tau_{\mathrm{e}-\mathrm{p}}, \tau_{\mathrm{p}-\mathrm{s}}$, and $\tau_{\mathrm{r}}$ are almost independent of the excitation fluence. (d) The absolute value of $\Delta R / R_{0}$, and background plateau as a function of excitation fluence. While both the background and the peak increase by the fluence, the large difference between the background and peak shows the hindrance in the signal acquisition. The background can be due to coherent effect from scattered pump pulses and/or very slow relaxation mechanism which takes longer than $12.5 \mathrm{~ns}$ when the next pulse excites the electrons again.

For the $43.1 \mathrm{~nm}$ film, the fitting results show one negative rise and three decay components for all the excitation fluence (fit parameters are listed in Table 1). Since the pump photon energy is higher than the energy needed for some optical transitions in half conducting LSMO ${ }^{22,93}$ [Figure 21 
(c)] and lower than the optical bandgap of the substrate (STO), the pump pulse will excite the electron-hole pairs above the bandgap of the film but not of the substrate. The possible excitations shown in Figure 21 (c) result in an absorption decrease due to Pauli blockings as the conduction states fill up, causing the refractive index to decrease, calculated from the Kramers-Kronig integrals. The decrease in the refractive index will result in a negative $\Delta \mathrm{R} / \mathrm{R}_{0}$ signal at $\mathrm{t}=0 .{ }^{18,76}$ This process happens in a sub-picosecond scale ${ }^{19,94,95}$ and corresponds to a fitted rise time $\left(\tau_{\mathrm{r}}\right)$ of $\sim 0.3$ ps, which is independent of the excitation fluence.

Table 1. Fit parameters for multiple decay recombination modeling of LSMO thin films under different excitation powers.

\begin{tabular}{|c|c|c|c|c|c|}
\hline $\begin{array}{c}\text { Excitation } \\
\text { power } \\
(\mathrm{mW})\end{array}$ & $\begin{array}{c}\text { Rise time } \\
(\mathrm{ps})\end{array}$ & $\begin{array}{c}\text { Ultra-fast time } \\
(\mathrm{ps})\end{array}$ & $\begin{array}{c}\text { Fast time } \\
(\mathrm{ps})\end{array}$ & $\begin{array}{c}\text { Medium time } \\
(\mathrm{ps})\end{array}$ & $\begin{array}{c}\text { Slow } \\
(\mathrm{ps})\end{array}$ \\
\hline 300 & $0.32[ \pm 0.01]$ & $0.21[ \pm 0.01]$ & - & $143[ \pm 23.1]$ & $38353[ \pm 12187]$ \\
\hline 250 & $0.32[ \pm 0.01]$ & $0.21[ \pm 0.01]$ & - & $110[ \pm 17]$ & $39451[ \pm 19360]$ \\
\hline 200 & $0.32[ \pm 0.01]$ & $0.21[ \pm 0.01]$ & - & $121[ \pm 25]$ & $41217[ \pm 14701]$ \\
\hline 150 & $0.30[ \pm 0.01]$ & $0.21[ \pm 0.01]$ & - & $126[ \pm 21]$ & $42328[ \pm 15868]$ \\
\hline 100 & $0.30[ \pm 0.01]$ & $0.21[ \pm 0.01]$ & - & $195[ \pm 70]$ & $21166[ \pm 7406]$ \\
\hline 50 & $0.31[ \pm 0.01]$ & $0.21[ \pm 0.01]$ & - & $116[ \pm 37]$ & $17429[ \pm 6242]$ \\
\hline
\end{tabular}

Table 1 provides the fit parameters of the recombination lifetime for the data presented in Figure 19 (a). Based on table 1, Figure 19 (c) provides more insight into the fit results of TR measurements for $43.1 \mathrm{~nm}$ LSMO film. After the negative rise, the electrons (which are now in the conduction band) will couple with the phonons and relax back to the bottom of the conduction band in a sub-picosecond time scale. ${ }^{96}$ The ultra-fast decay time constant $\left(\tau_{\mathrm{e}-\mathrm{p}}\right)$ of $\sim 0.2$ ps is also independent of pump fluence and is consistent with electron-phonon relaxation in LSMO and other similar systems. ${ }^{5,6,19,94}$ The medium time constant $\left(\tau_{\mathrm{p}-\mathrm{s}}\right)$ which is on the order of tens of picoseconds and has previously been proven to be due to phonon-assisted spin-lattice relaxation, ${ }^{5,6,96,97}$ is almost independent of the excitation fluence. The slow time constant $\left(\tau_{\mathrm{h} / \mathrm{r}}\right)$ which is on the order of nanoseconds and is beyond the measurement range of the setup, indicates an increase with increasing the laser fluence. The relaxation phenomena in this timescale could either be due to heat diffusion or slow radiative recombination. ${ }^{96}$ In the first case, there will be a large positive offset at long time delays (compared to the background signal) in the $\Delta \mathrm{R} / \mathrm{R}_{0}$ signal since all the absorbed energy will heat up the lattice. ${ }^{6,19}$ However, for the latter case, only the extra energy after releasing energy (as large as the bandgap) radiatively, will transfer to the lattice as heating and this will cause a negative or a small positive offset in the accessible timescale. ${ }^{19}$ As one can see in the case of our 
study, the offset at long time delays is negative which indicates radiative recombination. On the other hand, the large background shown in Figure 19 (d) which means the electrons cannot release all their energy before the next pulse excites them again ( $12.5 \mathrm{~ns})$, indicates a slow thermal diffusion in the sample. The data presented here is consistent with the data presented in the reference ${ }^{6}$ at low temperatures.

Figure 20 indicates the amplitude of each recombination mechanism with respect to excitation fluence. Increasing the excitation fluence can be interpreted as a higher excited carrier concentration. Hence, the effect of excited carrier concentration on recombinations' amplitudes has been illustrated in Figure 20. The recombination mechanisms become stronger as the excited carrier concentration increases, and eventually, they get saturated.

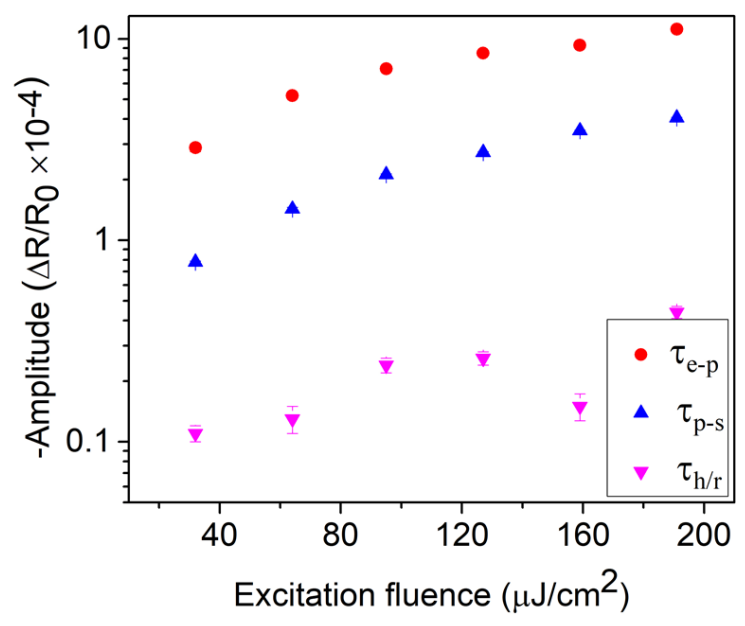

Figure 20. Relaxation mechanism's amplitudes for different excitation fluences.

As indicated in Figure 15 the absorption for ultra-thin LSMO films is drastically low. Parlato et $a l^{6}$ mentioned that at temperatures above $130 \mathrm{~K}$, the signal-to-noise ratio of the LSMO thin film TR signal significantly decreases and to get a less noisy signal one should increase the excitation intensity. However, higher excitation intensity results in sample heating and destruction. As the reference indicated, ${ }^{6}$ a large positive plateau in the signal, which is due to the heated or destructed sample, alters the material's carrier dynamics. This hindrance was overcome by averaging high numbers of scans to reduce the noise statistically. This method enabled us to extract the dynamics even in the presence of considerable noise. The minimum considered number of scans to perform this analysis was 25 for the thickest film and for the highest excitation intensity, while it was 50 for thin samples. The noise to signal ratio presented here is comparable to the one reported in reference ${ }^{6}$ at low temperature. The background magnitude increases linearly with the pump fluence. The peak magnitude in the signal also monotonically increases with the pump fluence. However, this increase 
is not as strong as the increase in the background due to the excitation fluence. This difference in the slopes makes the signal for lower pump fluences less noisy [Figure 19 (d)].

The half-metallicity of the films results in having a much stronger transient signal for one spin channel and a very weak transient signal for the other spin channel, at zero Kelvin. It is expected that by increasing the temperature, the half-metallicity of the system decreases. Performing spin dynamic measurements using a similar pump-probe setup with circularly polarized light proves the significant half-metallicity of the system if electrons are excited only for one spin component. Figure 21 (a) represents spin dynamic measurements for different excitation powers. As expected for half-metallic thin films at room temperature, the excitation is stronger for one of the spin components (in this case pump $_{\mathrm{LCP}}$-probe $\mathrm{RCP}_{\text {) }}$ compare to the other one ( pump $_{\mathrm{LCP}}$-probe $\mathrm{LCP}_{\mathrm{LC}}$ ). The first inset of Figure 21 (a) indicates the time-resolved degree of spin polarization, calculated from $P=\frac{I_{R}-I_{L}}{I_{R}+I_{L}}$, for different excitation powers (I). By increasing the excitation power, the degree of spin polarization increases. ${ }^{98}$ This observation suggests that spin polarization in the system is mainly photoinduced. Analyzing the polarization degree using a multi-component decay indicates two decay components, one of which has a lifetime of a few femtoseconds while the other one has a lifetime of hundreds of picoseconds, indicated in inset (II). The amplitude of each component is presented in inset (III) indicating the dependence of each component's strength on the excitation fluence. Inset (III) indicates that the slower component (C2) is exhibiting a strong dependence on the excitation fluence which indicates strong photoinduced polarization in a slower time regime. Meanwhile, in ultrafast time regime $(\mathrm{C} 1)$, the polarization is not completely photoinduced. By decreasing the excitation fluence, eventually, the slower decay component (C2) vanishes and the spin polarization of the system completely turns off after the ultrafast decay component $(\mathrm{C} 1)$ is completed. Since for the ultrafast component (C1) we observe the same dynamics as the carrier dynamics from the linear polarization configuration, one can relate all the dynamics in LSMO halfmetallic thin films to the spin systems. ${ }^{79,99}$ As can be seen in Figure 27 (b) and Figure 20, the contribution of the electron-phonon interactions in LSMO free carrier dynamics is the strongest contribution. These elastic scatterings play an important role in spin relaxation in higher temperatures. ${ }^{100}$ Hence, the stronger sub-picosecond recombination $(\mathrm{C} 1)$, that the same time regime as the electron-phonon interactions, has been attributed to electron-phonon interactions in Figure 19 and Figure 20, indicating that by increasing the temperature (excitation intensity), the intensity of spin relaxation due to electron-phonon interactions increases. 

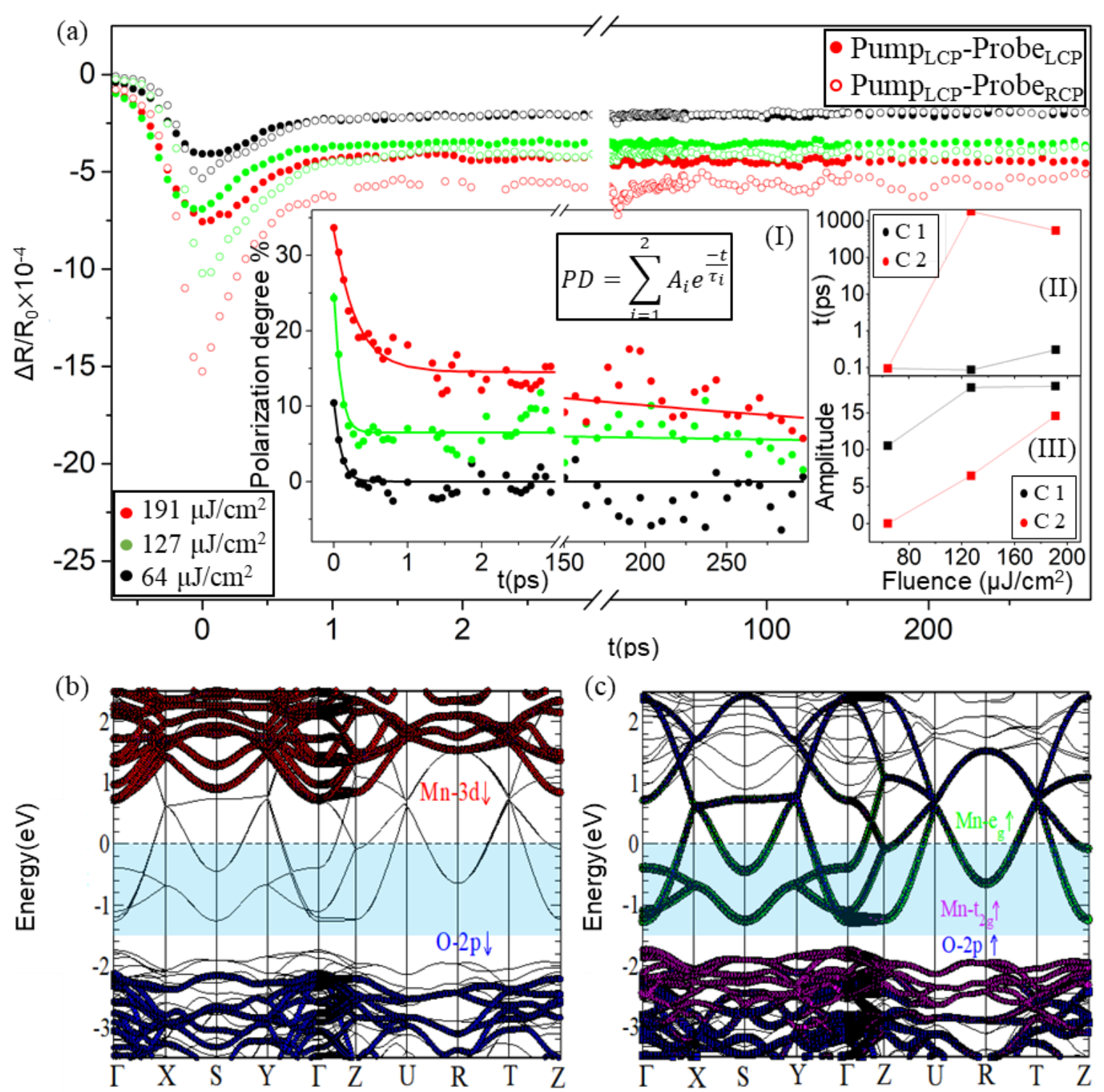

Figure 21. (a) Spin dynamics measurements for different excitation powers at room temperature. Inset (I) indicates the degree of spin polarization for different excitation powers modeled by twocomponent decay model. Insets (II-III) represent the lifetime and amplitude of each decay component $(\mathrm{C} 1$ and $\mathrm{C} 2)$ with respect to excitation fluence respectively. Orbitals projected electronic band structure for (b) minority spin-channel and (c) majority spin-channel. The shaded area depicts the energy window of $1.53 \pm 0.01 \mathrm{eV}$ which is the range of the excitation energy of the used laser pump pulse.

Figure 21 (b-c) represents the orbital projected electronic band structure of LSMO for minority and majority spin-channels, calculated along the high symmetry directions of the BZ shown in Figure 21 (c). The projection of minority spins on the electronic bands reveals the insulating feature for the minority spin-channel with an energy gap of $\sim 2.85 \mathrm{eV}$, whereas metallic features can be observed for the majority spin-channel. The shaded area in Figure 21 (b-c) depicts the energy 
window of $1.53 \pm 0.01 \mathrm{eV}$ below the Fermi-level, marking the region from where the pump pulse $(810 \mathrm{~nm})$ used in our TR measurements could potentially excite the charge carriers to the unoccupied states. For the minority spin-channel, the possible transitions could occur only from the occupied $\mathrm{O}(2 p)$ states to the empty Mn (3d) states. However, these transitions cannot occur in our experiments because the used photon energy is smaller than the optical bandgap of the minority spin channel.

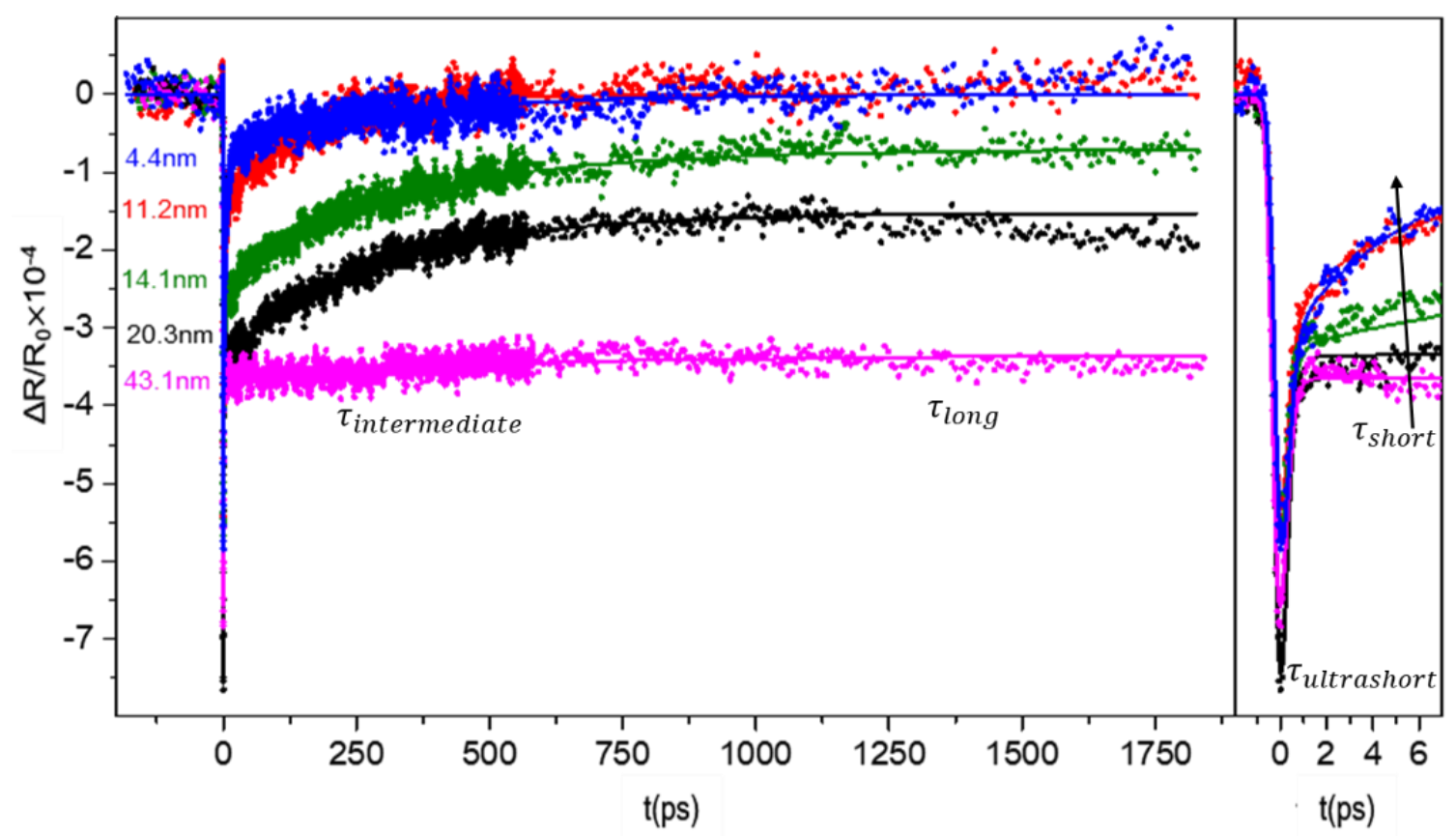

Figure 22. TR measurements for LSMO thin films with different thicknesses. For thinner films, an extra relaxation mechanism was observed. For the $14.1 \mathrm{~nm}$ film, all four dynamics were observed, however thinner films lose all their energy after hundreds of picoseconds and no loss of energy due to heat diffusion or radiative recombination was observed.

Figure 22 shows the TR measurements results after normalizing the background on LSMO samples with different thicknesses. While films with thickness above $\sim 20 \mathrm{~nm}$ have the same type of dynamics, thinner films show an extra relaxation mechanism emerging as the LSMO film becomes thinner (fit parameters are given in Table 2 and Table 3). TR measurements were performed on LSMO thin films of various thicknesses, with the excitation and probe fluence of 191 $\mu \mathrm{J} / \mathrm{cm}^{2}$ and $3 \mu \mathrm{J} / \mathrm{cm}^{2}$ respectively. For $43.1 \mathrm{~nm}$ and $20.3 \mathrm{~nm}$ thin films, the fit was converged with three decay components in ranges of sub-picosecond, tens and thousands of picoseconds. However, for films thinner than $11.2 \mathrm{~nm}$ the range of the three decay components, which gave the best fit results, were in the range of sub-picosecond, a few and hundreds of picoseconds. For $14.1 \mathrm{~nm}$ LSMO thin film three decay components model failed to fit the data. Adding an extra decay 
component solved this problem and resulted in four decay components in the range of subpicosecond $\left(\tau_{\mathrm{e}-\mathrm{p}}\right)$, a few $\left(\tau_{\mathrm{s}}\right)$, tens $\left(\tau_{\mathrm{p}-\mathrm{s}}\right)$ and thousands of picoseconds $\left(\tau_{\mathrm{h} / \mathrm{r}}\right)$. Having a new mechanism emerging as the surface electrons start to dominate the bulk electrons, to study the emergence of the new phenomena, a new version of the fit, with four decay components, were performed to all the data to be thorough regardless of signs of overfitting (i.e. very small amplitudes, the same decay time constant, etc). As a result of overfitting for some of the films, larger error bars have appeared [Figure $22(\mathrm{a}-\mathrm{b})$ ], and the fit with the optimum number of decay components (three) with acceptable error bars are presented in Figure 23.
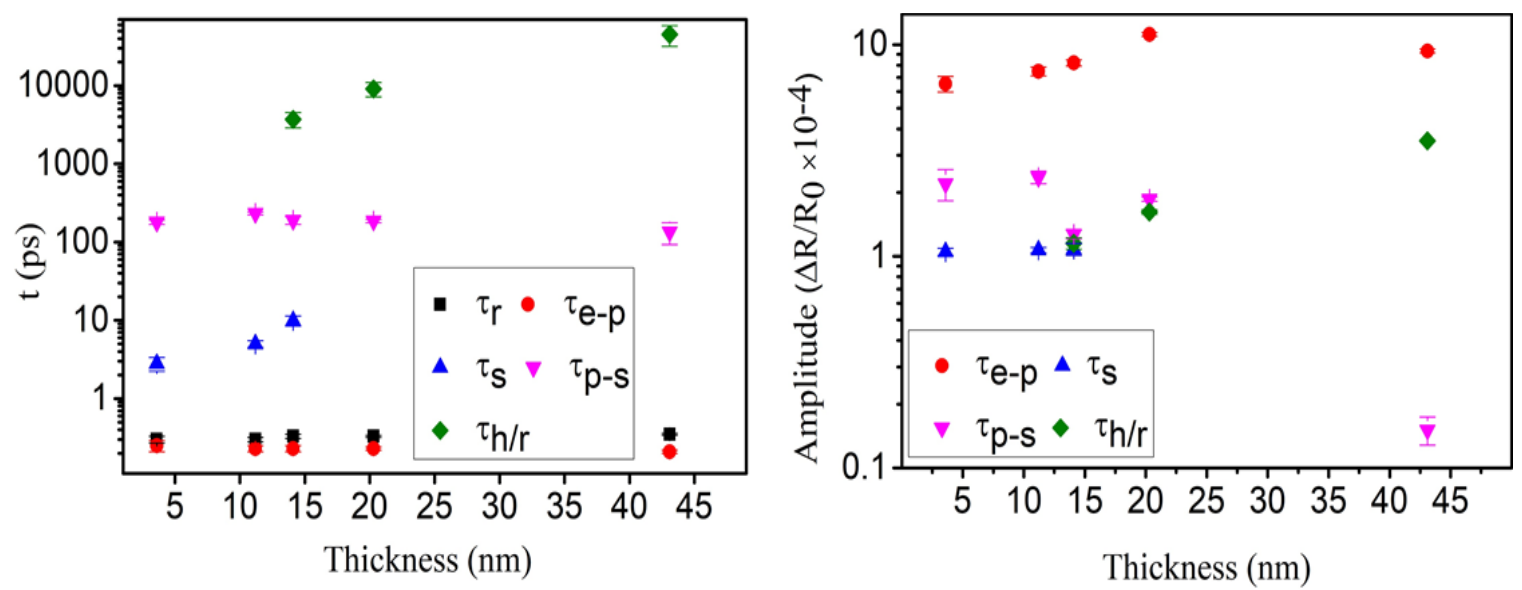

Figure 23. Relaxation mechanism's time constant (a) and amplitudes (b) for LSMO thin films with different thicknesses.

As a result of fitting with four decay components, Figure 24 (a-b) show the decay time constant and amplitude of each decay component. While the rise, $\tau_{\mathrm{e}-\mathrm{p}}$ and $\tau_{\mathrm{p}-\mathrm{s}}$ are almost independent of the film thickness, $\tau_{\mathrm{s}}$ and $\tau_{\mathrm{h} / \mathrm{r}}$ increase with the film thickness. The amplitude plot [Figure 24 (b)] shows the electrons lose most of their energy via interaction with phonons, since the magnitude of the amplitudes for the electron-phonon decay components (red) are almost an order of magnitude higher than the other components. The other three decay components almost have the same contribution to the electrons' energy relaxation. By decreasing the film thickness the quantum confinement becomes a matter of great importance. The general increase in the electron-phonon relaxation amplitude with thickness shows that the bandgap decreases with thickness and the interaction with phonons become less effective in larger bandgaps (thinner films) which is consistent with quantum size effect for strongly correlated LSMO half-metal system. ${ }^{65,101}$ Figure 24 (a) indicates a separate mechanism $\left(\tau_{\mathrm{s}}\right)$ as the thickness of the film decreases, however, this mechanism becomes indistinguishable from the phonon-assisted spin-lattice recombination as the film thickness reaches its bulk value. We attribute this separate mechanism to surface 
recombination since it happens when the surface to bulk ratio increases. Moreover, as discussed in the reference, ${ }^{71}$ since the lattice mismatch between the substrate and the film is small, ${ }^{89}$ unwarranted interface traps are negligible. The decrease of the surface recombination time constant $\left(\tau_{\mathrm{s}}\right)$ by decreasing the thickness indicates the path length for an electron to reach the surface to recombine with the surface states reduces, resulting in a faster recombination time constant in thinner films. Figure 24 (b) shows the strong effect of surface recombination in thinner films (less than $\sim 20 \mathrm{~nm}$ thick) and in these films, electrons lose most of their energy without any radiative or thermal recombination. On the other hand, as the thickness increases, since the bulk carrier density dominates the surface carrier density, the surface recombination appears to be negligible with respect to the other decay components. In the thicker films (above $\sim 20 \mathrm{~nm}$ thick) strong radiative or thermal recombination are responsible for the energy loss in the electrons.

Table 2. Relaxation time constant fit parameters for multiple decay recombination modeling of LSMO thin films TR signal with different thicknesses.

\begin{tabular}{|c|c|c|c|c|c|}
\hline $\begin{array}{c}\text { Thickness } \\
(\mathrm{nm})\end{array}$ & Rise time $(\mathrm{ps})$ & $\begin{array}{c}\text { Ultra-fast } \\
\text { time }(\mathrm{ps})\end{array}$ & Fast time $(\mathrm{ps})$ & $\begin{array}{c}\text { Medium time } \\
(\mathrm{ps})\end{array}$ & Slow (ps) \\
\hline 3.6 & $0.37[ \pm 0.03]$ & $0.25[ \pm 0.05]$ & $1.6[ \pm 0.38]$ & $96[ \pm 24]$ & $727[ \pm 229]$ \\
\hline 11.2 & $0.34[ \pm 0.01]$ & $0.23[ \pm 0.02]$ & $5.0[ \pm 0.89]$ & $70[ \pm 12]$ & $509[ \pm 152]$ \\
\hline 14.1 & $0.33[ \pm 0.02]$ & $0.23[ \pm 0.02]$ & $9.88[ \pm 1.46]$ & $190[ \pm 21]$ & $3710[ \pm 833]$ \\
\hline 20.3 & $0.34[ \pm 0.01]$ & $0.23[ \pm 0.01]$ & $71[ \pm 36]$ & $286[ \pm 111]$ & $9298[ \pm 6507]$ \\
\hline 43.1 & $0.34[ \pm 0.01]$ & $0.21[ \pm 0.01]$ & $134[ \pm 3796]$ & $360[ \pm 4235]$ & $25699[ \pm 39838]$ \\
\hline
\end{tabular}

Table 3. Amplitude constant fit parameters for multiple decay recombination modeling of LSMO thin films TR signal with different thicknesses.

\begin{tabular}{|c|c|c|c|c|}
\hline Thickness (nm) & $\begin{array}{c}\text { |Ultra-fast } \\
\text { amplitude| }\end{array}$ & $\begin{array}{c}\text { |Fast } \\
\text { amplitude| }\end{array}$ & $\begin{array}{c}\text { |Medium } \\
\text { amplitude| }\end{array}$ & $\begin{array}{c}\text { |Slow } \\
\text { amplitude| }\end{array}$ \\
\hline 3.6 & $5.79 \mathrm{E}-4[ \pm 7.5 \mathrm{E}-5]$ & $2.48 \mathrm{E}-4[ \pm 6.6 \mathrm{E}-5]$ & $6.6 \mathrm{E}-5[ \pm 1 \mathrm{E}-5]$ & $4.5 \mathrm{E}-5[ \pm 1.1 \mathrm{E}-5]$ \\
\hline 11.2 & $7.38 \mathrm{E}-4[ \pm 3.3 \mathrm{E}-5]$ & $1.57 \mathrm{E}-4[ \pm 1.5 \mathrm{E}-5]$ & $1.1 \mathrm{E}-4[ \pm 9 \mathrm{E}-6]$ & $3.8 \mathrm{E}-5[ \pm 1 \mathrm{E}-5]$ \\
\hline 14.1 & $8.2 \mathrm{E}-4[ \pm 2.7 \mathrm{E}-5]$ & $1.08 \mathrm{E}-4[ \pm 7 \mathrm{E}-6]$ & $1.27 \mathrm{E}-4[ \pm 6 \mathrm{E}-6]$ & $1.15 \mathrm{E}-4[ \pm 7 \mathrm{E}-6]$ \\
\hline 20.3 & $0.00111[ \pm 2.2 \mathrm{E}-5]$ & $5 \mathrm{E}-5[ \pm 3.3 \mathrm{E}-5]$ & $1.27 \mathrm{E}-4[ \pm 2.1 \mathrm{E}-5]$ & $1.75 \mathrm{E}-4[ \pm 2 \mathrm{E}-5]$ \\
\hline 43.1 & $9.44 \mathrm{E}-4[ \pm 1.8 \mathrm{E}-5]$ & $2 \mathrm{E}-6[ \pm 1.31 \mathrm{E}-4]$ & $1 \mathrm{E}-5[ \pm 9.8 \mathrm{E}-5]$ & $3.52 \mathrm{E}-4[ \pm 3.9 \mathrm{E}-5]$ \\
\hline
\end{tabular}

Table 2 and Table 3 provide the fit parameters of recombination's lifetime and amplitude for the data presented in Figure 22 respectively. As presented in these two tables, due to the very small amplitudes of different components, the error bars are high for this data set. 

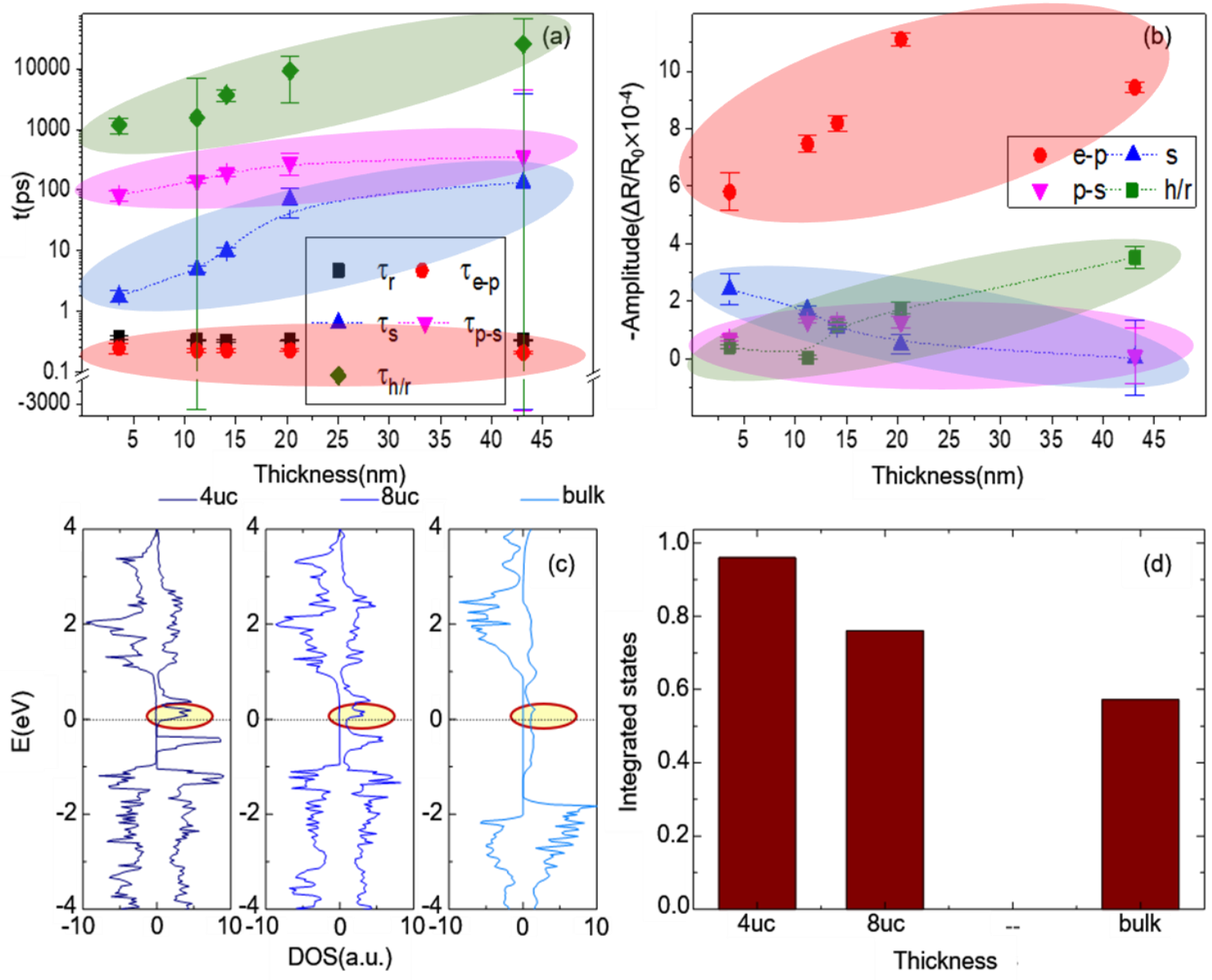

Figure 24. (a) Decay time constants for different films showing the surface recombination time constant (blue dots) increases with the thickness. (b) Negative amplitudes of decay components showing the energy dissipated by the surface recombination decay component are comparable to the lost energy due to $\mathrm{p}$-s and heat diffusion/radiative recombination. The surface recombination becomes stronger as the films become thinner. (c) DOS for 4 and 8 unit cells LSMO thin films compared to bulk LSMO, indicating the density of surface states increase by decreasing the film thickness. (d) integrated DOS in the energy window of $[-0.25,0.25] \mathrm{eV}$.

Figure 24 (c) represents the calculated surface DOS for 4 and 8 unit cells LSMO thin films mounted on a 5 unit cells STO substrate. In the same figure, we also present the DOS for bulk LSMO for comparison. We notice an increase in the DOS near the Fermi level as the thickness decreases, thus indicating a higher density of surface states in thinner films. Figure 24 (d) shows the integrated DOS in the energy window of $[-0.25,0.25] \mathrm{eV}$, further suggesting a systematic increase in the surface DOS in thinner films close to the Fermi energy. Having a higher density of surface states results in higher transition probability through surface states and consequently a higher amplitude for the surface recombination in thinner films which is consistent with the fit results in Figure 24 (b). Moreover, the averaged electric potential $\left(\frac{\partial V_{z}}{\partial z}\right)$ along the vertical direction 
rapidly increases with decreasing thickness of deposited LSMO films, as reported by Trappen et $a l$. in a recent work. ${ }^{89}$ This causes an increase in the electric field along a direction perpendicular to the LSMO surface leading to more energy band bending near the surface ${ }^{102,103}$ and consequently faster surface recombination of charges in thinner films which is consistent with the fitting results presented in Figure 24 (a).

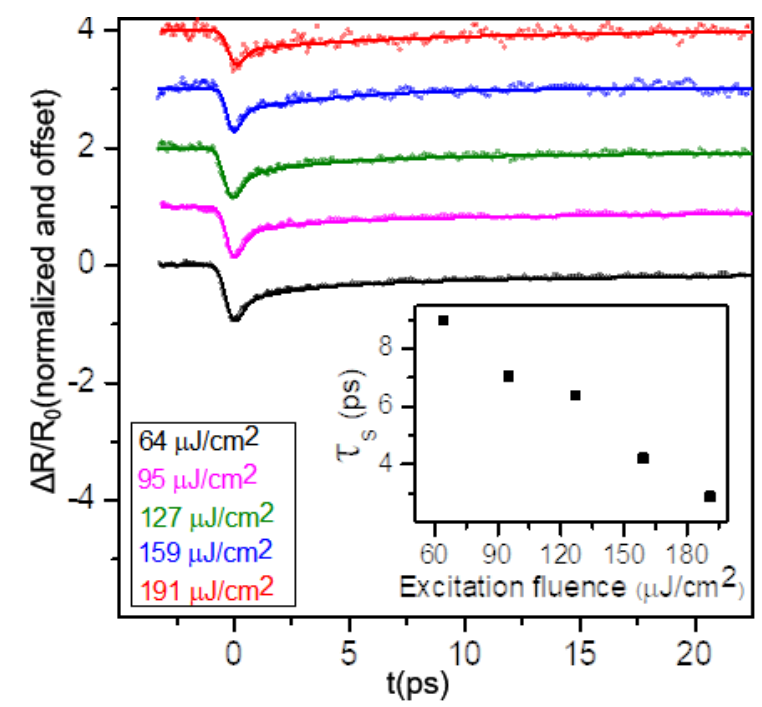

Figure 25. Excitation fluence dependence for $4.4 \mathrm{~nm}$ LSMO thin film shows that the surface recombination decay time constant decreases with laser fluence.

Figure 25 shows TR measurements for different excitation fluences for $4.4 \mathrm{~nm}$ LSMO thin film with the focus on the surface recombination. The inset of Figure 25 indicates the dependence of the decay lifetime of the surface relaxation mechanism with pump fluence for the $4.4 \mathrm{~nm}$ LSMO thin film. There is a decrease in the decay lifetime with increasing excitation fluence. This decrease can be qualitatively understood by utilizing the relations among excitation power, temperature and the kinetic energy of charge carriers. According to Lin, ${ }^{104}$ the maximum pulsed laser temperature varies almost quadratically with the laser power $(\mathrm{P})$, i.e. $\mathrm{T} \propto \mathrm{P}^{2}$. After photoexcitation by a femtosecond laser pulse, because of the large difference between the electronic and lattice (phononic) heat capacities, the excess laser energy creates a non-equilibrium electron distribution and leaves the lattice temperature almost unchanged. In a femtosecond timescale the non-equilibrium electrons redistribute their energies among themselves via electron-electron interaction. This process results in an electronic temperature, much higher than the phononic temperate $\left(T_{e}>T_{P}\right)$, leaving the system with two different temperatures. In a widely separate timescale (picosecond regime), carriers with a much higher temperature of $\left(\mathrm{T}_{\mathrm{e}}\right)$ cool down by emitting phonons. Due to the large temperature difference between the electrons and the lattice (tow temperature model), after the picoseconds electron-phonon interaction, the temperature equilibrium process results in lower final electronic 
temperature and higher final lattice temperature, compared to their initial temperature, respectively. In the case of higher laser power, more excited carriers will result in a higher initial electronic temperature. The higher initial electronic temperature will eventually (after the picosecond regime electron-phonon interaction) increase the equilibrium temperature between the electrons and the lattice. Therefore, by increasing the laser power the equilibrium temperature and final thermal velocity of the carriers will increase. The kinetic energy of carriers of effective mass $m$ and velocity $v$ can be expressed as:

$$
\frac{1}{2} m v^{2} \propto \frac{3}{2} k_{B} T_{e} \propto P^{2}
$$

Equation 9

where, $k_{B}$ is the Boltzmann constant. Therefore, the higher the laser power, the higher the surface recombination velocity, and thus shorter time $(t \sim 1 / v)$ for recombination. ${ }^{71}$ This decrease in the surface recombination time constant is consistent with the surface recombination velocity since by increasing the thin film temperature (due to the laser power increase), surface recombination velocity increases. Consequently, electrons on the surface recombine faster, ${ }^{71}$ suggesting ultra-thin LSMO films as a suitable material for the future ultra-fast optoelectronic and magneto-optic devices.
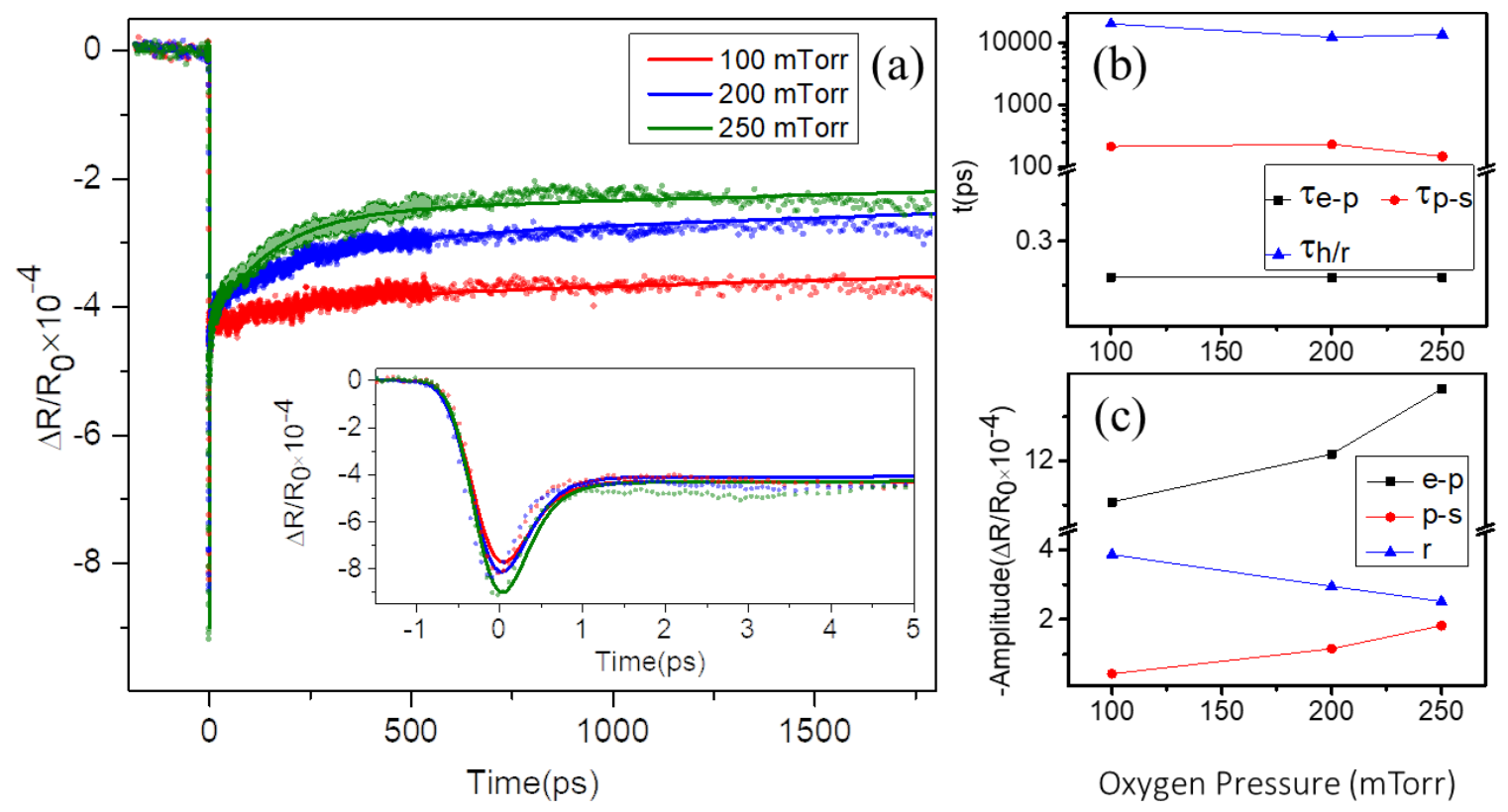

Figure 26. (a) Carrier dynamics for $30 \mathrm{~nm}$ LSMO thin films with different growth oxygen pressure. (b) The time constant and (c) the amplitude related to each recombination mechanism.

As discussed previously, differences in oxygen vacancies in complex oxides can create changes in many physical properties such as structural and magnetic properties. Figure 26 (a) indicates 
ultrafast carrier dynamics for $30 \mathrm{~nm}$ LSMO thin films grown under 100, 200 and 250 mTorr Oxygen pressure in the PLD system. As shown in Figure 26 (a), excited carriers in LSMO thin films with higher oxygen pressure (i.e. fewer oxygen vacancies) recombine to the initial states faster. The inset of Figure 26 (a) indicates stronger excitation for LSMO thin films grown in higher oxygen pressure (i.e. fewer oxygen vacancies). Figure 26 (b) indicates almost no relation between the recombination time and the oxygen pressure in the system, for all three recombination mechanisms. However, Figure 26 (c) indicates the amplitudes of the recombination mechanisms are strongly dependent on the oxygen vacancies in the system. (surface recombination has been neglected here since the thickness of the films are high) In higher growth oxygen pressures, by introducing more electrons in the system, the electron-phonon recombination's amplitude increases. Moreover, Figure 26 (c) indicates that by increasing the growth oxygen pressure, the next phonon-assisted phenomena (i.e. spin-lattice recombination) also become stronger. However, the carriers that have lost most of their energies by the two mechanisms of electron-phonon and spin-lattice recombination, in higher growth oxygen pressures (i.e. fewer oxygen vacancies) will now have less energy to recombine back by the means of thermal recombination. Hence, the thermal recombination amplitude is lower in higher oxygen pressures.

\subsection{Chapter Summary}

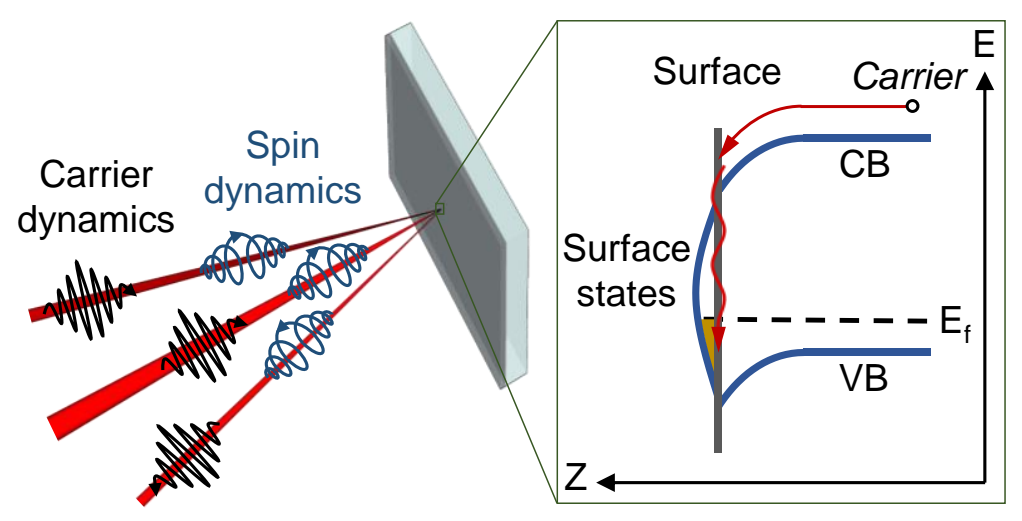

Figure 27. Schematics of surface recombination in ultra-thin films.

Surface recombination at room temperature in complex oxide LSMO ultra-thin films which can significantly affect device applications has been studied in this work. The TR measurement at room temperature for films above $\sim 20 \mathrm{~nm}$ thick, is consistent with results already published in the literature at lower temperatures. These measurements indicate three relaxation mechanisms. (i) electron-phonon recombination ( $\tau<1 \mathrm{ps}$ ), (ii) phonon-assisted spin-lattice recombination $(\tau \sim 100$ ps) and (iii) thermal diffusion/radiative recombination ( $\tau>1 \mathrm{~ns})$. Circular TR measurements 
indicate that due to LSMO's half-metallic behavior, the dominant carrier dynamics come from the conducting spin majority channel, while almost no response is observed from the insulating spin minority channel. DFT calculations enabled us to determine the possible optical transitions, indicating major optical transitions occurring in the majority spin channel. Reducing the film thickness, enabled the study of the surface effect on LSMO thin films as the surface contribution increases with respect to the bulk contribution in thinner films. Consistent with the experimental observation of surface recombination for films thinner than $\sim 20 \mathrm{~nm}$, DFT calculations indicate an increase in the density of the surface states in thinner films which consequently results in stronger surface recombination. Reduction in the path length from the bulk to the surface and increase in the energy band bending near the surface results in faster surface recombination in thinner films. Moreover, the surface decay time constant monotonically decreases with increasing excitation fluence, indicating higher carrier velocity for higher excitation fluences. In addition to film thickness, oxygen enhancement in LSMO thin film have also drastic impact on the carrier's lifetime. Our observation indicates an enhancement in phonon-assisted recombination's amplitude which eventually results in lower carrier's lifetime. 


\section{Chapter 5. Coherent Acoustic Phonons}

\subsection{Introduction}

The interaction of an ultrafast laser pulse with solids excites quasiparticle interactions between the electrons, spins, phonons, magnons, etc. ${ }^{11}$ These interactions are influenced by the broken symmetry at surfaces and interfaces in strongly correlated thin films. ${ }^{50,105}$ Taking advantage of quasiparticle dynamics and interactions is at the heart of multiple technologies, including transistors, solar cells, photonics, and spintronics. A critical gap in our understanding of these interactions is the lack of information we have about the aforementioned interactions at surface and interfaces of the solids, as well as the ultra-thin regime, which are very different than their bulk counterparts. Like many surface and interface properties, the quasiparticles' dynamics and interactions are likely to be very different than their bulk counterparts, having potentially dramatic consequences on device performance. One of the most common quasiparticle interactions is the absorption of the ultrafast laser pulse energy at the surface layers of the solid, and converting that energy to coherent lattice vibrations (phonons). These vibrations can be assumed as a strain wave propagating longitudinally in the bulk, or transversely on the surface and interfaces of the solid with the speed of sound. These acoustic waves are utilized for electro-acoustic filters, mobile phones, and non-destructive material characterization, especially in biomaterials where the electromagnetic waves can be destructive. ${ }^{106,107}$ Moreover, acoustic waves are the main reason for device heating in thin-film devices and different methods of engineering them will enhance devices' performances.

Surface sensitive time-resolved optical measurements, such as second-harmonic generation, is one of the methods to study the quasiparticle interactions at the surface and interface. ${ }^{95}$ However, SHG is surface sensitive only when the bulk symmetry is centrosymmetric, which results in no second harmonic signal. On the other hand, transient reflectivity measurements, using an ultrafast optical setup, can provide significant information about the quasiparticle interactions in the solid, however, this method is not specifically a surface-sensitive method. Generally, TR signal consists of a decay dynamic due to relaxation of charge carriers to lower energy levels, superimposed by multiple sinusoidal quasiparticle interactions (if they exist), such as phonons, magnons, surface plasmon polaritons, etc. ${ }^{108-111}$

More specifically, when a strong laser pulse heats the surface of the thin film, a strain pulse will be generated and start traveling longitudinally in the thin film. Since the strain wave is traveling longitudinally in the thin film, the probe pulse reflected from the surface of the film can interfere 
with the probe pulse reflected from the strain wave. Therefore, the movement of the strain wave which travels with the respective sound velocity of the thin film results in sinusoidal oscillations superimposed on the TR signal. Using this superimposed sinusoidal signal, one can obtain information regarding the sound velocity as well as heat propagation in the thin film.

Windowed Fourier transform

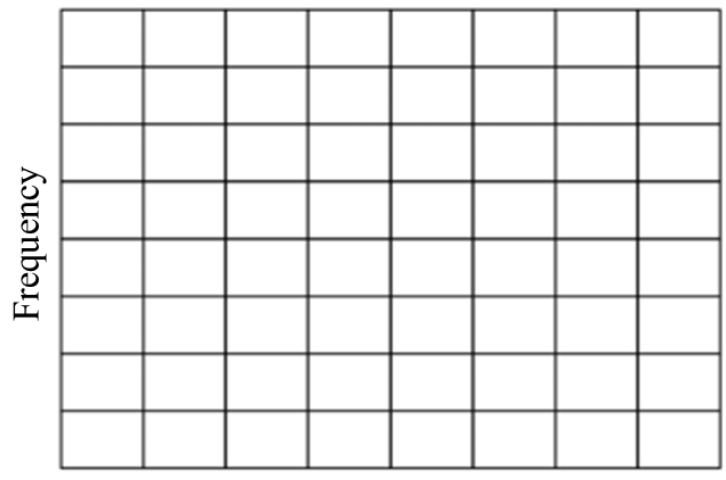

Time
Wavelet transform

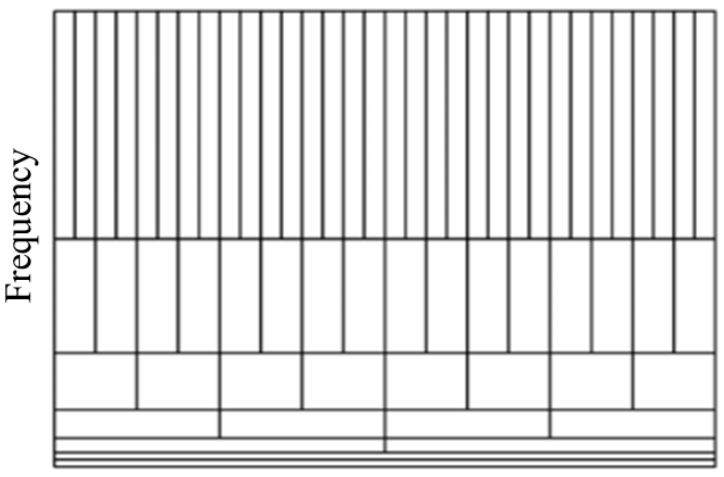

Time

Figure 28. Sampling grid size for (a) Fourier transform and (b) Wavelet transform.

This sinusoidal behavior in TR measurements is commonly analyzed by Fourier transforms or fitting algorithms. ${ }^{108-111}$ However, these traditional methods will not provide all the information possible to extract from the data, especially the abrupt changes happening at the surface and interfaces. Moreover, in films with a thickness of a few nanometers even the bulk related phenomena happen and damp out quickly, which results in the failure of traditional methods to obtain information. The reason for this problem is that Fourier transforms are defined as the sum of sine waves that are not localized in time or space. Although, windowed Fourier transforms (WFT) have been introduced to study the signal in the frequency-time domain, they fail to function in time scales much less or much higher than the window's width, since it introduces a scale into the analysis. ${ }^{12}$ On the other hand, wavelet transforms, which use scalable localized wavelets in time and space as the window function, are perfect tools to analyze abrupt changes in the signals. ${ }^{113}$ Figure 28 presents the difference between the sampling grid size of the windowed Fourier transform and wavelet analysis. While windowed Fourier transform uses uniform sampling grid size, wavelet transform uses dyadic sampling grid size. Wavelet analysis has been commonly used for signal analysis ${ }^{114}$ and image processing. ${ }^{115}$ While there are some studies regarding the application of wavelet analysis in materials science, ${ }^{116-119}$ their applications in TR measurements of ultra-thin film heterostructures have not been well studied yet. In this dissertation, wavelet analysis has been investigated and applied to the sinusoidal signal to extract information related to different vibrational modes, especially at the surface and interfaces which cause abrupt changes in the TR 
signal. We show that the choice of wavelet can extract different information from the same TR signal. In the case of a low noise TR signal, this method will provide more information about the interaction between different vibrational modes in ultra-thin films, compare to the conventional analyzing method.

\subsection{Wavelet Analysis}

In signal processing, information about the signal in the time-frequency domain is a matter of great importance. The first proposed approach for this purpose was WFT, which uses a weight or window function, $g(t)$, to localize the signal in time. Thus, for a real signal of $W(t)$, WFT is defined as: ${ }^{112}$

$$
\tilde{W}(\omega, T)=\int_{-\infty}^{+\infty} e^{-2 \pi i \omega T} \bar{g}(t-T) W(t) d T
$$

Equation 10

By defining $g_{\omega, T}(t)=e^{-2 \pi i \omega T} \bar{g}(t-T)$, WFT can be expressed as the inner product of $g_{\omega, T}$ and $W$. Any feature in WFT is under-localized for the times smaller and over-localized for the times much higher than the width of the window ${ }^{112}$. In other words, WFT fails to isolate the location of each frequency, if the frequency occurrence time is much smaller or much higher than the width of the window.

To solve the scaling problem, wavelet transforms to take advantage of a fully scalable modulated window as an alternative windowing approach in WFT. The window function in the case of wavelet analysis is called the mother wavelet, $\psi(t)$. The scalable modulated form of the window is called daughter wavelet, defined as: ${ }^{112}$

$$
\psi_{s, \tau}(t)=s^{-(1 / 2)} \psi\left(\frac{t-\tau}{s}\right)
$$

With $s$ and $\tau$ defined as the scale and translation factor, respectively. Continuous wavelet transforms are then given as: $:^{112}$

$$
W(\tau, s)=\int_{-\infty}^{+\infty} W(t) \psi_{s, \tau}^{*}(t) d t
$$

Equation 12

Here, $\psi_{S, \tau}^{*}(t)$ is the complex conjugate of the daughter wavelet. By applying a collection of daughter wavelets with different scale and translation factors, the result will be a time-dependent depiction of oscillation frequencies in the signal. Thus, as a result of wavelet analysis, one can have 
information about the different oscillatory modes at different times in a transient, or at different positions if the analysis is applied to TR on heterostructures.

In Equation 11, the basis function is not defined. Unlike a Fourier transforms which has trigonometric polynomial basis functions delocalized in time and space, the wavelet transforms are based on different families of mother wavelets which are localized in time and space. Besides being localized in time and space, a function can be a mother wavelet if it has a mean of zero. Different types of mother wavelet families have been proposed to suit different types of data analysis, such as Gabor-Morlet, ${ }^{120}$ Log Gabor, ${ }^{121}$ Bessel,,${ }^{122}$ Gaussian, ${ }^{123}$ Morse wavelet, ${ }^{124}$ Mexican hat, ${ }^{125}$ etc. Since the implementation of different mother wavelets on the same set of data yield different results, the biggest challenge in wavelet analysis is to find the optimum mother wavelet for a specific signal. Mother wavelets are defined by their orthogonality, the number of vanishing moments and regularity. ${ }^{126}$ The number of vanishing moments and regularity are complex mathematical concepts. ${ }^{126}$ In short, the number of vanishing moments is related to the number of terms in the Taylor expansion of the wavelet transforms at $t=0$ that go to zero. Regularity sometimes refers to how differentiable a function is. Generally, to find the optimum mother wavelet, the similarity between the signal and the mother wavelet is considered. ${ }^{127}$ Abrupt changes in the signal are more distinguishable in the derivatives of the signal. Hence, to detect abrupt changes in the signal, the mother wavelet should be sufficiently regular, that is sufficient number of continuous derivatives for the function. While different mother wavelets show regularity, one of the most regular mother wavelets, which is also similar to the sinusoidal signal, is the Morse wavelet, shown in Figure 29. Based on these criteria, the Morse wavelet, which is a super family of wavelets, has been used as the optimum mother wavelet for detection of abrupt changes in the following data. The Morse wavelet, in the frequency domain, is defined as: ${ }^{124}$

$$
\psi_{\beta, \gamma}(\omega)=\int_{-\infty}^{+\infty} \psi \beta, \gamma(t) e^{-i \omega t} d t=U(\omega) a_{\beta, \gamma} \omega^{\beta} e^{-\omega \gamma}, \quad \text { Equation } 13
$$

Where $U$ is a unit step function and $a$ is a normalizing factor. $\beta$ and $\gamma$ are the compactness and symmetry parameters, respectively. The latter two are the main key considerations for the Morse wavelets, because changing $\beta$ and $\gamma$ can manipulate the Morse wavelets and make it suitable for abrupt changes in the signal. By employing different compactness and symmetry parameters, we indicated some suitable Morse wavelets which can be applied to the TR signal to obtain information regarding the surface, interfaces and very short-lived oscillatory modes. The Gabor limit in signal analysis introduces the trade-off between frequency and time. ${ }^{126}$ Thus, if either 
frequency or time is more important for the scientific goal, some sacrifice in the resolution for the other may be required.

Figure 29 is an indication of what information is obtainable by using different wavelets on the same residual of TR data. Here we compare the results of the wavelet analysis using a generalized Morse wavelet with $\gamma=3$ and $\beta=20$ for the left column and $\gamma=60$ and $\beta=2$ for the right column. Figure 29 (a) and Figure 29 (b), present the real and imaginary parts of the wavelet in time, to show the effect of changing $\beta$ and $\gamma$ on the Morse wavelets. Figure 29 (c) and Figure 29 (d) provide the associated frequency-time distribution of the Morse wavelets shown in Figure 29 (a) and Figure 29 (b). The area of concentration in the frequency-time domain [Figure 29 (c) and Figure 29 (d)] is obtained by reconstruction of the signal, based on the resolution of identity formalism, explained in the reference. ${ }^{128}$ These figures [Figure 29 (a)- Figure 29 (d)] show that by decreasing $\beta$ and increasing $\gamma$ the resolution in time increases, while the resolution in frequency decreases. Thus, by changing the wavelet from a symmetrical form to a more time-compact form in the frequency-time domain, one can obtain information regarding the time and position of more abrupt phenomena happening in the signal. The comparison of the two wavelets is shown in Figure 29 (e) and Figure 29 (f). In the latter, the region highlighted with the red ellipse confirms that better time resolution is achieved by the more compact wavelet. In this case, abrupt signatures are seen arising from both the surface and interface.

To perform wavelet analysis in MATLAB, first, the best multi-decay component function has been fitted to the TR signal as shown in Figure 30. After extraction of the fit residual, the oscillatory signal has been analyzed by wavelet transforms. Figure 29 (a)- Figure 29 (d) are plotted using a freely available MATLAB toolbox called JLAB, available at http://www.jmlilly.net. The generalized Morse wavelets [Figure 29 (a) and Figure 29 (b)] are implemented with morsewave, while the frequency-time domain plots [Figure 29 (c) and Figure 29 (d)] are computed in the function morseregion. The MATLAb code resulting in wavelet analysis is provided in the appendix. 

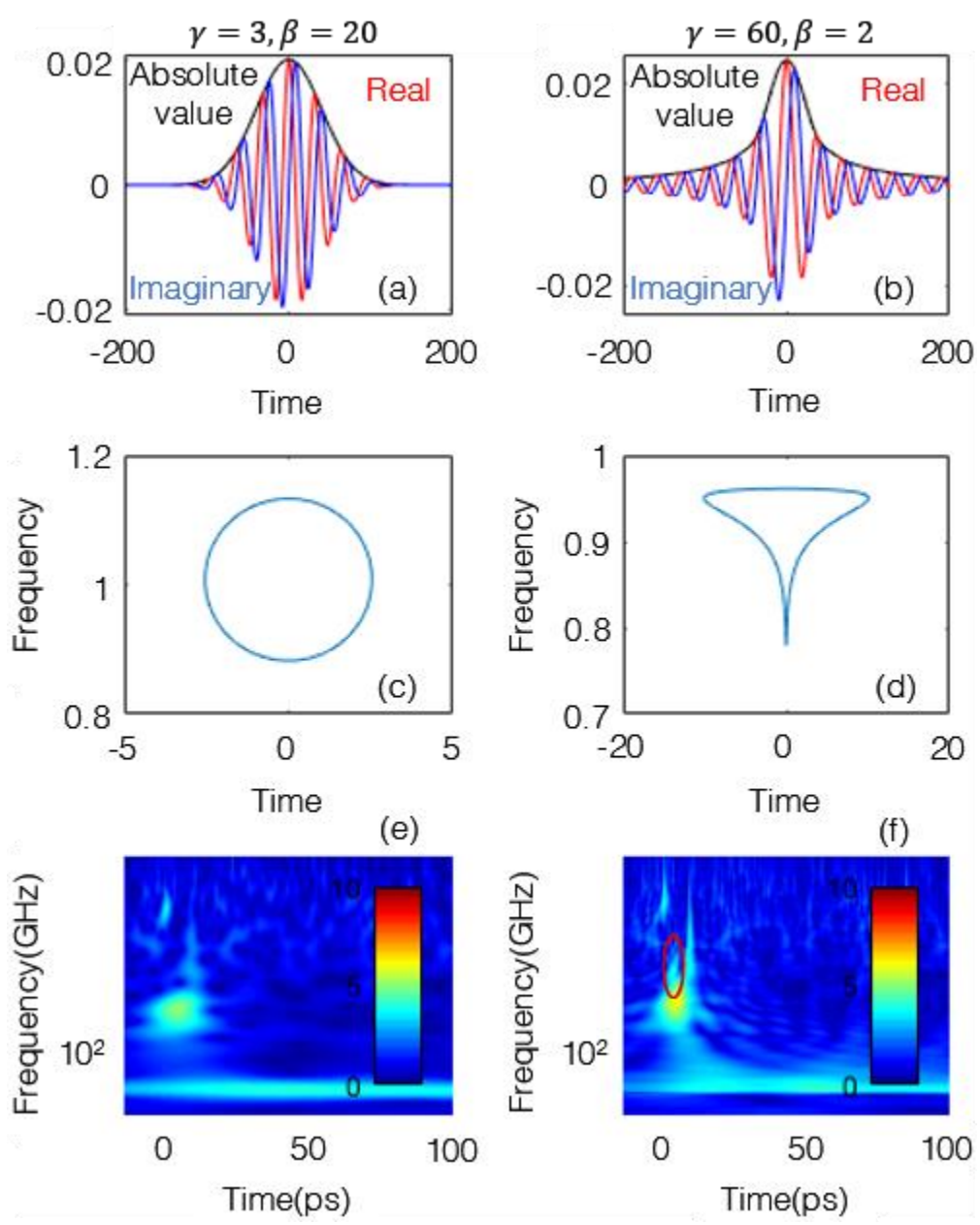

Figure 29. (a) and (b) Real, imaginary and absolute parts of the Morse wavelets. (c) and (d) The frequency-time domain representative of $\mathrm{a}$ and $\mathrm{b}$ with a concentration area of 2 (figures only depict the positive frequencies). (e) and (f) Wavelet transforms of different Morse wavelets on the same residual TR signal. Morse parameters are $\gamma=3, \beta=20$ for a, c, and e and $\gamma=60, \beta=2$ for $\mathrm{b}, \mathrm{d}$, and $\mathrm{f}$.

After defining the Morse wavelet, frequency-time spectra of the signal [Figure 29 (e) and Figure 29 (f), wavelet transforms plots] has been plotted using the $c w t$ function in MATLAB. In the wavelet transforms plot [Figure 29 (e) and Figure 29 (f)], X and Y axes are the time of the occurrence and the frequency of the oscillations, respectively. The amplitude of the oscillations is defined by the color bar.

\subsection{Results}

Measurements are performed on a $\mathrm{La}_{0.7} \mathrm{Sr}_{0.3} \mathrm{MnO}_{3}$ (LSMO) thin film with the thickness of $d=43 \mathrm{~nm}$, grown on a 001 -cut $\mathrm{SrTiO}_{3}(\mathrm{STO})$ substrate in a pulsed laser deposition system at $750^{\circ} \mathrm{C}$ and in oxygen pressure of $100 \mathrm{mTorr}$. Moreover, as a further illustration on a more complicated 
system, the same measurements are performed on the multiferroic heterostructure of $\mathrm{BaTiO}_{3}$ (BTO)/LSMO (ferroelectric/ferromagnetic) grown on an STO (001) substrate. In this case, both BTO and LSMO layers were grown at $750^{\circ} \mathrm{C}$ and in oxygen pressure of 150 mTorr.

As a result of TR measurements performed using a degenerate pump-probe setup, our observations indicate that to utilize wavelet transforms with the resolution of $\Delta f / f \approx 0.5$, where $f$ is the center frequency of an oscillatory mode and $\Delta f$ is its bandwidth, the residual TR signal should have a signal to noise ratio of greater or equal to $83.5 \mathrm{~dB}$. For LSMO thin films which have very weak TR signals, this high signal to noise ratio has been achieved by repeating the scan from a few hours to a few days, corresponding to 50 to 300 scans. Depending on the sample, the strength of the oscillations in the TR signal and the time span of the scan, the number of repetitions can be different.

Figure 30 (a) provides a schematic of the experiment and different oscillatory modes which eventually result in the main sinusoidal reflectivity signal which is due to the interference of reflection from different vibrational modes. The TR result for a $43 \mathrm{~nm}$ LSMO thin film on an STO substrate is shown in Figure 30 (b). ${ }^{63}$ The TR signal consists of a sharp negative rise ${ }^{63}$ due to the band filling effect, ${ }^{129}$ which indicates excitation of the carriers to the higher energy levels. The negative rise in the TR signal is followed by multiple exponential decay processes with different decay time constants $(\tau)$. Electron-phonon recombination $\left(\tau_{\mathrm{e}-\mathrm{p}}<1 \mathrm{ps}\right)$, phonon-assisted spin-lattice recombination $\left(\tau_{\mathrm{p}-\mathrm{s}} \sim 100 \mathrm{ps}\right)$, thermal diffusion and radiative recombination $(\tau>1 \mathrm{~ns})$ are responsible for relaxation of the carriers back to lower energy levels. Moreover, Figure 30 (b) indicates weaker sinusoidal oscillations as the carriers recombine via the multi-exponential decay process. 


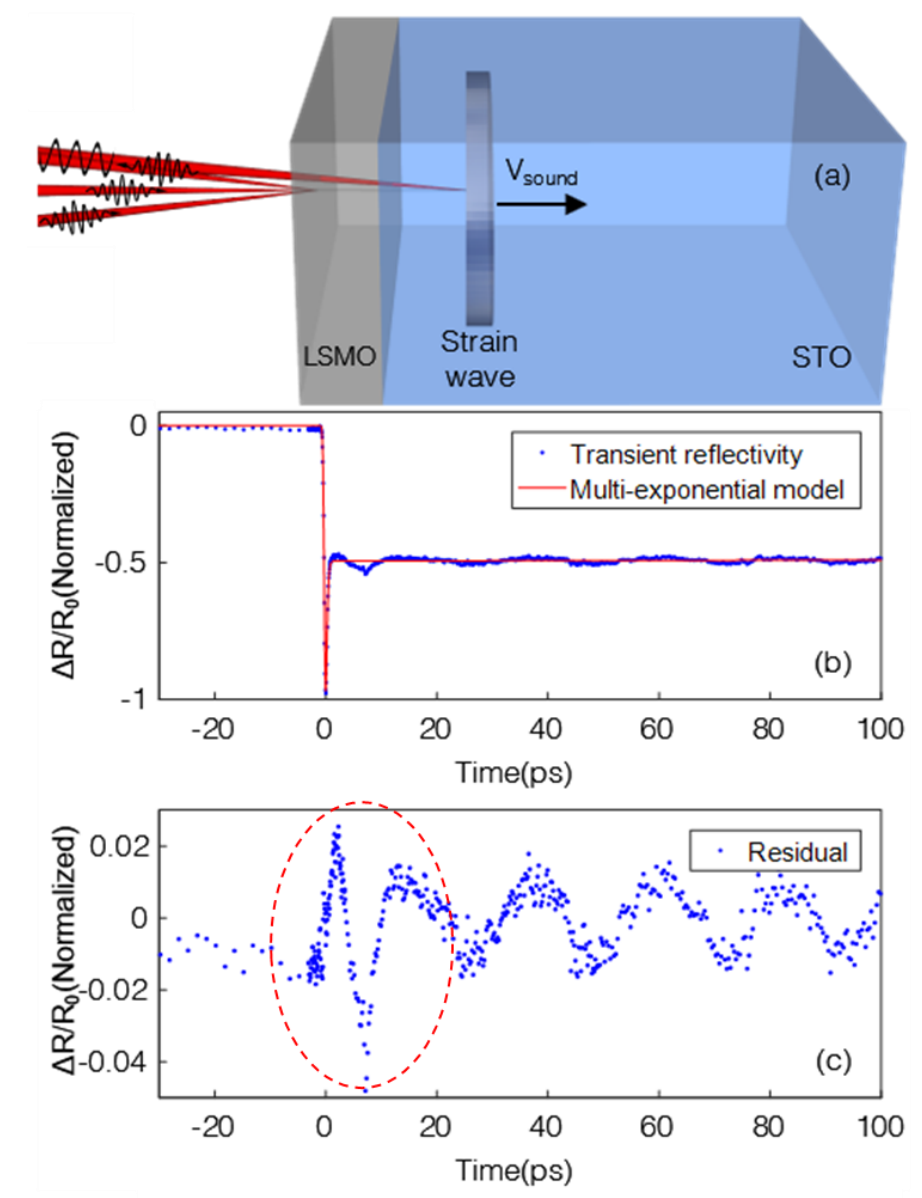

Figure 30. (a) Schematic of the TR experiment and the oscillatory signal in LSMO/STO sample. (b) TR signal for LSMO thin film indicating multiple decay components and oscillatory modes.

(c) Residual of the TR signal after fitting as the input of wavelet analysis.

Figure 30 (c) is the residual of the exponential fit to the TR signal, indicating multiple vibrational modes lasting for hundreds of picoseconds. These vibrational modes are mainly due to carriers' excess energy transferred to the lattice via electron-phonon scattering. Considering the half-metallic behavior of LSMO thin films, LSMO can be used as an optoacoustic metal transducer, ${ }^{130}$ transferring the ultra-short laser pulse to a strain pulse, traveling longitudinally through the film with the speed of sound. Since this strain pulse is traveling in the longitudinal direction, at a specific time, the pulse will hit the interface between the thin film and the substrate, resulting in Brillouin oscillation traveling through the substrate. Due to structural differences between the thin film and the substrate, the secondary strain pulse, which is due to lattice vibrations in the substrate, will have a different frequency compared to that from the thin film. Since the Fourier transforms fail to identify which particular region of the heterostructure is responsible for each oscillatory mode, the wavelet transforms have been used to address this issue. 
As an example, Figure 31 demonstrates some of the physical properties that can be inferred from the result of the wavelet analysis on the fit residual of the TR signal [Figure 30 (b)] from 43 $\mathrm{nm}$ LSMO thin film. In the acquisition of Figure 31 a Morse wavelet with parameters of $\gamma=3$ and $\beta=20$, has been used. The wavelet transforms' plot of the LSMO thin film on an STO substrate indicates a strong, short-lived, vibrational mode starting as the laser pulse hits the surface of the thin film at $100 \mathrm{GHz}$ and a long-lived vibrational mode at $45 \mathrm{GHz}$ in the substrate ${ }^{108}$.

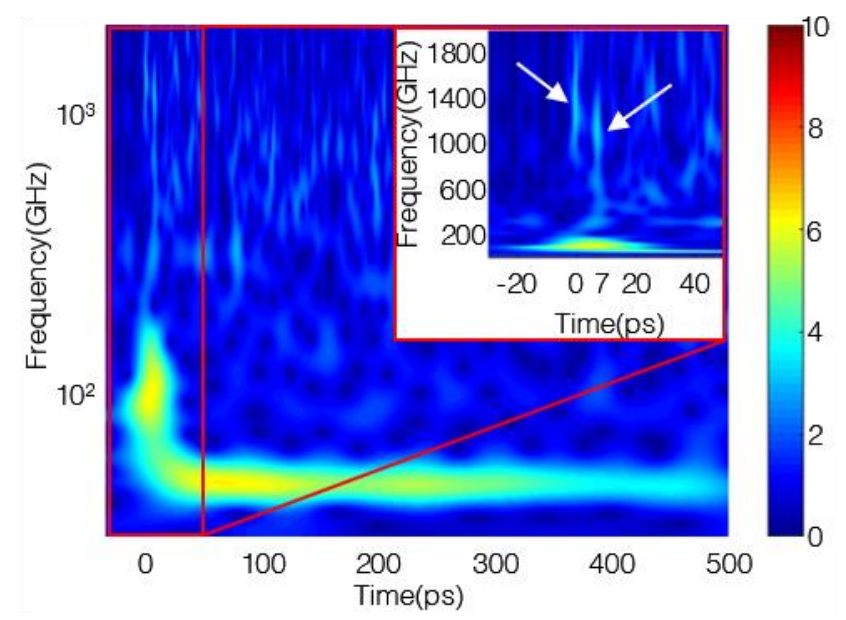

Figure 31. Wavelet analysis on residual of TR signal for $43 \mathrm{~nm}$ LSMO thin film using a Morse wavelet with $\lambda=3$ and $\beta=20$, indicating multiple short and long-lived oscillatory modes (log scale). Inset (linear scale), which is a zoomed-in version of the main figure from -30 ps to $50 \mathrm{ps,}$ is to emphasize the abrupt change at the surface and the interface.

When the strain pulse propagates from one medium to another medium, both the amplitude and the frequency of the oscillations will change. The strain pulse travels through each medium with the respective speed of sound in the medium which can be calculated with:

$$
V_{S}=\frac{f \cdot \lambda_{\text {probe }}}{2 \cdot n \cdot \cos \theta},
$$

Where $f$ is the frequency of the interference patterns and $\lambda_{\text {probe }}, \theta$ and $n$ are the wavelength, incident angle of the probe beam and the refractive index of the medium, respectively. Considering the long-lived oscillations at $45 \mathrm{GHz}$, the speed of sound in the substrate (STO with $n=2.34{ }^{130}$ at $\lambda_{\text {probe }}=810 \mathrm{~nm}$ and probe incident angle of $\left.15^{\circ}\right)$ is $8200 \mathrm{~m} / \mathrm{s}$ which is in good agreement with published literature. ${ }^{131}$ This result indicates that the long-lasting oscillations at $45 \mathrm{GHz}$ are the acoustic phonons propagating in the substrate. The refractive index of the LSMO thin film used in this experiment is confirmed to be 2.3 by ellipsometry. By analogy, the sound velocity for the 
LSMO thin film with a thickness of $43 \mathrm{~nm}$ from the short-lived oscillation at around $100 \mathrm{GHz}$, can be calculated as $\approx 18000 \mathrm{~m} / \mathrm{s}$, which has not been reported for LSMO ultra-thin films.

In the wavelet analysis of the TR measurements of heterostructures, one should not confuse the long-lived oscillatory modes in the thin film with the modes from the substrate. One method to examine whether the oscillatory mode is long-lived or from the substrate is to check the abrupt oscillatory modes, in the time domain, which happen at specific times. The inset of Figure 31 shows a reduced time range of -30 to $50 \mathrm{ps}$, emphasizing two vibrational modes that broaden in frequency, and occurring at around 0 and 7 ps. These modes are examples of abrupt changes in the signal, which is the main advantage of using Morse wavelet analysis. These two modes can be due to higher energy oscillatory modes such as surface and interface lattice vibrations, high energy shortlived magnons, or echoes of the Brillouin oscillations from surface or interface. These echoes from the interface can be observed in delay times equal to multiples of $t_{e c h o}=2 d / V_{S},{ }^{132}$ where $\mathrm{d}$ is the thickness of the thin film. Considering $V_{S} \approx 18000 \mathrm{~m} / \mathrm{s}$ and $t_{e c h o} \approx 7 p s$ for the LSMO sample used in this experiment, this will correspond to $63 \mathrm{~nm}$ of LSMO thin film, indicating that these two modes are related to the surface and interface. Here, the difference in the actual $(43 \mathrm{~nm})$ and calculated $(63 \mathrm{~nm})$ thickness is due to reduced accuracy when $\lambda_{\text {Phonon }} \geq d$. Higher energy modes occurring at random times are likely due to the noise in the signal which can be reduced with more advanced optical techniques such as asynchronous operation and frequency combs.

Figure 32 shows wavelet analysis on the TR signal of the BTO/LSMO/STO heterostructure. Since BTO/LSMO is a multiferroic heterostructure (ferroelectric/ferromagnetic), it is expected to show multiple oscillatory modes in the same energy ranges, which are not trivial to decouple. 


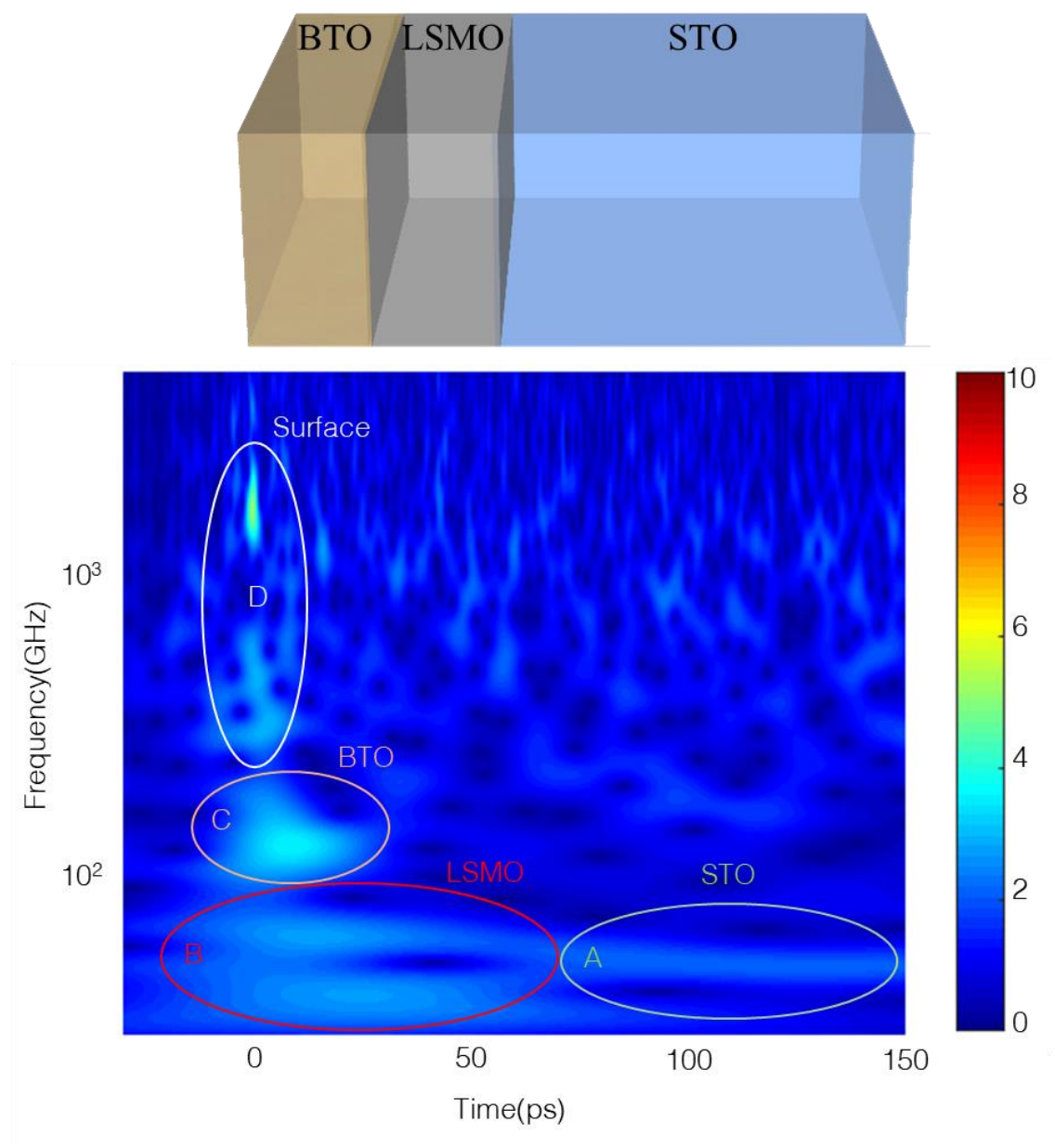

Figure 32. Wavelet analysis of a more complicated system of multiferroic heterostructure (BTO/LSMO/STO), indicating the capability of distinguishing between different oscillatory modes with very close energy ranges.

Indeed, six oscillatory modes are observed in Figure 32. As previously discussed, area A from 70 to $150 \mathrm{ps}$, includes acoustic phonon modes in bulk STO at a frequency of around $45 \mathrm{GHz} .{ }^{133}$ Area B indicates split oscillatory modes between 0 to 70 ps with very close energy ranges of 20 and $60 \mathrm{GHz}$. These two modes are attributed to acoustic phonons and magnons in the ferromagnetic LSMO layer. The oscillatory mode in area $\mathrm{C}$ from 0 to $25 \mathrm{ps}$ at a frequency of $120 \mathrm{GHz}$ occurring in the top layer of the heterostructure and is attributed to acoustic phonons in the BTO layer. Moreover, the short-lived modes in area $\mathrm{D}$, at around $800 \mathrm{GHz}$ and $1.3 \mathrm{THz}$, are attributed to surface/interface phenomena (such as surface phonons and surface plasmons). To distinguish each mode, and provide an accurate physical reason, more measurements in different temperatures and applied magnetic fields are required. 

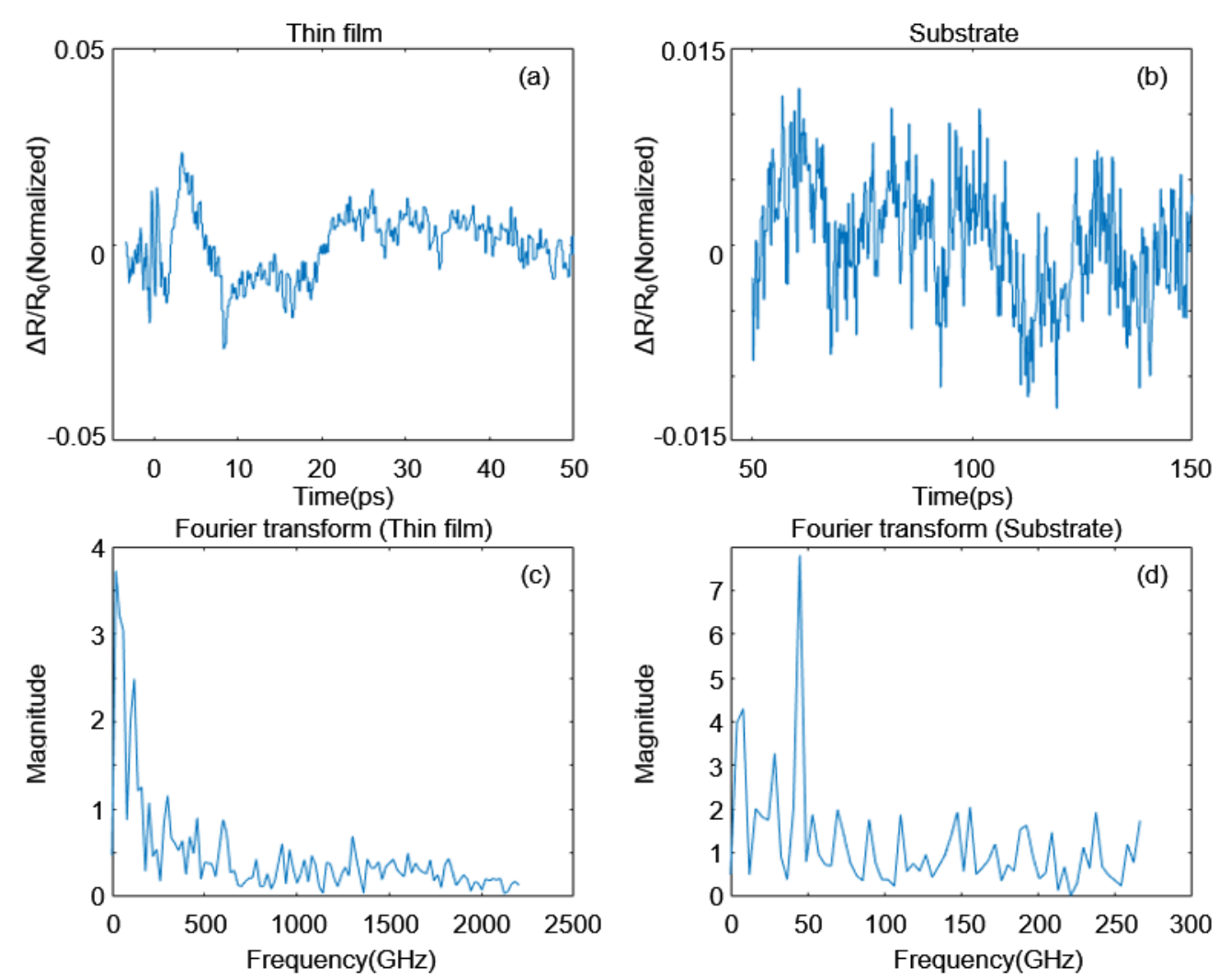

Figure 33. Residual TR signal for BTO/LSMO layers (a) and STO (b). Fourier transforms for BTO/LSMO layers (c) and STO (d).

Comparison with Fourier transforms, to further illustrate the strength of the Morse wavelet analysis is shown in Figure 33. To help improve the results from the Fourier transform, the data has been divided into two parts. The first part (a-c, $\mathrm{t}<50 \mathrm{ps}$ ) indicates the part of the signal which is dominantly affected by the BTO and LSMO layers, however, the second part (b-d, t $>50 \mathrm{ps)}$ is mainly from the substrate (STO). Without this separation, the result is mainly dominated by the substrate section and no information from the thin film section is achievable. Figure 33 (d) indicates the information achieved using the Fourier transforms for the substrate section is comparable to the one achieved with the wavelet analysis. However, Figure 33 (c) indicates when the focus is on the heterostructure thin film, the Fourier transforms are not as informative as the wavelet analysis in Figure 32. 


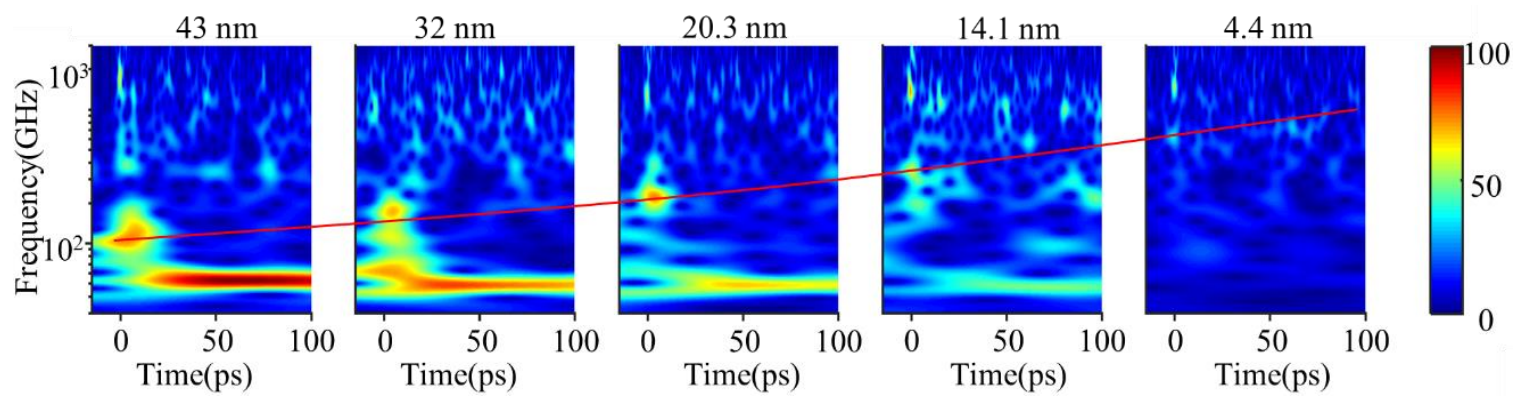

Figure 34. Oscillatory modes due to strain wave propagation for LSMO thin films with different thicknesses.

Since wavelet analysis is capable of identifying very fast vishing oscillations, it can be used to identify the transient strain wave propagation in thin films with respect to their thickness, even if the thickness variation is on the order of a few unit cells. Moreover, since wavelet analysis provides the frequency of the oscillations in the time domain, and because that can be translated into the film thickness, wavelet analysis can inform regarding the energy (heat) transfer between the thin film and the substrate. Generally, in transient strain propagation, if the film's thickness is high, the strain wave propagates with the sound velocity longitudinally in the thin film and eventually transfers into the substrate. However, if the film thickness is very low, a Fabry-Perot resonator forms in the thin film. As a result, the resonator's frequency is expected to increase as the film thickness decreases and no significant energy transfer to the substrate is expected to happen.

Figure 34 presents wavelet analysis results for LSMO thin films grown on STO substrate with different film thicknesses. Although the films' thicknesses are in the range that Fabry-Perot resonator is expected, it can be observed from Figure 34 that a mixture of Fabry-Perot resonator and strain wave propagation exist in LSMO ultra-thin films. Figure 34 indicates that by decreasing the film thickness, the sound velocity in the thin film increases, which is a characteristic of FabryPerot resonators. However, energy transfer between the thin film and the substrate is also observable in LSMO thin films, which is a characteristic of strain wave propagation. The energy transfer decreases as the film thickness decreases and eventually, no energy transfer is observed for LSMO thin film as thin as $4.4 \mathrm{~nm}$. It should be noted again that all this information is achievable using wavelet analysis being used for the first time in this dissertation, and Fourier transform could not resolve all this information.

\subsection{Chapter Summary}

In conclusion, wavelet analysis has been applied to the residual of transient reflectivity signals obtained from ultra-thin films. Implementation of the Morse wavelets, with a variety of 
compactness and symmetry parameters, favors extraction of information in time, position and frequency domains of the oscillations. This implementation illustrates that this technique is a powerful method for analyzing the residual of the transient reflectivity signal.

We suggest this technique to be used on the residual of exponential fits to pump-probe data, when multiple oscillatory modes with the same energy ranges are expected in the heterostructure, as well as studying of abrupt changes or fast decaying oscillatory modes in ultra-thin films.

Using wavelet analysis on the TR signal of LSMO thin films we were able to illustrate a combination of strain wave propagation with significant energy transfer to the substrate and FabryPerot resonator with increasing speed as the film thickness decreases.

Some future applications of this technique include the study of acoustic surface and bulk phonons and magnons, magnon-phonon coupling, and surface plasmon polaritons in ultra-thin films. Advanced laser schemes including asynchronous operation ${ }^{134}$ and frequency combs ${ }^{135}$ can further improve the achievable information from this technique. Moreover, with the emergence of pulsed X-Ray sources found at synchrotrons and free-electron lasers, study of the modes with higher energies such as short-lived THz magnons is conceivable. ${ }^{136}$ 


\section{Chapter 6. Conclusion}

\subsection{Summary of the Dissertation}

In this dissertation, some of the quasiparticle interactions in complex oxides, in extreme conditions of the ultra-thin region, have been investigated. The main challenge facing optical experiments in ultra-thin regime is insufficient material to absorb the light, therefore not enough information is understood in this regime. Understanding the optical properties in ultra-thin regime will help the industry to reduce the cost of devices by using less materials, as well as increasing the efficiency of many devices such as solar cells, ultrafast switches, spintronic devices, etc. Static optical properties of LSMO ultra-thin films, using spectroscopic ellipsometry exhibits optical transitions of LSMO's carriers to Ti's empty orbitals from the substrate at the interface. These new transitions result in new absorption peaks which appear only in the ultra-thin regime and are corroborated using DFT calculations.

Performing the pump-probe experiment on the thinnest film ever reported to the best of our knowledge, indicated the ultrafast dynamics of surface recombination. Our observations indicate that surface recombination, that usually is understood as detrimental for devices, can be the key feature for some specific devices. More specifically, in the case of ultra-thin LSMO films, surface recombination results in almost a complete carrier's energy loss in less than a hundred picosecond which suggests these ultra-thin films to be useful in ultrafast spintronic switches. Moreover, transient reflectivity for oxygen stoichiometric LSMO thin films with different oxygen content exhibit the effect of oxygen content on phonon-assisted relaxation mechanisms. Based on our observations, increasing the oxygen content in the oxide will increase the contribution of phononassisted relaxation mechanisms in the oxides. Additionally, a circular pump-probe experiment on LSMO thin films was used to address the photoinduced spin polarization of the system. Our results, which are corroborated with DFT calculation, indicate two spin depolarization mechanisms with the lifetimes of a few hundred femtoseconds and a few hundred picoseconds, respectively. Moreover, our observations indicate drastic photoinduced features in the slower spin depolarization mechanism.

Lastly, wavelet analysis was applied, for the first time, to the best of our knowledge, to address transient strain waves in ultra-thin and multilayer films. Transient strain waves are the basis of sensing devices that are being used on the materials that are prone to be destroyed under the influence of electromagnetic waves, such as biological tissues. Based on wavelet analysis, and more specifically, the Morse wavelet, we were able to extract all the information from interference 
patterns superimposed on the TR signal. Hence, we suggest this analyzing method based on wavelet transforms to be used to address the sound velocity, heat propagation and energy transfer in ultrathin films and multilayer heterostructure with the resolution of layer by layer. Our findings indicate strain propagation longitudinally in LSMO ultra-thin films which become faster as the thickness of the film decreases. Moreover, using the new technique shear strain waves on the surface and the interface of the films were observable.

\subsection{Future Research}

While some of the quasiparticle interactions in LSMO complex oxides have been addressed in this dissertation, there is still more to learn from these complex oxides and their interactions. Our initial findings predict a promising future for LSMO ultra-thin films in spintronics devices. However, more studies can be useful to find the unique applications that these ultra-thin films can have in the industry. Mainly focusing on the photoinduced spin polarization is of great importance. Performing non-degenerate pump-probe experiment will specifically focus on each particular transition mentioned in chapter three. Therefore, the energy range in which surface recombination is dominant can be identified. Moreover, non-degenerate experiment will drastically reduce the noise to signal ratio caused by the scattered pump beam. Therefore, circular pump-probe can be performed on lower thicknesses. circular pump-probe experiments on LSMO ultra-thin films with different thicknesses and oxygen content can provide more information on their spintronic applications. Specifically, if understood well, the femtosecond scale drop in spin polarization can be used for ultrafast switches. The next idea worth pursuing is the coupled dynamics in the multiferroic heterostructures. Multiferroic heterostructures such as ferroelectric(BTO)/ferromagnetic(LSMO) systems (Figure 32) are prone to exhibit large controllable coupling between their ferroelectric and ferromagnetic features at the interface. The experiment to study this coupling can be designed as a second harmonic generation pump-probe spectroscopy. By changing the magnetic ordering in the ferromagnetic layer (LSMO) using an external magnetic field or circular excitation pulse, any change in the TRSHG signal should be attributed to the coupling between the ferroelectric and ferromagnetic layers. Therefore, this idea can be used to understand the electric properties by changing magnetic properties or vice versa. 


\section{References}

1. Nomerovannaya, L. V., Makhnëv, A. A. \& Rumyantsev, A. Y. Evolution of the optical properties of single-crystal $\mathrm{La}_{1-\mathrm{x}} \mathrm{SrxMnO}_{3}$. Phys. Solid State 41, 1322-1326 (1999).

2. Liu, H. L. et al. Thickness-dependent optical properties of $\mathrm{La}_{0.7} \mathrm{Sr}_{0.3} \mathrm{MnO}_{3}$ thin films. Journal of Applied Physics 97, 113528 (2005).

3. Martino, M. et al. $\mathrm{La}_{0.7} \mathrm{Sr}_{0.3} \mathrm{MnO}_{3}$ thin films deposited by pulsed laser ablation for spintronic applications. Phys. Status Solidi A 208, 1817-1820 (2011).

4. Cesaria, M., Caricato, A. P., Leggieri, G., Martino, M. \& Maruccio, G. Optical response of oxygen deficient $\mathrm{La}_{0.7} \mathrm{Sr}_{0.3} \mathrm{MnO}_{3}$ thin films deposited by pulsed laser deposition. Thin Solid Films 545, 592-600 (2013).

5. Lobad, A. I., Averitt, R. D., Kwon, C. \& Taylor, A. J. Spin-lattice interaction in colossal magnetoresistance manganites. Appl. Phys. Lett. 77, 4025-4027 (2000).

6. Parlato, L. et al. Time-resolved optical response of all-oxide $\mathrm{YBa}_{2} \mathrm{Cu}_{3} \mathrm{O}_{7} / \mathrm{La}_{0.7} \mathrm{Sr}_{0.3} \mathrm{MnO}_{3}$ proximitized bilayers. Phys. Rev. B 87, 134514 (2013).

7. Müller, G. M. et al. Spin polarization in half-metals probed by femtosecond spin excitation. Nature Materials 8, 56 (2009).

8. Ren, Y. H., Trigo, M., Merlin, R., Adyam, V. \& Li, Q. Generation and detection of coherent longitudinal acoustic phonons in the $\mathrm{La}_{0.67} \mathrm{Sr}_{0.33} \mathrm{MnO}_{3}$ thin films by femtosecond light pulses. Appl. Phys. Lett. 90, 251918 (2007).

9. Vasa, P. Strong Light-Matter Interaction. in digital Encyclopedia of Applied Physics 1-30 (American Cancer Society, 2019). doi:10.1002/3527600434.eap828.

10. Lienau, C., Noginov, M. A. \& Lončar, M. Light-matter interactions at the nanoscale. J. Opt. 16, 110201 (2014).

11. Venema, L. et al. The quasiparticle zoo. Nature Physics 12, 1085-1089 (2016).

12. Snoke, D. W. Solid State Physics: Essential Concepts. Cambridge Core (2020) doi:10.1017/9781108123815. 
13. Wang, G. et al. Colloquium: Excitons in atomically thin transition metal dichalcogenides. Rev. Mod. Phys. 90, 021001 (2018).

14. YU, P. \& Cardona, M. Fundamentals of Semiconductors: Physics and Materials Properties. (Springer-Verlag, 2010). doi:10.1007/978-3-642-00710-1.

15. Gandolfi, M. et al. Emergent ultrafast phenomena in correlated oxides and heterostructures. Physica Scripta 92, 034004 (2017).

16. Shah, J. Ultrafast Spectroscopy of Semiconductors and Semiconductor Nanostructures. (Springer-Verlag, 1999). doi:10.1007/978-3-662-03770-6.

17. Jellison, G. E., Boatner, L. A., Lowndes, D. H., McKee, R. A. \& Godbole, M. Optical functions of transparent thin films of $\mathrm{SrTiO}_{3}, \mathrm{BaTiO}_{3}$, and $\mathrm{SiOx}$ determined by spectroscopic ellipsometry. Appl. Opt., AO 33, 6053-6058 (1994).

18. Smolin, S. Y., Scafetta, M. D., Guglietta, G. W., Baxter, J. B. \& May, S. J. Ultrafast transient reflectance of epitaxial semiconducting perovskite thin films. Applied Physics Letters 105, 022103 (2014).

19. Sheu, Y. M. et al. Ultrafast carrier dynamics and radiative recombination in multiferroic BiFeO3. Appl. Phys. Lett. 100, 242904 (2012).

20. Alloul, H. Strongly Correlated Electrons in Solids. Scholarpedia 9, 32067 (2014).

21. Liao, Z. \& Zhang, J. Metal-to-Insulator Transition in Ultra-thin Manganite Heterostructures. Applied Sciences 9, 144 (2019).

22. Haghiri-Gosnet, A.-M. \& Renard, J.-P. CMR manganites: physics, thin films and devices. J. Phys. D: Appl. Phys. 36, R127 (2003).

23. Veis, M. et al. Magneto-optic spectroscopy of $\mathrm{La}_{2 / 3} \mathrm{Sr}_{1 / 3} \mathrm{MnO}_{3}$ films on $\mathrm{SrTiO}_{3}\left(\begin{array}{lll}1 & 0 & 0\end{array}\right)$ and (1 1 0) substrates. J. Phys. D: Appl. Phys. 42, 195002 (2009).

24. Damjanovic, D. Ferroelectric, dielectric and piezoelectric properties of ferroelectric thin films and ceramics. Rep. Prog. Phys. 61, 1267 (1998).

25. Jonker, G. H. \& Van Santen, J. H. Ferromagnetic compounds of manganese with perovskite 
structure. Physica 16, 337-349 (1950).

26. Pradhan, A. K. et al. Magnetic properties of $\mathrm{La}_{0.6} \mathrm{Sr}_{0.4} \mathrm{MnO}_{3}$ thin films on $\mathrm{SrTiO}_{3}$ and buffered Si substrates with varying thickness. Journal of Applied Physics 103, 023914 (2008).

27. Haghiri-Gosnet, A. M. et al. Microstructure and magnetic properties of strained La0.7Sr0.3MnO3 thin films. Journal of Applied Physics 88, 4257-4264 (2000).

28. Guo, E.-J. et al. Orientation Control of Interfacial Magnetism at $\mathrm{La}_{0.67} \mathrm{Sr}_{0.33} \mathrm{MnO}_{3} / \mathrm{SrTiO}_{3}$ Interfaces. ACS Applied Materials \& Interfaces 9, 19307-19312 (2017).

29. Španková, M. et al. Characterization of epitaxial LSMO thin films with high Curie temperature prepared on different substrates. Vacuum 126, 24-28 (2016).

30. Smolin, S. Y. et al. Static and Dynamic Optical Properties of $\mathrm{La}_{1-\mathrm{x}} \mathrm{Sr}_{\mathrm{x}} \mathrm{FeO}_{3-\delta}$ : The Effects of A-Site and Oxygen Stoichiometry. Chem. Mater. 28, 97-105 (2016).

31. Solignac, A. et al. Magnetic tunnels junctions for all-oxide spin valves devices. Journal of Physics: Conference Series 303, 012059 (2011).

32. Moreno, C. et al. Reversible Resistive Switching and Multilevel Recording in $\mathrm{La}_{0.7} \mathrm{Sr}_{0.3} \mathrm{MnO}_{3}$ Thin Films for Low Cost Nonvolatile Memories. Nano Lett. 10, 3828-3835 (2010).

33. Alldredge, L. M. B., Chopdekar, R. V., Nelson-Cheeseman, B. B. \& Suzuki, Y. Spinpolarized conduction in oxide magnetic tunnel junctions with magnetic and nonmagnetic insulating barrier layers. Appl. Phys. Lett. 89, 182504 (2006).

34. Kim, B. et al. Reentrant insulating state in ultra-thin manganite films. Appl. Phys. Lett. 99, 092513 (2011).

35. Tebano, A. et al. Evidence of orbital reconstruction at interfaces in ultra-thin $\mathrm{La}_{0.67} \mathrm{Sr}_{0.33} \mathrm{MnO}_{3}$ films. Phys. Rev. Lett. 100, 137401 (2008).

36. Lepetit, M.-B., Mercey, B. \& Simon, C. Interface Effects in Perovskite Thin Films. Phys. Rev. Lett. 108, 087202 (2012).

37. Peng, R. et al. Tuning the dead-layer behavior of $\mathrm{La}_{0.67} \mathrm{Sr}_{0.33} \mathrm{MnO}_{3} / \mathrm{SrTiO}_{3}$ via interfacial engineering. Appl. Phys. Lett. 104, 081606 (2014). 
38. Liao, Z. et al. Origin of the metal-insulator transition in ultra-thin films of $\mathrm{La}_{2 / 3} \mathrm{Sr}_{1 / 3} \mathrm{MnO}_{3}$. Phys. Rev. B 92, 125123 (2015).

39. Huijben, M. et al. Critical thickness and orbital ordering in ultra-thin $\mathrm{La}_{0.7} \mathrm{Sr}_{0.3} \mathrm{MnO}_{3}$ films. Phys. Rev. B 78, 094413 (2008).

40. Hemberger, J. et al. Structural, magnetic, and electrical properties of single-crystalline $\mathrm{La}_{1-\mathrm{x}} \mathrm{Sr}_{\mathrm{x}} \mathrm{MnO}_{3}(0.4<\mathrm{x}<0.85)$. Phys. Rev. B 66, 094410 (2002).

41. Petrov, A. Y. et al. Room temperature metal-insulator transition in as grown $\left(\mathrm{La}_{1-\mathrm{x}} \mathrm{Sr}_{\mathrm{x}}\right)_{\mathrm{y}} \mathrm{MnO} 3$ thin films deposited by molecular beam epitaxy. Eur. Phys. J. B 40, 11-17 (2004).

42. Schwartz, R. W. Chemical Solution Deposition of Perovskite Thin Films. Chem. Mater. 9, 2325-2340 (1997).

43. NuRgalieV, T., TOPAL, U., BlagoeV, B. \& MATEEV, E. Magnetic Properties of LCMO/LSMO Thin Films on LAO and ALO Substrates. J. supercond. nov. magn 25, 24952498 (2012).

44. Pulsed laser deposition of thin films: applications-led growth of functional materials. (WileyInterscience, 2007).

45. http://www.germantech.com.cn/.

46. Speakman, S. A. Introduction to X-Ray Powder Diffraction Data Analysis. 25.

47. Björck, M. \& Andersson, G. GenX: an extensible X-ray reflectivity refinement program utilizing differential evolution. J Appl Cryst, J Appl Crystallogr 40, 1174-1178 (2007).

48. Parratt, L. G. Surface Studies of Solids by Total Reflection of X-Rays. Phys. Rev. 95, 359369 (1954).

49. Trappen, R. B. Depth dependent atomic valence determination in $\mathrm{La}_{0.7} \mathrm{Sr}_{0.3} \mathrm{MnO}_{3}$ thin films using synchrotron techniques. 67.

50. Pesquera, D. et al. Surface symmetry-breaking and strain effects on orbital occupancy in transition metal perovskite epitaxial films. Nature Communications 3, 1189 (2012).

51. Kawasaki, M. et al. Atomic Control of the $\mathrm{SrTiO}_{3}$ Crystal Surface. Science 266, 1540-1542 
(1994).

52. Connell, J. G., Isaac, B. J., Ekanayake, G. B., Strachan, D. R. \& Seo, S. S. A. Preparation of atomically flat $\mathrm{SrTiO}_{3}$ surfaces using a deionized-water leaching and thermal annealing procedure. Appl. Phys. Lett. 101, 251607 (2012).

53. Boschker, H. et al. Optimized fabrication of high quality $\mathrm{La}_{0.67} \mathrm{Sr}_{0.33} \mathrm{MnO}_{3}$ thin films considering all essential characteristics. J. Phys. D: Appl. Phys. 44, 205001 (2011).

54. Ichimiya, A. \& Cohen, P. I. Reflection High-Energy Electron Diffraction. Reflection HighEnergy Electron Diffraction, by Ayahiko Ichimiya and Philip I. Cohen, pp. 366. ISBN 0521453739. Cambridge, UK: Cambridge University Press, December 2004. (2004).

55. Uba, L., Uba, S., Germash, L. P., Bekenov, L. V. \& Antonov, V. N. Electronic structure and magneto-optical spectra of $\mathrm{La}_{x} \mathrm{Sr}_{1-\mathrm{x}} \mathrm{MnO}_{3}$ perovskites: Theory and experiment. Physical Review B - Condensed Matter and Materials Physics 85, 1-12 (2012).

56. Tikhii, A. A. et al. An ellipsometric method for determining the optical parameters of thinfilm coatings with a complex structure. Opt. Spectrosc. 119, 268-272 (2015).

57. Gruenewald, J. H., Nichols, J. \& Seo, S. S. A. Pulsed laser deposition with simultaneous in situ real-time monitoring of optical spectroscopic ellipsometry and reflection high-energy electron diffraction. Review of Scientific Instruments 84, 043902 (2013).

58. 6. Spectroscopic Ellipsometry. http://www.kristoflodewijks.be/research/theoreticalbackground/6-spectroscopic-ellipsometry/ (2013).

59. J.A Woolam M-2000U. J.A. Woollam https://www.jawoollam.com/.

60. CompleteEASE. J.A. Woollam https://www.jawoollam.com/ellipsometrysoftware/completeease.

61. Johs, B. \& Hale, J. S. Dielectric function representation by B-splines. phys. stat. sol. (a) 205, 715-719 (2008).

62. Liu, H. L. et al. Magneto-optical properties of $\mathrm{La}_{0.7} \mathrm{Sr}_{0.3} \mathrm{MnO}_{3}$ thin films with perpendicular magnetic anisotropy. Journal of Applied Physics 99, 043908 (2006). 
63. Yousefi Sarraf, S. et al. Surface Recombination in Ultra-Fast Carrier Dynamics of Perovskite Oxide $\mathrm{La}_{0.7} \mathrm{Sr}_{0.3} \mathrm{MnO}_{3}$ Thin Films. ACS Nano (2019) doi:10.1021/acsnano.8b09595.

64. Millis, A. J. Lattice effects in magnetoresistive manganese perovskites. Nature 392, 147-150 (1998).

65. Sandomirskii, V. Quantum size effect in a semimetal film. Sov. Phys. JETP 25, 101 (1967).

66. Jahn, H. A., Teller, E. \& Donnan, F. G. Stability of polyatomic molecules in degenerate electronic states - I-Orbital degeneracy. Proceedings of the Royal Society of London. Series A - Mathematical and Physical Sciences 161, 220-235 (1937).

67. Okimoto, Y., Katsufuji, T., Ishikawa, T., Arima, T. \& Tokura, Y. Variation of electronic structure in $\mathrm{La}_{1}{ }_{\mathrm{x}} \mathrm{Sr}_{\mathrm{x}} \mathrm{MnO}_{3}(0<\mathrm{x}<0.3)$ as investigated by optical conductivity spectra. Phys. Rev. B 55, 4206-4214 (1997).

68. Prellier, W., Lecoeur, P. \& Mercey, B. Colossal-magnetoresistive manganite thin films. J. Phys.: Condens. Matter 13, R915-R944 (2001).

69. Quijada, M. et al. Optical conductivity of manganites: Crossover from Jahn-Teller small polaron to coherent transport in the ferromagnetic state. Phys. Rev. B 58, 16093-16102 (1998).

70. Peng, L., Cai, C., Xu, D. H. \& Liu, Y. Ultrafast optical probes of dynamic behavior in $\mathrm{La}_{0.7} \mathrm{Sr}_{0.3} \mathrm{MnO}_{3} / \mathrm{YBa}_{2} \mathrm{Cu}_{3} \mathrm{O}_{7-\delta} / \mathrm{La}_{0.7} \mathrm{Sr}_{0.3} \mathrm{MnO}_{3}$ heterostructure. Jetp Lett. 99, $27-31$ (2014).

71. Aspnes, D. Recombination at semiconductor surfaces and interfaces. in Surfaces and Interfaces: Physics and Electronics 406-421 (Elsevier, 1983).

72. Wen, X. et al. Role of Surface Recombination in Halide Perovskite Nanoplatelets. ACS Applied Materials \& Interfaces 10, 31586-31593 (2018).

73. Adhikari, N. et al. Interfacial Study to Suppress Charge Carrier Recombination for High Efficiency Perovskite Solar Cells. ACS Applied Materials \& Interfaces 7, 26445-26454 (2015).

74. Wu, S., Wen, L., Cheng, G., Zheng, R. \& Wu, X. Surface Morphology-Dependent Photoelectrochemical Properties of One-Dimensional Si Nanostructure Arrays Prepared by 
Chemical Etching. ACS Applied Materials \& Interfaces 5, 4769-4776 (2013).

75. Galli, D. et al. Suppressing the Surface Recombination and Tuning the Open-Circuit Voltage of Polymer/Fullerene Solar Cells by Implementing an Aggregative Ternary Compound. ACS Applied Materials \& Interfaces 10, 28803-28811 (2018).

76. Bennett, B. R., Soref, R. A. \& Alamo, J. A. D. Carrier-induced change in refractive index of InP, GaAs and InGaAsP. IEEE Journal of Quantum Electronics 26, 113-122 (1990).

77. Berera, R., van Grondelle, R. \& Kennis, J. T. M. Ultrafast transient absorption spectroscopy: principles and application to photosynthetic systems. Photosynth Res 101, 105-118 (2009).

78. Senty, T. R. Experimental Techniques for Understanding Light Matter Interactions in Novel Materials. 112.

79. Ma, H. et al. Highly Spin-Polarized Carrier Dynamics and Ultralarge Photoinduced Magnetization in $\mathrm{CH}_{3} \mathrm{NH}_{3} \mathrm{PbI}_{3}$ Perovskite Thin Films. Nano Lett. 15, 1553-1558 (2015).

80. Kresse, G. \& Hafner, J. Ab initio molecular dynamics for liquid metals. Physical Review B 47, 558-561 (1993).

81. Kresse, G. \& Furthmüller, J. Efficient iterative schemes for ab initio total-energy calculations using a plane-wave basis set. Physical Review B - Condensed Matter and Materials Physics 54, 11169-11186 (1996).

82. Gajdoš, M., Hummer, K., Kresse, G., Furthmüller, J. \& Bechstedt, F. Linear optical properties in the projector-augmented wave methodology. Physical Review B - Condensed Matter and Materials Physics 73, 1-9 (2006).

83. Perdew, J. P. et al. Restoring the Density-Gradient Expansion for Exchange in Solids and Surfaces. Phys. Rev. Lett. 100, 136406 (2008).

84. Blöchl, P. E. Projector augmented-wave method. Physical Review B 50, 17953-17979 (1994).

85. Joubert, D. From ultrasoft pseudopotentials to the projector augmented-wave method. Physical Review B - Condensed Matter and Materials Physics 59, 1758-1775 (1999). 
86. Bellaiche, L. \& Vanderbilt, D. Virtual crystal approximation revisited: Application to dielectric and piezoelectric properties of perovskites. Physical Review B - Condensed Matter and Materials Physics 61, 7877-7882 (2000).

87. Pack, J. D. \& Monkhorst, H. J. 'special points for Brillouin-zone integrations'-a reply. Physical Review B 16, 1748-1749 (1977).

88. Liechtenstein, A. I., Anisimov, V. I. \& Zaanen, J. Density-functional theory and strong interactions: Orbital ordering in Mott-Hubbard insulators. Physical Review B 52, 5467-5471 (1995).

89. Trappen, R. et al. Electrostatic potential and valence modulation in $\mathrm{La}_{0.7} \mathrm{Sr}_{0.3} \mathrm{MnO}_{3}$ thin films. Scientific Reports 8, 14313 (2018).

90. Lee, J. H., Delaney, K. T., Bousquet, E., Spaldin, N. A. \& Rabe, K. M. Strong coupling of Jahn-Teller distortion to oxygen-octahedron rotation and functional properties in epitaxially strained orthorhombic LaMnO 3. Physical Review B 88, (2013).

91. S J Hibble et. Related content Local distortions in the colossal magnetoresistive. J. Phys.: Condens. Matter 11, 9221-9238 (1999).

92. Glinka, Y. D. et al. Ultrafast carrier dynamics in thin-films of the topological insulator $\mathrm{Bi}_{2} \mathrm{Se}_{3}$. Appl. Phys. Lett. 103, 151903 (2013).

93. Lee, H.-S. \& Park, H.-H. Band Structure Analysis of $\mathrm{La}_{0.7} \mathrm{Sr}_{0.3} \mathrm{MnO}_{3}$ Perovskite Manganite Using a Synchrotron. Advances in Condensed Matter Physics 2015, e746475 (2015).

94. Dai, Y. M. et al. Ultrafast carrier dynamics in the large-magnetoresistance material $\mathrm{WTe}_{2}$. Phys. Rev. B 92, 161104 (2015).

95. Glinka, Y. D., Babakiray, S., Johnson, T. A., Holcomb, M. B. \& Lederman, D. Nonlinear optical observation of coherent acoustic Dirac plasmons in thin-film topological insulators. Nature Communications 7, 13054 (2016).

96. Sundaram, S. K. \& Mazur, E. Inducing and probing non-thermal transitions in semiconductors using femtosecond laser pulses. Nature Materials 1, 217-224 (2002).

97. Sheu, Y. M., Chien, Y. J., Uher, C., Fahy, S. \& Reis, D. A. Free-carrier relaxation and lattice 
heating in photoexcited bismuth. Phys. Rev. B 87, 075429 (2013).

98. Ikezawa, M. et al. Submillisecond electron spin relaxation in InP quantum dots. Physical Review B 72, (2005).

99. Yoshida, S. et al. Probing ultrafast spin dynamics with optical pump-probe scanning tunnelling microscopy. Nature Nanotechnology 9, 588-593 (2014).

100. Heers, S. Effect of Spin-orbit Scattering on Transport Properties of Low-dimensional Dilute Alloys. (Forschungszentrum Jülich, 2011).

101. Sichert, J. A. et al. Quantum Size Effect in Organometal Halide Perovskite Nanoplatelets. Nano Letters 15, 6521-6527 (2015).

102. $\mathrm{Zu}, \mathrm{F}$. et al. Surface State Density Determines the Energy Level Alignment at Hybrid Perovskite/Electron Acceptors Interfaces. ACS Applied Materials \& Interfaces 9, 4154641552 (2017).

103. Zhang, Z. \& Yates, J. T. Band Bending in Semiconductors: Chemical and Physical Consequences at Surfaces and Interfaces. Chemical Reviews 112, 5520-5551 (2012).

104. Lin, X. E. Laser Pulse Heating. New York 3 (1999).

105. Ogawa, Y. et al. Nonlinear Magneto-Optical Kerr Rotation of an Oxide Superlattice with Artificially Broken Symmetry. Phys. Rev. Lett. 90, 217403 (2003).

106. Ducousso, M. et al. Evaluation of mechanical properties of fixed bone cells with submicrometer thickness by picosecond ultrasonics. Eur. Phys. J. Appl. Phys. 61, 11201 (2013).

107. Rossignol, C. et al. In Vitro picosecond ultrasonics in a single cell. Appl. Phys. Lett. 93, 123901 (2008).

108. Chen, L. Y. et al. Ultrafast photoinduced mechanical strain in epitaxial $\mathrm{BiFeO}_{3}$ thin films. Applied Physics Letters 101, 041902 (2012).

109. Talbayev, D. et al. Detection of Coherent Magnons via Ultrafast Pump-Probe Reflectance Spectroscopy in Multiferroic $\mathrm{Ba}_{0.6} \mathrm{Sr}_{1.4} \mathrm{Zn}_{2} \mathrm{Fe}_{12} \mathrm{O}_{22}$. Physical Review Letters 101, (2008).

110. Doig, K. I. et al. Coherent magnon and acoustic phonon dynamics in tetragonal and rare- 
earth-doped $\mathrm{BiFeO}_{3}$ multiferroic thin films. PHYSICAL REVIEW B 88, (2013).

111. Glinka, Y. D., Babakiray, S., Johnson, T. A., Holcomb, M. B. \& Lederman, D. Acoustic phonon dynamics in thin-films of the topological insulator $\mathrm{Bi}_{2} \mathrm{Se}_{3}$. Journal of Applied Physics 117,165703 (2015).

112. Kaiser, G. A friendly guide to wavelets. (Birkhäuser, 2011).

113. Meyer, F. Wavelets and Applications. Wavelets and Applications 8.

114. Rioul, O. \& Vetterli, M. Wavelets and signal processing. IEEE Signal Processing Magazine 8, 14-38 (1991).

115. Chan, T. F. \& Shen, J. (Jackie). Image Processing and Analysis: Variational, PDE, Wavelet, and Stochastic Methods. (SIAM, 2005).

116. Timoshenko, J. \& Kuzmin, A. Wavelet data analysis of EXAFS spectra. Computer Physics Communications 180, 920-925 (2009).

117. Baker, C. H., Jordan, D. A. \& Norris, P. M. Application of the wavelet transform to nanoscale thermal transport. Physical Review B 86, (2012).

118. Nadtochenko, V. et al. Ultrafast Spectroscopy of Fano-Like Resonance between Optical Phonon and Excitons in CdSe Quantum Dots: Dependence of Coherent Vibrational WavePacket Dynamics on Pump Fluence. Nanomaterials 7, 371 (2017).

119. Shao, X.-G., Leung, A. K.-M. \& Chau, F.-T. Wavelet: A New Trend in Chemistry. Accounts of Chemical Research 36, 276-283 (2003).

120. Bernardino, A. \& Santos-Victor, J. A Real-Time Gabor Primal Sketch for Visual Attention. in Pattern Recognition and Image Analysis (eds. Marques, J. S., Pérez de la Blanca, N. \& Pina, P.) vol. 3522 335-342 (Springer Berlin Heidelberg, 2005).

121. Field, D. J. Relations between the statistics of natural images and the response properties of cortical cells. Journal of the Optical Society of America A 4, 2379 (1987).

122. Pathak, R. S. \& Dixit, M. M. Continuous and discrete Bessel wavelet transforms. Journal of Computational and Applied Mathematics 160, 241-250 (2003). 
123. Navarro, R. \& Tabernero, A. Gaussian wavelet transform: Two alternative fast implementations for images. Multidim Syst Sign Process 2, 421-436 (1991).

124. Lilly, J. M. \& Olhede, S. C. Generalized Morse Wavelets as a Superfamily of Analytic Wavelets. IEEE Transactions on Signal Processing 60, 6036-6041 (2012).

125. González-Nuevo, J. et al. The Mexican hat wavelet family: application to point-source detection in cosmic microwave background maps. Mon Not R Astron Soc 369, 1603-1610 (2006).

126. Valens, C. A Really Friendly Guide to Wavelets. (1999).

127. Ngui, W. K., Leong, M. S., Hee, L. M. \& Abdelrhman, A. M. Wavelet Analysis: Mother Wavelet Selection Methods. Applied Mechanics and Materials 393, 953-958 (2013).

128. Olhede, S. C. \& Walden, A. T. Generalized Morse wavelets. IEEE Transactions on Signal Processing 50, 2661-2670 (2002).

129. Bennett, B. R., Soref, R. A. \& Alamo, J. A. D. Carrier-induced change in refractive index of InP, GaAs and InGaAsP. IEEE Journal of Quantum Electronics 26, 113-122 (1990).

130. Tzianaki, E. et al. High acoustic strains in Si through ultrafast laser excitation of Ti thin-film transducers. Optics Express 23, 17191 (2015).

131. Dejneka, A. et al. Tensile strain induced changes in the optical spectra of $\mathrm{SrTiO}_{3}$ epitaxial thin films. Phys. Solid State 52, 2082-2089 (2010).

132. Shayduk, R. et al. Direct time-domain sampling of subterahertz coherent acoustic phonon spectra in $\mathrm{SrTiO}_{3}$ using ultrafast x-ray diffraction. Physical Review B 87, (2013).

133. Das, R. R., Yuzyuk, Yu. I., Bhattacharya, P., Gupta, V. \& Katiyar, R. S. Folded acoustic phonons and soft mode dynamics in BaTiO 3 / SrTiO 3 superlattices. Physical Review B 69, (2004).

134. Elzinga, P. A., Lytle, F. E., Jian, Y., King, G. B. \& Laurendeau, N. M. Pump/Probe Spectroscopy by Asynchronous Optical Sampling. Appl. Spectrosc., AS 41, $2-4$ (1987).

135. Cundiff, S. T. \& Ye, J. Colloquium: Femtosecond optical frequency combs. Rev. Mod. Phys. 
$75,325-342$ (2003).

136. Beye, M., Wernet, Ph., Schüßler-Langeheine, C. \& Föhlisch, A. Time resolved resonant inelastic X-ray scattering: A supreme tool to understand dynamics in solids and molecules. Journal of Electron Spectroscopy and Related Phenomena 188, 172-182 (2013). 